\title{
REDUCIBILIDAD EN MATRICES FINITAS NO NEGATIVAS CON APLICACIONES A ECONOMÍA Y CONTROL ESTOCÁSTICO
}

\author{
TESIS QUE PRESENTA: \\ VÍCTOR MANUEL MARTÍNEZ CORTÉS \\ PARA OBTENER EL GRADO DE \\ MAESTRO EN CIENCIAS \\ MATEMÁTICAS APLICADAS E INDUSTRIALES
}

ASESOR: DR. JOSÉ RAÚL MONTES DE OCA MACHORRO

JURADO CALIFICADOR

PRESIDENTE: DR. JUAN GONZÁLEZ HERNÁNDEZ UNAM

SECRETARIO: DR. JULIO CÉSAR GARCÍA CORTE UAM-I

VOCAL:

DR. JOSÉ RAÚL MONTES DE OCA MACHORRO UAM-I 


\section{Índice general}

$\begin{array}{ll}\text { Agradecimientos } & 5\end{array}$

1. Introducción 10

1.1. Descripción General . . . . . . . . . . . . . . . . 10

1.2. Antecedentes . . . . . . . . . . . . . . . . . . 11

1.3. Objetivos. . . . . . . . . . . . . . . . 12

1.4. Estructura del Trabajo . . . . . . . . . . . . . . 13

2. Matrices Finitas no Negativas y sus Potencias 15

2.1. Cadenas de Markov en Espacios Finitos . . . . . . . . . 16

2.2. Propiedades de las Matrices . . . . . . . . . . . . . 25

2.3. Descomposición por Bloques para el Caso: Matrices Irreducibles . . . . . . . . . . . . . . . . . 46

2.4. Descomposición por Bloques para el Caso: Matrices Reducibles . . . . . . . . . . . . . . . . . . 49

3. Convergencia de Métodos Recursivos 60

3.0.1. Convergencia del Caso $\nu=1$ usando Programación Dinámica . . . . . . . . . . . . . . . . 60

3.0.2. Recursión de Programación Dinámica con Matrices no Negativas e Irreducibles . . . . . . . . . . . . . 61 
3.0.3. Recursión con Programación Dinámica de Matrices no Negativas y Reducibles . . . . . . . . . . . . . 64

4. Fundamentos de las Aplicaciones a Control Estocástico 74 4.1. Modelos de Control de Markov . . . . . . . . . . . . . . . . 74

4.2. Políticas . . . . . . . . . . . . . . . 76

4.3. Problema de Control Óptimo . . . . . . . . . . . . . . 77

5. Aplicaciones 79

5.1. Modelo de Leontief . . . . . . . . . . . . . . . . . . . . . . 79

5.2. Caso Neutral al Riesgo: un Juego de Apuestas . . . . . . . . 85

5.2.1. Planteamiento del Problema . . . . . . . . . . . . 86

5.2.2. Caso Algoritmo General . . . . . . . . . . . . . . . 92

5.2.3. Cota de Pronóstico . . . . . . . . . . . . . . . . . 97

5.3. Sensibilidad al Riesgo: una Extensión del Juego de Apuestas 101

5.3.1. Planteamiento del Problema . . . . . . . . . . . . . 101

5.3.2. Algoritmos . . . . . . . . . . . . . . . . 109

6. Conclusiones y Perspectivas 115

6.1. Conclusiones . . . . . . . . . . . . . . . . . . . 115

6.2. Perspectivas . . . . . . . . . . . . . . . 117

7. Apéndices 118

7.1. Un Resultado Auxiliar . . . . . . . . . . . . . . . . 118

7.2. Radio Espectral . . . . . . . . . . . . . . . . . . . 120

7.3. Teorema de Perron Frobenius . . . . . . . . . . . . . . . . . 122

7.4. Sensibilidad al Riesgo . . . . . . . . . . . . . . . . . . 129

7.4.1. Definición y Caracterización de la Aversión al Riesgo 134

7.4.2. Medidas de Aversión . . . . . . . . . . . . . . . . . . 138

7.4.3. Funciones Clásicas de Utilidad . . . . . . . . . . . . 141 
8. Glosario 


\section{Agradecimientos}

Una mención especial que quiero realizar es al Consejo Nacional de Ciencia y Tecnología(CONACyT) por:

(a) El apoyo para realizar mis estudios de Maestría;

(b) El apoyo que se me fue otorgado para complementar mis estudios con una estancia en Praga, República Checa en el período 26/Julio/201328/Septiembre/2013, con una etapa que comprende del 16/Agosto/2013 - 17/Septiembre/2013 en la cual, tuve la fortuna de trabajar con el Dr. Karel Sladký del Institute of Information Theory and Automation (UTIA); y

(c) El apoyo del Proyecto de Cooperación Bilateral Científica y Tecnológica CONACyT-Academy of Sciences of the Czech Republic, número 171396, el cual facilitó el contacto con el Dr. Karel Sladký. 


\section{The Beauty of Life}

There are some chances in life, that give you the opportunity to understand what is really important in the journey. Sometimes we admire people just because they reach something important or because they had something that we dream about it but there are some people that show us in silence the subtlety of life. At this moment I want to say thanks to God for some great teachers that I already had the opportunity to meet.

One of them is Dr. Raúl Montes de Oca who showed me that just a little comment can be transformed into reality and one simple thing can be the next station in life. He offered me the opportunity to reach a goal but not only a goal: he showed me that life is a cycle and today I receive this experience, but in the future I will open the door to somebody else to live his own experience.

The second teacher is Dr. Karel Sladký who offered me the opportunity to open my mind and discover that work is just part of the life, that I need to have passion for Maths, but also have a big heart for the people who support me. Maths is a language and I have discovered that it doesn't matter where I live: the same language can be understood, but also a big ingredient is to have sensibility to live, because this thing play a big role troughout the life. I want to say that Dr Karel's ways of life and his dedication to work really inspired me to give the best of me.

Life is a very big opportunity, you never know exactly what is going to happen but one amazing thing is that you can have the choice to take the risk and discover it or just let it pass without trying. 


\section{Los compañeros del sendero llamado VIDA}

Cuando uno comienza un nuevo proyecto hay muchas cosas que sucederán, nuevas personas llegan, otras simplemente se van y miles de sucesos día con día van aconteciendo.

En este proyecto llamado maestría mi agradecimiento más profundo a Dios por darme la oportunidad de vivir cada uno de los días de mi vida, también por permitirme la bendición de tener unos padres que siempre me han acompañado y me han impulsado en cado paso de mi existencia. También le doy gracias a Dios por conocer a mi maestro espiritual Guru Dev Singh Khalsa el cual me ha ido enseñando lecciones que se han ido convirtiendo en mi guía para este camino.

Una mención especial para mi familia: a mis padres, mi hermana, su esposo, mis sobrinos Celeste y César, a todos mis tíos, a mis primos, mis sobrinos y en general a todos los que siempre han estado ahí de una u otra forma y también para mi linaje, mis abuelos los cuales ya dejaron este planeta; porque día con día me hacen recordar la gran diferencia que existe entre casa y hogar; además de enseñarme lo hermoso que es tener raíces mexicanas.

Cuando llega el momento final de este proyecto, no se puede pensar que las cosas se han obtenido solo, porque cada persona que se ha cruzado aporta un toque especial a la relación. Es en este momento que le quiero agradecer a mis amigos Livtar y Vian por acompañarme en muchas aventuras, compartir conmigo su sabiduría y compañía así como por impulsarme a dar grandes pasos en mi vida y permitirme conocer a Juan Pablo, Kirti, Andrea, Siri Sant, GuruInder, Gurupurkha, Joaquín, Sigrun entre muchos otros, con los cuales he podido compartir grandes experiencias; aquí no se me puede olvidar mi buen amigo Raúl(Hari Sadhana) el cual con sus buenas ideas y alguna que otra locura me enseña que el camino está lleno 
de sorpresas pero que el servicio y el respeto son base fundamental de la vida.

Durante este tiempo que duró la maestría tuve la fortuna de convivir con un gran grupo de compañeros que poco a poco con el pasar del tiempo, algunos dejaron de ser mis compañeros para convertirse en mis amigos, con ellos tuve la oportunidad de vivir muchas experiencias: celebraciones de cumpleaños, congresos, idas al cine, buenas tardes en el cubo y un sin fin de comidas, a cada uno de ellos no me queda más que decirle gracias y que el camino nos guíe a todos a desarrollarnos en nuestros sueños. Les agradezco: Victoria, Liz, Marlene, Juán Luis, Héctor, Adalberto, Marco, Malena, Raquel, Luis Alfonso, Gaby, Daniel y Leticia a cada uno por los momentos compartido y por los que aún están por llegar.

Quiero reconocer a mis amigos-hermanos Rául, Gerardo, Luis, Adrián, Alejandro, Marco y a todos con los que conviví en Sol porque ellos más allá de donde se encuentren me hacen seguir pasando ratos agradables.

La vida es un largo trayecto y muchas veces se comparte con unas personas y luego aparecen nuevas y así se mantiene un constante flujo, este es el momento en donde quiero mencionar a Carol, Elisandro, Elizabeth, Tito, Rocío, Fernanda, Víctor, Francis, Julieta, Silke, Alice, Bertha, Ana por brindarme un gran apoyo durante este tiempo que nos ha tocado compartir y ojalá sea mucho tiempo más.

El tiempo de este período de mi vida está dejando suceder sus últimos minutos y segundos poco a poco se diluye el tiempo para completarse y lo único que me queda decir es gracias a todos los que se cruzaron en mi camino y también a los que no porque al fin del trayecto todos co-existimos en este espacio.

Grandes lecciones me han quedado y muchas otras apenas las estoy integrando, nuevas almas están apareciendo y también otras nos están 
dejando, sin embargo permitir la observación del flujo nos hace recordar que más allá de los eventos y sucesos también tenemos la oportunidad de observar y al mismo tiempo participar en esta hermosa travesía llamada VIDA. 


\section{Capítulo 1}

\section{Introducción}

\subsection{Descripción General}

De manera general, este trabajo se enfoca en las matrices finitas no negativas, sus propiedades, sus potencias y sus aplicaciones. El trabajo está basado en la tesis doctoral del Dr. Zijm [31]. Cabe resaltar que en [31] se trata la propiedad de reducibilidad que a grosso modo explica como se puede diagonalizar, de una manera conveniente, una matriz dada. La reducibilidad dividirá esta tesis en dos grandes grupos: las matrices finitas irreducibles y las matrices reducibles. Más aún, dada esta distinción, aunado a las definiciones de clase básica o clase no básica [31], se trabajará para obtener un tipo de descomposición matricial la cual permitirá analizar el comportamiento de las potencias de las matrices en cuestión.

Posteriormente se estudiará un conjunto de matrices no negativas, el cual puede ser muy grande, pero usando una propiedad a la que se le conocerá con el nombre de "propiedad producto"(pág.4 en [31]), uno se puede restringir a cierto conjunto menor y es sobre éste en el cual se hará un análisis que facilitará la obtención de una matriz que maximiza al conjunto en un sentido apropiado (pág.40 en [31]). 
Una vez teniendo lo anterior se procederá a desarrollar las aplicaciones que materializan la teoría antes presentada. La primera aplicación será en Economía y estará relacionada con la Matriz de Insumo-Producto de Leontief [28]. La segunda aplicación será a los procesos de control de Markov con recompensa total esperada, casos neutral y sensible al riesgo [4], [5], [6], la cual está motivada por un problema de juego de apuestas planteado por Sheldon Ross en su libro "Introduction to Stochastic Dynamic Programming"([21]).

\subsection{Antecedentes}

El estudio sobre la descomposición de las matrices tuvo un gran auge alrededor del año 1870, cuando Camille Jordan en su tratado sobre la sustitución de ecuaciones algebraicas [15] presenta a la comunidad matemática lo que hoy se conoce como la descomposición canónica de Jordan, en donde el resultado principal es la descomposición de un espacio vectorial como suma directa de sus subespacios invariantes. Cabe señalar que Jordan usa una matriz en general, no necesariamente no negativa.

Una vez desarrollado lo anterior, fue entre los años 1960 y 1970 en donde Pease (1965) [20], Mandl y Seneta (1969) [23] y Johnson (1970) [11] entre otros, se adentraron al tema de las matrices no negativas. En estos trabajos se puede observar que hubo un gran desarrollo en la interacción que se tuvo con las cadenas de Markov finitas (Kemeny y Snell [16]), así como en la parte teórica sobre los eigenespacios algebraicos de las matrices no negativas (Rothblum [22]).

Cabe mencionar que la técnica de matrices no negativas que pre- 
sentó el Dr. Zijm [31] en 1983 estaba limitada ya que plantea una gran cantidad de cálculos numéricos que realizarlos a mano, dependiendo del problema planteado, era una tarea casi imposible, sin embargo el Dr. Karel Sladký [24], [25], [26] y [27], continuó trabajando arduamente en esta línea lo que permitió una cooperación para este trabajo. La idea de retomar este trabajo es que actualmente se cuenta con recursos computacionales los cuales permiten la realización de estos cálculos, aunque cabe resaltar que aún existen problemas que con las herramientas de hoy en día, pueden ser computacionalmente muy costosos.

\subsection{Objetivos}

(a) Estudiar las propiedades de las matrices finitas no negativas.

(b) Analizar un ejemplo de la matriz insumo-producto de Leontief.

(c) Resaltar la estructura de las estrategias que se tienen en el problema del jugador planteado por Ross [21], mediante su representación matricial. Ésta permite abordar la selección de la estrategia óptima mediante la maximización de la recompensa en la matriz asociada al problema.

(d) Extender (c) cuando se tiene que el jugador tiene una preferencia al riesgo.

(e) Mostrar que el campo de aplicación de la técnica de matrices no negativas puede ser aplicado en diversos problemas. 


\subsection{Estructura del Trabajo}

El trabajo se encuentra dividido en 6 capítulos:

En el primer capítulo se encuentran las definiciones básicas y una introducción sobre las matrices no negativas, sus resultados más importantes y la estructura que éstas presentan. Para la parte final de este capítulo se dan las caracterizaciones sobre la descomposición que se tiene para los casos irreducible y reducible.

En el segundo capítulo, una vez desarrollada la estructura de las matrices, se procede a enunciar la convergencia cuando se tiene un método recursivo utilizando programación dinámica, ya que lo que se busca es estimar el comportamiento de las potencias de las matrices.

El tercer capítulo presenta una introducción a los procesos de control de Markov, los cuales permitirán la sustentación matemática de la aplicación de los casos neutral y sensible al riesgo.

En el cuarto capítulo se desarrollan las aplicaciones de la técnica de las matrices no negativas. Primero se presentará una matriz de Leontief (de $2 \times 2$ ) la cual ilustra el caso cuando se tiene una recursión del tipo irreducible; cabe señalar que por simplicidad se muestra el caso $2 \times 2$, sin embargo se podría generalizar para dimensión mayor a 2. Posteriormente, en segundo lugar se detallará el ejemplo del jugador propuesto por Ross en la página 76 de [21], en el contexto de los casos neutral y sensible al riesgo. Es importante decir que este capítulo brinda la oportunidad de abordar el

ejemplo del jugador planteado por Ross a través de la estructura matricial, aprovechando las desigualdades de optimalidad correspondientes, asimis- 
mo se adjuntan los programas que permitieron dar la solución númerica de los ejemplos presentados.

El quinto capítulo presenta las conclusiones sobre este trabajo, entre las cuales se presentan algunos problemas que se generan al incrementar el número de estados, las aportaciones y las perspectivas del mismo.

En la parte final de la tesis se tienen las referencias, los apéndices y un glosario de la notación respecto al uso de la letra P. Esta parte está dividida en tres: un resultado sobre la solución de un sistema de ecuaciones, la información sobre el radio espectral y la demostración del Teorema de Perron Frobenius. Este teorema es la base de mucho del trabajo realizado. Por último se presenta una sección sobre "sensibilidad al riesgo", desde su definición, como se mide, y al final una breve descripción de las funciones de utilidad clásicas y su relación al riesgo. 


\section{Capítulo 2}

\section{Matrices Finitas no Negativas y sus Potencias}

Este capítulo se compone de una introducción a la teoría de matrices no negativas, dando referencias sobre algunos resultados importantes de las Cadenas de Markov y se establecen las características de los dos casos que serán el tema central: el caso irreducible y el reducible basados en la propiedad producto; así como el desarrollo de la técnica en la que se hace hincapié sobre la estructura que presenta este tipo de matrices para los dos casos. El final del capítulo permite visualizar la descomposición para los dos casos, lo cual permite abrir una ventana a su aplicación.

Definición 2.0.1. Una matriz $A=\left(a_{i j}\right)$ de tamaño $n \times n$ se dice que es no negativa (positiva) si $a_{i j} \geq 0,\left(a_{i j}>0\right)$. Se denotará por $A \geq 0$ $(A>0)$.

Definición 2.0.2. Se define una matriz de permutación P como aquella que tiene las mismas filas de la matriz identidad, pero no necesariamente en el mismo orden. 
Definición 2.0.3. Sea $n \geq 2$. Una matriz $A$ de orden $n$ se dice reducible si existe una matriz de permutación $P$ tal que:

$$
P^{t} A P=\left(\begin{array}{cc}
A_{11} & A_{12} \\
0 & A_{22}
\end{array}\right) .
$$

donde $A_{11}$ y $A_{22}$ son matrices cuadradas de orden menor que $n$ y $P^{t}$ denota la transpuesta de P. Si no existe tal $P$ entonces se dice que $A$ es irreducible.

Definición 2.0.4. Se define la multiplicidad algebraica de un eigenvalor $\lambda$ de una matriz $A$ de $n \times n$, como el número de veces que aparece repetido como raíz en el polinomio característico.

$(p(t)=\operatorname{det}(A-t I)$ donde det denota el determinante).

i.e máx $\left\{k \in 1, \ldots, n:(t-\lambda)^{k}\right.$ divide a $\left.p(t)\right\}$.

Definición 2.0.5. Se dice que un eigenvalor $\lambda_{i}$ es simple cuando la multiplicidad algebraica es igual a 1 .

Definición 2.0.6. Se define el radio espectral de una matriz cuadrada $P$ como $\sigma(P)=\operatorname{máx}\{|\lambda| \mid \lambda$ es un eigenvalor de $P\}$.

Definición 2.0.7. Sea A una matriz cuadrada, no negativa e irreducible. Sea d el número de valores propios distintos de $A$ de valor absoluto igual al radio espectral $\sigma(A)$. Se dice que $A$ es una matriz primitiva si $d=1$ ó que no es primitiva si $d>1$.

\subsection{Cadenas de Markov en Espacios Finitos}

En esta subsección se presentan algunos resultados relacionados con los tipos de Cadenas de Markov que se pueden tener, la clasificación de los 
estados de éstas, estudiar las clases de comunicación y después crear una clasificación sobre las matrices que emergen de la representación de una Cadena de Markov en su forma matricial.

Considérese una Cadena de Markov con espacio de estados $S$ finito. Como se acostumbra a los elementos de $S$ se les llamará estados.

Definición 2.1.1. Se define la transición de un estado $i$ a un estado $j$ como la probabilidad de ir de $i$ a $j$ en un paso, se denotará por $P_{i j}$.

Definición 2.1.2. Se define la transición en $n$ pasos denotada por $P_{i, j}(n)$ como la probabilidad de ir de $i$ a $j$ exactamente en $n$ pasos i.e. existe $n \in \mathbb{N}$ tal que $P_{i j}(n)$.

\section{Clasificación de Estados:}

- Se dice que un estado $i$ accede al estado $j$ si existe $n \leq 0$ tal que $P_{i j}(n)>0$

- Se dice que los estados $i$ y $j$ se comunican si estos son accesibles entre sí, es decir: $i$ accede a $j$ y $j$ accede a $i$ (denotado por $i \leftrightarrow j$ ). Más aún se sabe que la relación de comunicación es una relación de equivalencia. pág.20, [3]

- Un estado $i$ se dice que es absorbente si una vez llegado a éste ya no se puede salir de él, i.e. $P_{i i}=1$.

- Un estado se dice recurrente si la probabilidad de que se regrese a $i$ dado que se comenzó en $i$ alguna vez es 1, i.e. $P\left(X_{n}=\right.$ $i$ para algún $\left.n \geq 1 \mid X_{0}=i\right)=1$.

- Un estado $i$ es periódico con período "d" (con d un entero positivo) si sólo se puede regresar a él después de $d, 2 d, \ldots, n d$ con $n \in \mathbb{N}$ pasos. $P_{i i}(d)>0$ 
- Un estado se dice aperiódico cuando el período del estado es igual a $1(\mathrm{~d}=1)$.

- Un estado $i$ se dice transitorio si la probabilidad de regresar al estado $i$ dado que se comenzó en el estado $i$ alguna vez es menor que 1 i.e. $P\left(X_{n}=i\right.$ para algún $\left.n \geq 1 \mid X_{0}=i\right)<1$.

Una vez que se tiene lo anterior, el siguiente paso es dar una clasificación respecto a una clase.

Se define una clase de comunicación $C \subset S$ si cualesquiera dos estados de $\mathrm{C}$ se comunican entre sí.

Una clase $\mathbf{C}$ se dice cerrada si ningún estado de $S \backslash C$ puede ser accedido desde un estado de C. Nótese que un estado absorbente es una clase cerrada con un único estado.

Una clase se dice irreducible si es una clase cerrada tal que ninguna subclase de esta es cerrada, lo cual implica que la única clase cerrada es la de todos los estados de la clase.

Lo anterior induce una clasificación más general que es la de cadena.

- Una cadena se dice Irreducible si consta de una única clase de equivalencia bajo la relación de comunicación o equivalentemente el único conjunto cerrado es el de todos los estados.

Nota $\mathrm{Si}$ el espacio de estados es finito, entonces existe al menos un estado recurrente. Veáse pág.54 [3].

- Una cadena se dice Reducible si tiene un subconjunto de estados $C$ desde el cual no es posible alcanzar estados fuera de él. 
- Una cadena se dice Aperiódica si todos sus estados son de período 1.

- Una cadena se dice Regular si es posible ir de un estado a cualquier otro en exactamente $\mathrm{n}$ pasos para alguna $n \in \mathbb{N}$, i.e. existe $n>0$ tal que $P_{i j}(n)>0$ para toda $i, j \in S$.

Nota: Lo anterior explica que la propiedad de ser regular implica que todos los estados se comunican en la etapa n lo cual tiene como consecuencia que la matriz $P^{n}$ es irreducible.

- Una cadena se dice Absorbente si tiene al menos un estado absorbente y desde cualquier estado no absorbente es posible ir a alguno absorbente.

Nota: La matriz de transición de una cadena absorbente siempre se puede llevar a la forma:

$$
P=\left[\begin{array}{cc}
Q & R \\
0 & I
\end{array}\right]
$$

donde la matriz $\mathrm{P}$ es de $(n \times n)$, Q es de $(t \times t)$ y $\mathrm{R}$ es de $(r \times t)$ con $r+t=n$. Aquí I denota la identidad de $(r \times r)$ (Nótese que en I hay r estados absorbentes y estos se pueden reordenar para que aparezcan en las últimas columnas).

Definición 2.1.3. Se define un vector de probabilidad como aquel vector $v \in \Re^{S}$ tal que todas sus entradas son no negativas y la suma de ellas es 1.

Definición 2.1.4. Se define el vector de probabilidades estacionarias(invariante) como el vector que satisface que $v P=v$ 


\section{Cadenas Irreducibles}

\section{Propiedades Generales:}

Si se tiene una matriz $P$ cuyas potencias son $P^{n} \geq 0$ para toda $n$ mayor igual que 1 y tal que $\sigma(P)=1$, entonces se tiene que $P 1=1$, i.e. él $(1,1)$ es un par propio de $P$ (es decir, $P$ tiene el eigenvalor 1 y el eigenvector 1 en donde el eigenvector 1 denota al vector $(1,1, \ldots, 1)$ ).

Nota: Dada una matriz irreducible, por el Teorema de Perron Frobenius [18] pág.536, el 1 es el eigevector de Perron Frobenius asociado al eigenvalor 1. Además no existe otro eigenvector positivo derecho, no negativo; más aún para el mismos eigenvalor, existe un único eigenvector izquierdo $v$ no negativo tal que:

$v^{t} P=v$. Si se normaliza $v$ entonces resulta que:

$v^{t} 1=1$. Entonces, en particular, para una matriz irreducible y primitiva se tiene que:

$$
P=1 v^{t}+\sum_{i>1} \lambda_{i} z_{i} u_{i}^{t}
$$

donde $\lambda_{i}$ representa un eigenvalor de $\mathrm{P}$, donde $u_{i}$ es el eigenvector derecho asociado a $\lambda_{i}$ y $z_{i}$ eigenvector izquierdo. Por lo que:

$$
P^{n}=1 v^{t}+\sum_{i>1} \lambda_{i}^{n} z_{i} u_{i}^{t},
$$

pero como $\left|\lambda_{i}\right|<1 \forall i>1$ por ser primitiva, entonces el 1 es el único eigenvalor sobre el círculo espectral, por lo que se tiene que

$$
\lim _{n \rightarrow \infty} P^{n}=1 v^{t} .
$$

Consecuente se obtiene que si $p^{t}(k)$ denota el k-ésimo paso del vector de probabilidades entonces:

$$
\lim _{k \rightarrow \infty} p^{t}(k)=\lim _{k \rightarrow \infty} p^{t}(0) P^{k}=p^{t}(0) \mathbf{1} v^{t}=v^{t} .
$$


Nótese que lo anterior explica que el vector de probabilidades estacionarias es justamente el vector izquierdo de Perron Frobenius y que es independiente del vector inicial.

Nota: También como una consecuencia del Teorema de Perron Frobenius [18] pág.540, se tiene que dada una matriz P no negativa, irreducible y que no es primitiva de orden $\mathrm{h}>1$ entonces existe una matriz de permutación $V$ tal que la forma canónica de Frobenius $V^{t} P V$ queda de la siguiente manera y además la cadena es periódica con período h:

$$
V^{t} P V=\left[\begin{array}{ccccc}
0 & P_{12} & 0 & \ldots & 0 \\
0 & 0 & P_{23} & \ldots & 0 \\
\vdots & \vdots & \ddots & \ddots & \vdots \\
0 & 0 & \ldots & 0 & P_{h-1, h} \\
P_{h 1} & 0 & \ldots & 0 & 0
\end{array}\right]
$$

\section{Cadenas Reducibles}

Nótese que en una cadena de Markov reducible, es posible mediante operaciones elementales de matrices, llevar la matriz de transición $P$ a la forma:

$$
P^{\prime}:=V^{t} P V=\left[\begin{array}{cc}
X & Y \\
0 & Z
\end{array}\right] .
$$

donde $\mathrm{X}, \mathrm{Y}$ y Z son de dimensiones apropiadas para que la igualdad anterior tenga sentido y $\mathrm{V}$ es una matriz cuadrada de permutación.

En general se puede ver que $P^{\prime}$ es de la siguiente forma: 


$$
P^{\prime}=\left[\begin{array}{ccccccccc}
P_{11} & P_{12} & \ldots & P_{1 r} & \text { । } & P_{1, r+1} & P_{1, r+2} & \ldots & P_{1, m} \\
0 & P_{22} & \ldots & P_{2 r} & \text { । } & P_{2, r+1} & P_{2, r+2} & \ldots & P_{2, m} \\
\vdots & \vdots & \ldots & \vdots & \text { । } & \vdots & \vdots & \ldots & \vdots \\
0 & 0 & \ldots & P_{r r} & \text { । } & P_{r, r+1} & P_{r, r+2} & \ldots & P_{r, m} \\
- & - & - & - & \text { । } & -- & -- & - & - \\
0 & 0 & \ldots & 0 & \text { । } & P_{r+1, r+1} & 0 & \ldots & 0 \\
0 & 0 & \ldots & 0 & \text { । } & 0 & P_{r+2, r+2} & \ldots & 0 \\
\vdots & \vdots & \ldots & \vdots & \text { । } & \vdots & \vdots & \ddots & \vdots \\
0 & 0 & 0 & 0 & \text { । } & 0 & 0 & 0 & P_{m, m}
\end{array}\right]
$$

Donde desde $P_{r+1, r+1}$ hasta $P_{m, m}$ denotan los estados absorbentes, $P_{11}, \ldots, P_{r r}$ y son el complemento de los estados absorbentes, i.e. representan los estados transitorios. Lo anterior se puede denotar como:

$$
P^{\prime}=\left[\begin{array}{cc}
\Gamma_{11} & \Gamma_{12} \\
0 & \Gamma_{22}
\end{array}\right],
$$

donde :

$$
\begin{gathered}
\Gamma_{11}:=\left[\begin{array}{cccc}
P_{11} & P_{12} & \ldots & P_{1 r} \\
0 & P_{22} & \ldots & P_{2 r} \\
\vdots & \vdots & \ldots & \vdots \\
0 & 0 & \ldots & P_{r r}
\end{array}\right], \\
\Gamma_{12}:=\left[\begin{array}{cccc}
P_{1, r+1} & P_{1, r+2} & \ldots & P_{1, m} \\
P_{2, r+1} & P_{2, r+2} & \ldots & P_{2, m} \\
\vdots & \vdots & \ldots & \vdots \\
P_{r, r+1} & P_{r, r+2} & \ldots & P_{r, m}
\end{array}\right],
\end{gathered}
$$




$$
\begin{aligned}
& 0:=\left[\begin{array}{cccc}
0 & 0 & \ldots & 0 \\
0 & 0 & \ldots & 0 \\
\vdots & \vdots & \ldots & \vdots \\
0 & 0 & 0 & 0
\end{array}\right], \mathrm{y} \\
& \Gamma_{22}:=\left[\begin{array}{cccc}
P_{r+1, r+1} & 0 & \cdots & 0 \\
0 & P_{r+2, r+2} & \cdots & 0 \\
\vdots & \vdots & \ddots & \vdots \\
0 & 0 & 0 & P_{m, m}
\end{array}\right] .
\end{aligned}
$$

Cabe observar que el límite, cuando $k \rightarrow \infty$, de $P^{\prime k}$ está dado por:

$$
\lim _{k \rightarrow \infty} P^{\prime k}=\left[\begin{array}{cc}
0 & \left(I-\Gamma_{11}\right)^{-1} \Gamma_{12} L \\
0 & L
\end{array}\right],
$$

siempre y cuando todas las submatrices de $\Gamma_{22}$ sean primitivas.

A continuación se dará un argumento sobre el análisis de la matriz $\lim _{k \rightarrow \infty} P^{\prime k}$ para cada uno de sus bloques.

\section{Para $\Gamma_{11}$}

Como cada $P_{i i}$ es irreducible ó $[0]_{1 \times 1}$ y representa la i-ésima clase transitoria, entonces se tiene que:

$$
\sigma\left(P_{i i}\right)=1 \Rightarrow P_{i i} 1=1
$$


pero $P_{i i} 1 \leq 1$ porque hay bloques $P_{i j}, j \neq i$, no nulos esto implica que: $\sigma\left(P_{i i}\right)<1$, de donde se sigue que:

$$
\sigma\left(\Gamma_{11}\right)<1 \Rightarrow \lim _{k \rightarrow \infty} \frac{I+\Gamma_{11}+\ldots+\Gamma_{11}^{k-1}}{k}=\lim _{k \rightarrow \infty} \Gamma_{11}^{k}=0 .
$$

Las igualdades anteriores se derivan de la Sumabilidad de Césaro aplicada a matrices estocásticas, veáse pág.697 de [19].

\section{Para $\Gamma_{22}$}

Cada $P_{r+j, r+j}$ es irreducible lo que implica que cada $P_{r+j, r+j}$ es simple (i.e., que el conjunto de eigenvalores que coinciden con el radio espectral es igual a 1) y son raíces de la unidad. Por otro lado, los eigenvalores unitarios de $P^{\prime}$ son el conjunto de los eigenvalores unitarios de las submatrices $P_{r+j, r+j}$ y hay que notar que estos pueden aparecer repetidos en más de una submatriz $P_{r+j, r+j}$.

Por lo que se puede aplicar el Teorema de Perron Frobenius (Véase 7.3.6) y tener que:

$v_{j}^{t} P_{j j}=v_{j}^{t}, \quad j=r+1, \ldots, m$. Entonces:

$$
\lim _{k \rightarrow \infty} \frac{I+\Gamma_{22}+\ldots+\Gamma_{22}^{k-1}}{k}=\left[\begin{array}{ccc}
1 v_{r+1}^{t} & 0 & 0 \\
0 & \ddots & 0 \\
0 & 0 & 1 v_{m}^{t}
\end{array}\right] .
$$

Si la matriz anterior se denota por L, se tiene que: $\lim _{k \rightarrow \infty} \Gamma_{22}^{k}=L$. Recuérdese que todas las submatrices de $\Gamma_{22}$ son primitivas.

\section{Para $\Gamma_{12}$}

Por lo hecho para $\Gamma_{11}$ y $\Gamma_{22}$ se tendría que de existir el $\lim _{k \rightarrow \infty} P^{\prime k}$ sería de la 
siguiente forma:

$$
G=\lim _{k \rightarrow \infty} P^{\prime k}=\left[\begin{array}{ll}
0 & Z \\
0 & L
\end{array}\right]
$$

Para analizar $\mathrm{Z}$ recuérdese el hecho de que $R(G)=N(I-P)$ (i.e. Él rango de $G$ es igual al núcleo de $I-P$, pág.633, [19]), debido a que $G$ es el proyector de $N(I-P)$ sobre $R(I-P)$, se obtiene:

$$
(I-P) G=0 \Rightarrow\left[\begin{array}{cc}
I-\Gamma_{11} & -\Gamma_{12} \\
0 & 1-\Gamma_{22}
\end{array}\right]\left[\begin{array}{ll}
0 & Z \\
0 & L
\end{array}\right]=0 \Rightarrow\left(I-\Gamma_{11}\right) Z=\Gamma_{12} L
$$

Como $\left(I-\Gamma_{11}\right)$ no es singular porque $\sigma\left(\Gamma_{11}\right)<1$, entonces se obtiene que: $Z=\left(I-\Gamma_{11}\right)^{-1} \Gamma_{12} L$ siempre y cuando todas las submatrices de $\Gamma_{22}$ sean primitivas.

De donde resumiéndose todo lo anterior se concluye que:

$$
\lim _{k \rightarrow \infty} \frac{I+P^{\prime}+P^{\prime 2}+\ldots+P^{\prime k+1}}{k}=\left[\begin{array}{cc}
0 & \left(I-\Gamma_{11}\right)^{-1} \Gamma_{12} L \\
0 & L
\end{array}\right]
$$

como todas las submatrices de $\Gamma_{22}$ son primitivas entonces se tiene que:

$$
\lim _{k \rightarrow \infty} P^{\prime k}=\left[\begin{array}{cc}
0 & \left(I-\Gamma_{11}\right)^{-1} \Gamma_{12} L \\
0 & L
\end{array}\right]
$$

Para una demostración más detallada del límite de $P^{\prime}$, consúltese págs.697699 de [19].

\subsection{Propiedades de las Matrices}

Definición 2.2.1. Sea $\mathbb{K}$ un conjunto de matrices de tamaño $n \times n, n \in \mathbb{N}$, y sea $P_{i}$ la i-ésima fila de la matriz $P \in \mathbb{K}$. Entonces diremos que $\mathbb{K}$ tiene 
la propiedad producto si para cada subconjunto $V$ de $\{1,2, \ldots, n\}$ y para cada par de matrices $P(1), P(2) \in \mathbb{K}$ lo siguiente se cumple:

La matriz $P(3)$, definida por:

$$
P(3)_{i}:=\left\{\begin{array}{cc}
P(1)_{i} & \text { si } i \in V \\
P(2)_{i} & \text { si } i \in\{1,2, \ldots, n\} \backslash V
\end{array}\right.
$$

es un elemento de $\mathbb{K}$.

\section{Nota (págs.54-55 en [14]):}

(a) Cada conjunto $\mathbb{K}$ que satisface la propiedad producto está dado por una colección de filas admisibles. Es decir, si se tiene dada una colección $\left\{R_{i}, i \in\{1,2, \ldots, n\}\right\}$, de vectores $R_{i}$ no negativos de tamaño $n$, para cada $i \in\{1,2, \ldots, n\}$. Entonces el conjunto $\mathbb{K}$ de todas las matrices tal que su $j$-ésima fila es un elemento de $\left\{R_{i}, i \in\{1,2, \ldots, n\}\right\}$, para cada $j \in\{1,2, \ldots, n\}$, satisface la propiedad producto y viceversa, cada conjunto $\mathbb{K}$ que satisface la propiedad producto está dado por la colección $\left\{R_{i}, i \in\{1,2, \ldots, n\}\right\}$, donde $R_{i}=\left\{A_{i} \mid A \in \mathbb{K}\right\}$, para cada $i \in\{1,2, \ldots, n\}$.

(b) Si se tiene cualquier conjunto finito $K_{0}$ de matrices no negativas, entonces se puede encajar en un conjunto finito $K \supset K_{0}$ el cual cumplirá la propiedad producto. Se puede construir $K$ usando la colección $\left\{R_{i}, i \in\{1,2, \ldots, n\}\right\}$, donde $R_{i}=\left\{A_{i} \mid A \in K_{0}\right\} i \in\{1,2, \ldots, n\}$

. En otras palabras, $K$ es el conjunto de todas las matrices tal que su $j$-ésima fila es igual a la $j$-ésima fila de alguna matriz en $K_{0}$ para cada $i \in\{1,2, \ldots, n\}$.

Ejemplo 1. Sea $\mathbb{K}=\left\{M_{3 \times 3} \mid a_{11}>0\right.$ y $a_{i j} \geq 0$ para $\left.i \neq j\right\}$. Se tiene que $\mathrm{V}$ puede ser de la forma $\{1\},\{1,2\}$ y $\{1,2,3\}$. Para los casos $\{1\}$ y $\{1,2,3\}$ se prueba fácilmente la propiedad producto. Para el caso 
$\{1,2\}$, sean $\mathrm{P}(1)$ y $\mathrm{P}(2)$ dadas por:

$$
P(1):=\left(\begin{array}{lll}
a_{11} & a_{12} & a_{13} \\
a_{21} & a_{22} & a_{23} \\
a_{31} & a_{32} & a_{33}
\end{array}\right), \quad P(2):=\left(\begin{array}{lll}
b_{11} & b_{12} & b_{13} \\
b_{21} & b_{22} & b_{23} \\
b_{31} & b_{32} & b_{33}
\end{array}\right)
$$

entonces dada la definición anterior se obtiene que:

$$
P(3):=\left(\begin{array}{ccc}
a_{11} & a_{12} & a_{13} \\
a_{21} & a_{22} & a_{23} \\
b_{31} & b_{32} & b_{33}
\end{array}\right)
$$

De donde $P(3) \in \mathbb{K}$ debido a que la primera entrada es positiva, por lo que $\mathbb{K}$ tiene la propiedad producto.

Ejemplo 2. Sea $\mathbb{K}=\left\{M_{3 \times 3} \mid 0 \leq a_{i j} \leq 3\right.$ para alguna $i, j$. $\}$, tómese V como $\{1,2\}$ y además

$$
P(1):=\left(\begin{array}{ccc}
0 & 0 & 0 \\
0 & 0 & 0 \\
1 & 2 & 2
\end{array}\right), \quad P(2):=\left(\begin{array}{ccc}
1 & 0 & 0 \\
0 & 0 & 0 \\
0 & 0 & 0
\end{array}\right)
$$

entonces

$$
P(3):=\left(\begin{array}{ccc}
0 & 0 & 0 \\
0 & 0 & 0 \\
0 & 0 & 0
\end{array}\right)
$$

Nótese que $P(3) \notin \mathbb{K}$ por lo que $\mathbb{K}$ no tiene la propiedad producto. 
Proposición 2.2.2. Sea $P$ una matriz cuadrada no negativa y sea $\sigma(P)$ su radio espectral (i.e., $\sigma(P)=\operatorname{máx}\{|\lambda| \mid \lambda$ es un eigenvalor de $P\}$ ). Entonces $\sigma(P)$ es un eigenvalor de $P$ al cual se le puede asociar un eigenvector semipositivo (i.e. es decir el eigenvector puede tener una entrada igual a 0) derecho e izquierdo. Estos eigenvectores son únicos salvo por constantes en el caso que la matriz P sea irreducible, más aún para este caso se pueden elegir estrictamente positivos.

Demostración. Si $P$ es irreducible entonces se tiene que $\sigma(P)$ es simple, por el Teorema 7.3.6 se sigue el resultado.

Por otro lado si $P$ es irreducible con período $d$ entonces existen exactamente $d$ eigenvalores $\lambda_{1}, \lambda_{2}, \ldots, \lambda_{d}$ con $\left|\lambda_{k}\right|=1, k=1, \ldots, d$, así que se puede decir que $\lambda_{k}=\sigma(P) \times \exp (2 \pi k i / d)$ para $k=1, \ldots, d$, de donde todos estos eigenvalores son simples y por el Teorema 7.3.6 se tiene que existe un eigenvector asociado estrictamente positivo. Para el caso en el cual la matriz $P$ es reducible y no negativa, la demostración se encuentran en [10].

\section{Nota:}

(a) Obsérvese que $\sigma(P)>|\lambda|$ para cualquier eigenvalor $\lambda \neq \sigma(P)$.

(b) Considérese una matriz $P$ irreducible y aperiódica. Si se tiene el vector $x(n)=P^{n} x(0), n=1,2, \ldots$, donde $x(0) \geq 0$ es un vector cualquiera de tal manera que la multiplicación tenga sentido, como $P$ es irreducible se puede considerar $u$ el eigenvector derecho estrictamente positivo asociado a $\sigma(P)$ por el Teorema anterior. Si se eligen constantes $c_{1}, c_{2}>0$ tal que el vector $x(0)$ sea acotado de la siguiente forma $c_{1} u \leq x(0) \leq c_{2} u$ entonces para la recursión anterior se puede obtener que: $c_{1}(\sigma(P))^{n} u \leq x(n) \leq c_{2}(\sigma(P))^{n} u, n \in \mathbb{N}$. Esto 
proporciona cotas sobre el comportamiento del vector $x(n)$.

La nota (b) precedente sugiere una pregunta interesante: ¿cuáles matrices no negativas, poseen un eigenvector derecho estrictamente positivo? Parcialmente se sabe que si la matriz es irreducible entonces se tiene una respuesta afirmativa a la pregunta, sin embargo, que una matriz sea irreducible es una condición suficiente para la existencia del eigenvector, pero no necesaria [18]. Con el propósito de responder a la pregunta previa en el caso reducible, a continuación se enunciarán ciertas definiciones y teoremas los cuales permitirán dar respuesta a la pregunta planteada.

Durante esta sección $P$ será una matriz no negativa de tamaño $N \times N$ y se denotará por $S$ al conjunto de estados que está definido por $S:=\{1,2, \ldots, N\}$

Definición 2.2.3. Sea $D$ un subconjunto propio de $S$. La restricción denotada por $P^{D}$ de $P$ a $D \times D$ se le llamará un menor principal de $P$.

Proposición 2.2.4. El radio espectral $\sigma\left(P^{D}\right)$ de cualquier menor principal $P^{D}$ de $P$ no excede el valor del radio espectral $\sigma(P)$ de P. Si $P$ es irreducible, entonces se tiene que $\sigma\left(P^{D}\right)<\sigma(P) \forall P^{D}$; si $P$ es reducible, entonces $\sigma\left(P^{D}\right)=\sigma(P)$ para al menos un menor principal irreducible de $P$.

La demostración de la proposición anterior se puede consultar en la pág.69, [10].

Definición 2.2.5. Una clase de $P$ es un subconjunto $C$ de $S$ tal que la matriz $P^{C}$ es irreducible y es de tal manera que $C$ no puede hacerse más grande sin perder la propiedad de ser irreducible. Se dice que $C$ es una clase básica si $\sigma\left(P^{C}\right)=\sigma(P)$, para cualquier otro caso se dice que $C$ no es básica y en tal caso $\sigma\left(P^{C}\right)<\sigma(P)$. 
Nota: Algo a resaltar es que una matriz $P$ particiona el espacio de estado $S$ en clases básicas $C_{1}, C_{2}, \ldots, C_{n}$; si $P^{(i, j)}$ denota la restricción de $P$ a $C_{i} \times C_{j}, i, j=1, \ldots, n$. Entonces después de un reacomodo en el orden de los estados, a $P$ se le puede reescribir de la siguiente forma [31]:

$$
P:=\left(\begin{array}{cccc}
P^{(1,1)} & P^{(1,2)} & \ldots & P^{(1, n)} \\
& P^{(2,2)} & \ldots & P^{(2, n)} \\
& & \ddots & \vdots \\
& & & P^{(n, n)}
\end{array}\right)
$$

con $P^{(i, j)}=0$ si $i>j$, en la parte inferior de la diagonal aparecen 0. Para realizar el reacomodo de las clases, se utilizará la relación de accesibilidad.

Definición 2.2.6. Se dice que una clase $C_{1}$ accede a la clase $C_{k}$, si un estado $i$ en la clase $C_{1}$ tiene accesibilidad a algún estado $j$ de la clase $C_{k}$. (En el caso de matrices no negativas la accesibilidad de dos estados se interpreta mediante el hecho de que la correspondiente coordenada sea diferente de cero y no necesariamente menor o igual a 1).

Definición 2.2.7. Una clase de $C$ asociada a $P$ se dice que es final, si de $C$ no se puede acceder a otra clase. Una clase de $C$ se dice inicial si ninguna otra clase puede acceder a ésta.

Proposición 2.2.8. La matriz P posee un eigenvector derecho(izquierdo) estrictamente positivo si y solo si sus clases básicas son precisamente la final (inicial).

La demostración de lo anterior se encuentra en [10].

Lema 2.2.9. Sea $P$ una matriz irreducible con radio espectral $\sigma:=\sigma(P)>$ 0 y sea u el eigenvector derecho estrictamente positivo asociado a $\sigma$. Entonces existe una matriz no negativa $P^{*}$ definida por: 


$$
P^{*}:=\lim _{n \rightarrow \infty} \frac{1}{n+1} \sum_{k=0}^{n} \sigma^{-k} P^{k},
$$

y además se tiene que:

$$
P P^{*}=P^{*} P=\sigma P^{*}
$$

y

$$
\left(P^{*}\right)^{2}=P^{*}
$$

Más aún, $p_{i j}^{*}>0$ si y solo si $j$ pertenece a una clase básica de $P$ y además $i$ tiene acceso a $j$ mediante $P$. Si además la restricción de $P$ a cada una de sus clases básicas es aperiódica, entonces se tiene que:

$$
P^{*}=\lim _{n \rightarrow \infty} \sigma^{-n} P^{n} .
$$

Por último, se tiene que la matriz $\left(\sigma I-P+P^{*}\right)$ es no singular.

\section{Demostración:}

Sea $\tilde{P}$ la matriz definida por: $\tilde{P}_{i j}:=\sigma^{-1} u_{i}^{-1} P_{i j} u_{j}, i, j \in S$, la cual existe debido a que $\sigma \neq 0, u \neq 0$ donde $u_{i}$ denota la i-ésima entrada del eigenvector derecho estrictamente positivo y además se tiene que $\tilde{P}$ es estocástica (es decir $\tilde{P}>0$ y $\tilde{P} e=e$ en donde $e$, denota el vector con todas sus componentes iguales a 1) por la forma en que se está definiendo. Como $\tilde{P}$ es irreducible entonces si se define:

$$
P^{*}:=\lim _{n \rightarrow \infty} \frac{1}{n+1} \sum_{k=0}^{n} \sigma^{-k} \tilde{P}^{k},
$$

este límite existe debido a que $\tilde{P}$ es una matriz estocástica e irreducible (págs.691-694 [19]), sin embargo hay que hacer notar que existen dos posibilidades del valor del límite esto debido a si la matriz $\tilde{P}$ es primitiva o 
no es primitiva.

Caso a) $\tilde{P}$ no es primitiva entonces es césaro sumable y entonces:

$$
P^{*}:=\lim _{n \rightarrow \infty} \frac{1}{n+1} \sum_{k=0}^{n} \sigma^{-k} \tilde{P}^{k}
$$

Caso b) $\tilde{P}$ es primitiva y apériodica entonces:

$$
P^{*}:=\lim _{n \rightarrow \infty} \sigma^{-n} \tilde{P}^{n}
$$

Para demostrar que

$$
P P^{*}=P^{*} P=\sigma P^{*}
$$

$\mathrm{y}$

$$
\left(P^{*}\right)^{2}=P^{*}
$$

. La primera parte se sigue directamente de la forma de $P^{*}$, obsérvese lo siguiente:

$$
P P^{*}:=P \lim _{n \rightarrow \infty} \frac{1}{n+1} \sum_{k=0}^{n} \sigma^{-k} \tilde{P}^{k}=\lim _{n \rightarrow \infty} \frac{1}{n+1} \sum_{k=0}^{n} \sigma^{-k} \tilde{P}^{k} P=P^{*} P
$$

ya que $\mathrm{P}$ no depende límite.

Por otro lado como $P$ es irreducible con radio espectral igual a $\sigma$ y eigenvector derecho igual a $u$ entonces se tienen que $P u=\sigma u$, multiplicando por $P *$ se tiene que $P^{*} P u=P^{*} \sigma u=\sigma P^{*} u$.

La demostración de la segunda parte

$$
\left(P^{*}\right)^{2}=P^{*}
$$


se sigue de analizar el límite como sigue a continuación:

$$
\begin{aligned}
\left(P^{*}\right)^{2} & =\left(\lim _{n \rightarrow \infty} \frac{1}{n+1} \sum_{k=0}^{n} \sigma^{-k} \tilde{P}^{k}\right)\left(\lim _{n \rightarrow \infty} \frac{1}{n+1} \sum_{k=0}^{n} \sigma^{-k} \tilde{P}^{k}\right) \\
& =\lim _{n \rightarrow \infty}\left(\lim _{n \rightarrow \infty}\left(\frac{1}{(n+1)^{2}} \sum_{k=0}^{n} \sigma^{-2 k} \tilde{P}^{2 k}\right)\right)=\lim _{n \rightarrow \infty} P^{*} \\
& =P^{*}
\end{aligned}
$$

La demostración de que $p_{i j}^{*}>0$ si y solo si $j$ pertenece a una clase básica de $\mathrm{P}$ y además $i$ tiene acceso a $j$ mediante $P$ se realizará de la siguiente manera. Si suponemos que $p_{i j}^{*}>0$ esto implica por la forma de definición de $P^{*}$ que el estado $i$ tiene acceso a $j$ mediante $P$, más aún como $P^{*}$ es el límite y se sabe que las clases básicas son las que indican el comportamiento al límite entonces $j$ pertenece a una clase básica de $P$. Como todas las afirmaciones anteriores sobre la demostración son si y solo si entonces se tiene que $j$ pertenece a una clase básica de $\mathrm{P}$ y además $i$ tiene acceso a $j$ mediante $P$ implica que $p_{i j}^{*}>0$.

Por último que la matriz $\left(\sigma I-P+P^{*}\right)$ recuérdese que se está asumiendo que $\mathrm{P}$ es apériodica entonces por pág.99 [16] se tiene que $\left(\sigma I-P+P^{*}\right)$ es no singular.

Nota: En [16] se puede encontrar todo un panorama sobre los resultados acerca de las matrices estocásticas. Más aún a la matriz $P^{*}$ se le conoce como el proyector en el espacio nulo de $(\sigma I-P)$ sobre el rango de $\sigma I-P$. Por otro lado a la matriz $\left(\sigma I-P+P^{*}\right)$ se le conoce como la matriz fundamental con respecto a $\mathrm{P}$. Obsérvese que la restricción de $P^{*}$ respecto a cada clase básica de $P$ es estrictamente positiva. Esto ocurre ya que para las clases básicas se tienen matrices irreducibles y como para éstas se tiene la existencia del límite, entonces por la definición de $P^{*}$ ésta sería positiva. Algo muy importante a notar es que en el caso de que la ma- 
triz sea reducible no se tiene la existencia de un eigenvector estrictamente positivo asociado al radio espectral $\sigma$, pero aún en estas circunstancias se puede notar la relevancia de $\sigma$ respecto al comportamiento de la $n$-ésima potencia de la matriz $P$.

Sea $P$ una matriz no negativa. Su norma se tomará como:

$\|P\|:=\operatorname{máx}_{1 \leq i \leq n} \sum_{j=1}^{n} P_{i, j}$.

El vector de puros ceros será denotado por $\underline{0}$.

Lema 2.2.10. Sea $P$ una matriz cuadrada $n \times n$ con radio espectral $\sigma$. Entonces:

a) $\sigma=\lim _{n \rightarrow \infty}\left\|P^{n}\right\|^{1 / n}=\inf _{n}\left\|P^{n}\right\|^{1 / n}$;

b) $\sigma=\sup \{\lambda \mid \exists w>\underline{0}: P w \geq \lambda w\}=\inf \{\lambda \mid \exists w>\underline{0}: P w \leq \lambda w\} ; y$

c) Para cada $\lambda>\sigma$ existe un vector $w>\underline{0}$ tal que $P w \leq \lambda w$.

\section{Demostración:}

La parte a) se encuentra en el apéndice 7.2 y fue tomado de [7]. La parte b) se sigue de a) y para c) tómese a $w=(\lambda I-P)^{-1} e$.

En esta sección lo que es importante destacar es que si $P w<\lambda w$ (componente a componente) esto implica directamente que $P^{n} w<\lambda^{n} w$, si se tiene que $x(0) \leq w$. Esto tiene como resultado que $P^{n} x(0)<\lambda^{n} w$, por lo que se obtendrían cotas para el comportamiento de $P^{n} x(0)$.

Lema 2.2.11. Sea $P$ una matriz irreducible, sea $\sigma$ su radio espectral y sea $x>0$. Si $P x \geq \sigma x$ entonces $P x=\sigma x$. Análogamente, si $P x \leq \sigma x$ entonces $P x=\sigma x$.

\section{Demostración:}

Tómese $P x \geq \sigma x$. Como $P$ es irreducible se tiene que existe el eigenvector 
izquierdo estrictamente positivo asociado a $\sigma$, multiplicando la desigualdad por éste se tendría que $\sigma>\sigma$, contradicción por lo que $P x=\sigma x$. Análogamente, se demuestra la otra desigualdad.

Lema 2.2.12. Sea $P$ una matriz con radio espectral $\sigma$ y supóngase que $P x \geq \lambda x$ para algún número real $\lambda y$ para algún vector $x$ con al menos una entrada positiva. Entonces $\sigma \geq \lambda$.

\section{Demostración:}

Sea $y:=(\lambda I-P) x$, por lo tanto $y \leq 0$. Si $\lambda>\sigma$ entonces se tiene que $\lambda I-P$ no es singular por lo que:

$x=(\lambda I-P)^{-1} y=\sum_{n=0}^{\infty} \lambda^{-(n+1)} P^{n} y \leq 0$, lo que es una contradicción. De donde $\sigma \geq \lambda$.

\section{Descomposición triangular en bloques}

Definición 2.2.13. Una cadena de clases de P es una colección de clases $\left\{C_{1}, C_{2}, \ldots, C_{n}\right\}$ tal que $P_{i_{k} j_{k}}>0$ para algún par de estados $\left(i_{k}, j_{k}\right)$ con $i_{k} \in C_{k}$ y $j_{k} \in C_{k+1}, k=1,2, \ldots, n-1$. Para este caso se dice que la cadena comienza en $C_{1}$ y que termina en $C_{n}$. El tamaño de una cadena se define como el número de clases básicas que ésta contiene.

Definición 2.2.14. Se define la altura (profundidad) de una clase de $C$ de $P$ como el tamaño de la cadena más grande que termina (empieza) en $C$.

Ejemplo 3. Considérese la siguiente matriz:

$$
P:=\left(\begin{array}{lllll}
1 & 4 & 1 & 0 & 0 \\
& 2 & 0 & 0 & 0 \\
& & 1 & 1 & 4 \\
& & & 2 & 4 \\
& & & & 2
\end{array}\right) .
$$


Donde el triángulo inferior de $P$ consiste de puros ceros. En este ejemplo, cada clase de $P$ consiste solamente de un estado; la siguiente gráfica ejemplifica mejor lo que está sucediendo:

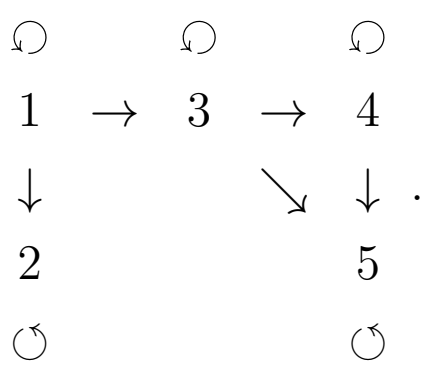

Ahora, para hacer la clasificación de los estados, sólo se tiene que observar que se considera cada clase como un estado, ya que es la única forma de que la clase sea irreducible; más aún, para cada estado se necesita calcular el radio espectral, pero se puede observar que para este ejemplo todo se reduce a calcular el radio espectral respecto a la diagonal es decir $\left(\lambda-P_{i i}\right)$, para todo $i=1, \ldots, 5$ y así se obtiene que las clases básicas son la $\{2\},\{4\},\{5\}$.

\begin{tabular}{|c|c|c|}
\hline & $\begin{array}{c}\text { Clases básicas } \\
\{2\}\{4\}\{5\}\end{array}$ & $\begin{array}{c}\text { Clases no básicas } \\
\{1\},\{3\}\end{array}$ \\
\hline Altura & $1,1,2$ & 0,0 \\
\hline Profundidad & $1,2,1$ & 2,2 \\
\hline
\end{tabular}

Definición 2.2.15. Se define el grado $\nu(P)$ para una matriz $P$, como la longitud de la cadena más larga en $P$.

En el caso del ejemplo anterior se tiene que $\nu(P)=2$.

Dado lo anterior y sin olvidar el objetivo de hacer un reacomodo, las nuevas definiciones como la de altura y la de profundidad muestran 
una idea de que existe una nueva forma de acomodar los estados respecto a estas propiedades. Para poner claridad a lo que se viene vislumbrando se presenta el siguiente lema.

Lema 2.2.16. Sea $\sigma$ el radio espectral de $P$ y $\nu$ su grado. Entonces existe una partición $\{D(\nu), \ldots, D(1), D(0)\}$ del espacio de estados $S$, en donde $D(k)$ representa todas las clases con profundidad $k$, para $k=1, \ldots, \nu$. Si $P^{(k, l)}$ que denota la restricción de $P$ a las clases $D(k) \times D(l)$ entonces $P^{(k, l)}=0$ para $k<l(k, l=0,1, \ldots, \nu)$, una vez ya permutados los estados, la matriz resultante queda de la siguiente forma:

$$
P:=\left(\begin{array}{ccccc}
P^{(\nu, \nu)} & P^{(\nu, \nu-1)} & \ldots & P^{(\nu, 1)} & P^{(\nu, 0)} \\
& P^{(\nu-1, \nu-1)} & \ldots & P^{(\nu-1,1)} & P^{(\nu-1,0)} \\
& & \ddots & \vdots & \vdots \\
& & & P^{(1,1)} & P^{(1,0)} \\
& & & & P^{(0,0)}
\end{array}\right) .
$$

En donde la parte inferior respecto a la diagonal contiene puros ceros. Nótese que para esta descomposición se tiene que $\sigma\left(P^{(k, k)}\right)=\sigma$ para $k=$ $1, \ldots \nu$ y que $\sigma\left(P^{(0,0)}\right)<\sigma$ (si $D(0)$ no es vacío). Más aún, existe un vector $u^{(k)}>\underline{0}$ tal que:

$$
\sigma\left(P^{(k, k)}\right) u^{(k)}=\sigma u^{(k)},
$$

$k=1, \ldots \nu$

\section{Demostración:}

Como el grado de $P$ es $\nu$, entonces existen clases que tienen profundidad $k$ con $k=1, \ldots, \nu$ y podría suceder el caso de que hubiesen clases con profundidad 0 (clases no básicas que no tienen acceso a ninguna clase básica). Se tiene que para cualquier clase que tiene profundidad $k$ ésta no 
tiene acceso a otra clase $l$ con $l>k$, de donde $P^{(k, l)}=0$ para $k<l$. También se obtiene que las clases básicas de profundidad $k$ no tienen acceso a otra clase que tiene la misma profundidad, pero en el caso de que se tenga una clase no básica de profundidad $k$ ésta tiene al menos un acceso a una clase básica con profundidad $k$, por lo que $\sigma\left(P^{(k, k)}\right)=\sigma$ para $k=1, \ldots, \nu$. Por otro lado por la proposición 2.2.4 y las definiciones de clase básica y no básica se tiene que $\sigma\left(P^{(0,0)}\right)<\sigma$, por la proposición 2.2.8 se tiene la existencia de los vectores $u^{(k)}>\underline{0}$, de donde por lo anterior se cumple que:

$$
\sigma\left(P^{(k, k)}\right) u^{(k)}=\sigma u^{(k)},
$$

$k=1, \ldots \nu$

Definición 2.2.17. La partición $(D(\nu), D(\nu-1), \ldots, D(1), D(0))$ en la que $D(k)$ contiene todas las clases con profundidad $k(k=\nu, \nu-1, \ldots, 1,0)$ se le conoce como la partición principal de $S$ con respecto a $P$.

Si se vuelve a utilizar el ejemplo 3 y se toma la respectiva partición principal, entonces resulta que:

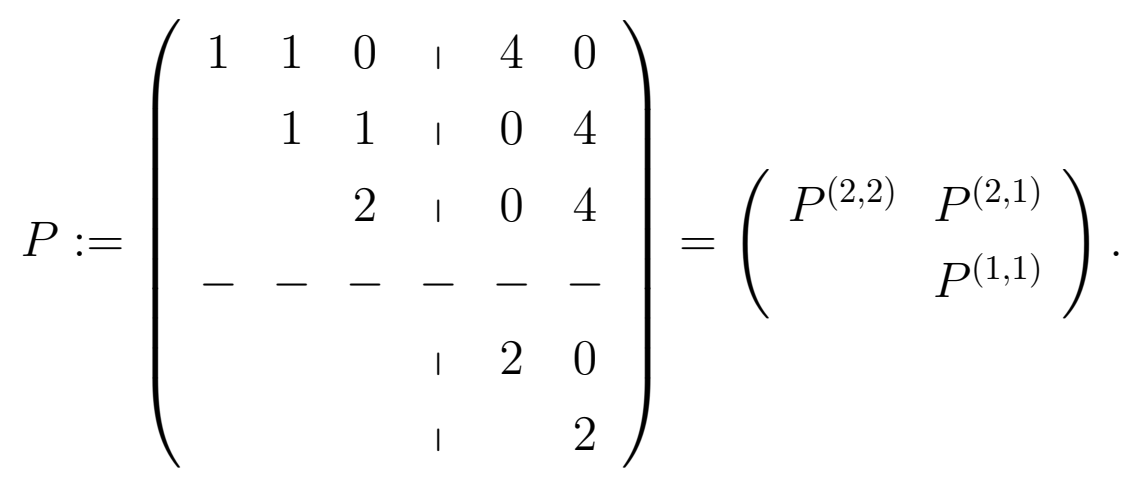

Además se tiene que tanto $P^{(2,2)}$ como $P^{(1,1)}$ tienen eigenvectores estrictamente positivos, asociados al eigenvalor 2. Esto indica que el comportamiento al límite depende en gran medida de las clases básicas y de la 
posición en donde se encuentran éstas.

Lema 2.2.18. Sea $P$ una matriz con radio espectral $\sigma$, grado igual a $\nu$ y sea $\{D(\nu), \ldots, D(1), D(0)\}$ la partición principal de $S$ con respecto a $P$. Elíjase $x(0)>\underline{0}$, y sea $x(n)=P^{n} x(0), n=1,2, \ldots$ Entonces existen constantes $c_{1}, c_{2}$ y vectores $u^{(k)}>\underline{0}$ tales que satisfacen:

$$
\sigma\left(P^{(k, k)}\right) u^{(k)}=\sigma u^{(k)},
$$

$k=1, \ldots \nu, y$ además se tiene que:

$$
c_{1} u_{i}^{(k)} \leq\left(\begin{array}{l}
n \\
k-1
\end{array}\right)^{-1} \sigma^{-n} x(n)_{i} \leq c_{2} u_{i}^{(k)}
$$

$i \in D(k) ; k=1, \ldots, \nu$ y que:

$$
\lim _{n \rightarrow \infty} \sigma^{-n} x(n)_{i}=0
$$

$i \in D(0)$

La demostración de este lema se encuentra en [29].

Nota: El lema anterior, muestra una relación entre el comportamiento del vector $x(n)=P^{n} x(0), n=0,1,2, \ldots$ y la posición en las que se encuentran las clases básicas de $P$.

Como se puede notar el concepto de profundidad juega un papel muy importante en el análisis de la descomposición matricial que se está haciendo. Análogamente se puede hacer lo mismo pero usando el concepto de altura con respecto a $x(0)^{t} P^{n}$; sin embargo, para este trabajo se usará la profundidad.

En la parte anterior del trabajo se tomó que $D(0)=\emptyset$ (veáse el enunciado del Lema 2.1.19), sin embargo habrá ocasiones en las que esto no sea cierto, así que se dará una forma más general para cuando $D(0) \neq \emptyset$ y entones 
$\sigma\left(P^{(0,0)}\right) \neq 0$

Lema 2.2.19. Sea $P$ una matriz cuadrada y no negativa. Entonces existe un entero $r=r(P)$ y una partición $\{I(1), I(2), \ldots I(r)\}$ del espacio de estado $S$, tal que las siguientes propiedades se cumplen:

(a) Sea $P^{(k, l)}$ que denota la restricción de $P$ a $I(k) \times I(l)$. Entonces $P^{(k, l)}=\underline{0}$ si $k>l, k, l=1, \ldots, r$.

(b) Para $k \leq l$, se tiene que $\sigma\left(P^{(k, k)}\right) \geq \sigma\left(P^{(l, l)}\right)$, con igualdad solamente, cuando para cada estado de $I(k)$ se tiene acceso a algún estado de $I(l), k, l=1, \ldots r$.

(c) Existe un vector estrictamente positivo $u^{(k)}$ tal que:

$$
P^{(k, k)} u^{(y)}=\sigma\left(P^{(k, k)}\right) u^{(k)}
$$

$k=1, \ldots, r$.

(d) Se elije $x(0)>\underline{0}$ y sea $x(n)=P^{n} x(0)$ para $n=1,2, \ldots$ Para cada $k \in\{1,2, \ldots r\}$ se define el entero $t_{k}$ como:

$$
t_{k}:=\min \left\{l \mid 0<l \leq r-k, \sigma\left(P^{(k+l, k+l)}\right)<\sigma\left(P^{(k, k)}\right)\right\}
$$

En caso de que no exista el minimo entonces se define $t_{k}:=r-k+1$. Más aún, si $\sigma\left(P^{(r, r)}\right) \neq 0$, entonces existen constantes positivas $c_{1}, c_{2}$ que solo depende de $x(0)$ tales que:

$$
c_{1} u_{i}^{(k)} \leq\left(\begin{array}{l}
n \\
t_{k}^{-1}
\end{array}\right)^{-1} \sigma\left(P^{(k, k)}\right)^{-n} x(n)_{i} \leq c_{2} u_{i}^{(k)},
$$

para $i \in I(k), k=1, \ldots, r$ y $n \in \mathbb{N}$. 
La demostración de este lema se sigue del Lema 2.2.18 sólo notando que para cuando $P^{(0,0)} \neq \emptyset$ se vuelve a realizar el proceso de descomposición.

Definición 2.2.20. A la partición $\{I(1), I(2), \ldots, I(r)\}$ de la parte anterior se le conoce como la partición espectral de $S$ con respecto a $P$.

Nota: Es importante observar que la parte anterior permite resolver problemas en el que la multiplicidad algebraica coincide con la multiplicidad geométrica, pero ¿qué sucede en el caso en el que esto no ocurre?. Como se puede empezar a vislumbrar los procedimientos que se están usando tienen una intrínseca relación con los conocimientos que se tienen en el Álgebra Lineal sobre los eigevectores y los eigenvectores generalizados. En esta parte, el enfoque será adentrarnos en el caso en el que las multiplicidades no coinciden.

Definición 2.2.21. Sea $P$ una matriz cuadrada no negativa, con radio espectral $\sigma$ y para cada $k \in \mathbb{N}$, sea $\mathbb{N}^{k}(P)$ el espacio nulo de $(P-\sigma I)^{k}$. El indice $\eta(P)$ de $P$ se define como el entero no negativo más pequeño para el cual se cumple que $N^{k}(P)=N^{k+1}(P)$.

Si $P$ es una matriz de $N \times N$ con radio espectral $\sigma$ y con índice $\eta$, entonces se tiene que $\eta \leq N$ y además:

$$
N^{1}(P) \varsubsetneqq N^{2}(P) \varsubsetneqq \cdots \varsubsetneqq N^{\eta}(P)=N^{k}(P),
$$

para $k \geq \eta$.

Los elementos de $N^{\eta}(P)$ son llamados los eigenvectores generalizados. Si $x \in N^{k}(P) \backslash N^{k-1}(P)$ se le conoce como un eigenvector generalizado de orden $k$. 
Antes de continuar con lo que sigue, obsérvese lo siguiente:

Ejemplo 4. Tómese $P$ como:

$$
P:=\left(\begin{array}{ll}
2 & 4 \\
0 & 2
\end{array}\right), w(1)=\left(\begin{array}{l}
4 \\
0
\end{array}\right), w(2)=\left(\begin{array}{l}
1 \\
1
\end{array}\right) .
$$

Un hecho a resaltar es que:

$$
P w(2)=2 w(2)+w(1), \quad P w(1)=2 w(1) .
$$

En donde $w(2)$ es un eigenvector generalizado de orden 2 y lo que sobresale es que $w(2)$ es estrictamente positivo.

Teorema 2.2.22. Sea $P$ una matriz cuadrada, no negativa con radio espectral $\sigma$ y con grado $\nu$. Entonces para $k=1, \ldots, \nu$ existen eigenvectores generalizados $w(k)$ de orden $k$ tales que:

$$
\begin{gathered}
P w(v)=\sigma w(v) \\
P w(k)=\sigma w(k)+w(k+1)
\end{gathered}
$$

$k=1, \ldots, \nu-1$. Sea $\{D(\nu), D(\nu-1), \ldots, D(1), D(0)\}$ la partición principal de $S$ con respecto a $P$. Entonces los vectores $w(k)$ pueden ser elegidos de tal manera que, para $k=1, \ldots, \nu$ :

$$
w(k)_{i}>0
$$

$i \in \bigcup_{l=k}^{\nu} D(l), y$

$$
w(k)_{i}=0
$$

para $i \in \bigcup_{l=0}^{k-1} D(l)$ 


\section{Demostración:}

Considérese $P^{(k, l)}$ la restricción de $P$ a las clases $D(k) \times D(l)$, y para $k=1,2, \ldots, \nu$ defínase:

$$
R(k):=\left(\begin{array}{ccccc}
P^{(k, k)} & P^{(k, k-1)} & \ldots & P^{(k, 1)} & P^{(k, 0)} \\
& P^{(k-1, k-1)} & \ldots & P^{(k-1,1)} & P^{(k-1,0)} \\
& & \ddots & \vdots & \vdots \\
& & & P^{(1,1)} & P^{(1,0)} \\
& & & & P^{(0,0)}
\end{array}\right) .
$$

Obsérvese que $R(\nu)=P$. Se demostrará por inducción sobre $k$ que para $k=1, \ldots, \nu$ existe una sucesión de eigenvectores generalizados $y(1), y(2), \ldots, y(k)$ tales que:

$$
\left\{\begin{array}{l}
R(k) y(l)=\sigma y(l)+y(l+1), \quad l=1, \ldots, k-1 \\
R(k) y(k)=\sigma y(k)
\end{array}\right.
$$

con $y(l)_{i}=0$ para $i \in \bigcup_{n=0}^{l-1} D(n), y(l)_{i}>0$ para $i \in \bigcup_{n=l}^{k} D(n)$.

Por el lema 2.2.16 existe un vector $y(1)$ definido en $D(0) \cup D(1)$ tal que:

$$
R(1) y(1)=\sigma y(1)
$$

en donde $y(1)_{i}=0$, para $i \in D(0)$ y $y(1)_{i}>0$, para $i \in D(1)$.

Supóngase que existen vectores $x(1), x(2), \ldots x(k)$ definidos en $\bigcup_{n=0}^{k-1} D(n)$, tales que:

$$
\begin{aligned}
& R(k-1) x(l)=\sigma x(l)+x(l+1) \\
& l=1, \ldots, k-1, \text { con } x(l)_{i}=0, \text { para } i \in \bigcup_{n=1}^{l-1} D(n) \text { y } x(l)_{i}>0 \text { para } \\
& i \in \bigcup_{n=l}^{k-1} D(n)(l=1, \ldots, k) .
\end{aligned}
$$


Lo que falta, es encontrar vectores $y(1), y(2), \ldots y(k)$ definidos en $\bigcup_{n=0}^{k} D(n)$ tal que (2.3) se cumpla. Se puede pensar en definir:

$$
y(l)_{i}:=x(l)_{i} \text { para } i \in \bigcup_{n=0}^{k-1} D(n)
$$

$l=1, \ldots k$. Nótese que $x(k)=\underline{0}$. Si $x(n)^{l}$ denota la restricción de $x(n)$ a $D(l)$ para $n=1, \ldots, k$ y $l=0,1, \ldots, k-1$ y $y(n)^{k}$ denota la restricción de $y(n)$ a $D(k)$ para $n=1, \ldots, k$, entonces $(2.3)$ se reduce a:

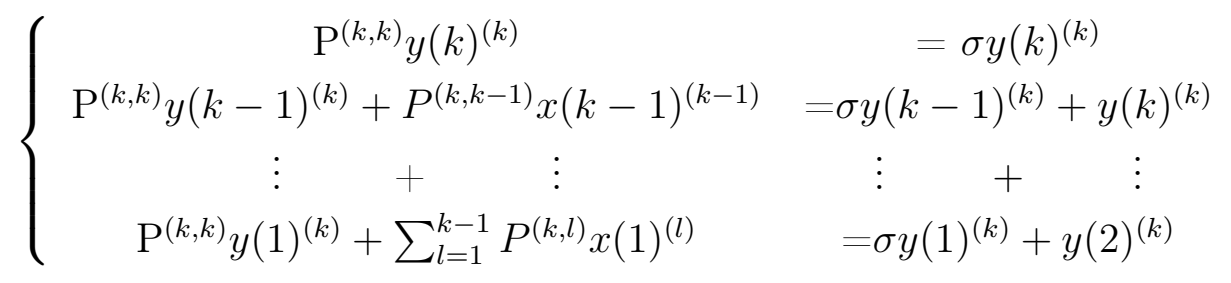

Obsérvese que $P^{(k, k)}$ posee un eigenvector estrictamente positivo asociado $\sigma$. Por el Lema 7.1.1 existe solución al sistema (2.4), llamémosle $\left\{y(k)^{(k)}, \ldots, y(1)^{(k)}\right\}$ de tal manera que:

$$
y(k)^{(k)}=\left(P^{(k, k)}\right)^{*} P^{(k, k-1)} x(k-1)^{(k-1)} .
$$

Como la restricción de $\left(P^{(k, k)}\right)^{*}$ a las clases básicas de $P^{(k, k)}$ es estrictamente positiva, esto por el Lema 1.0 .8 y como cada estado en $D(k)$ tiene acceso a algún estado en $D(k-1)$, se sigue que $x(k-1)^{k-1}>\underline{0}$, por lo tanto $y(k)^{(k)}>0$.

Dado esto, se ha encontrado una solución $\{y(1), \ldots y(k)\}$ de $(2.3)$. Si se definen $\{w(1), \ldots w(k)\}$ como:

$$
\left\{\begin{array}{l}
\mathrm{w}(\mathrm{k})=\mathrm{y}(\mathrm{k}) \\
\mathrm{w}(\mathrm{l})=\mathrm{y}(\mathrm{l})+\alpha w(l+1) \quad l=k-1, k-2, \ldots, 1 ; \alpha \in \mathbb{R}
\end{array}\right.
$$

éstos también cumplen con $(2.3)$ debido a que $\{y(1), \ldots y(k)\}$ la satisfacen. Como $y(k)^{(k)}>\underline{0}$ entonces se puede elegir $\alpha$ lo suficientemente grande, de tal manera que $w(l)_{i}>0$, para $i \in D(k), l=1,2, \ldots k$. y con esto se tiene el resultado para $R(k)$. 
Corolario 2.2.23. Sea $P$ una matriz cuadrada con radio espectral $\sigma$, indice $\nu$ y supóngase que cada clase no básica de $P$ tiene acceso a alguna clase básica de P. Entonces debe de existir un eigenvector $w(\nu)$ de orden $\nu$ asociado a $\sigma$, tal que $w(\nu)>\underline{0}$.

La demostración se sigue directamente del Teorema 2.2.22 tomando $R=R(\nu)$. Para este caso se tiene que existe $w(\nu)=y(\nu)$ y además se tiene que $y(\nu)>\underline{0}$.

El Teorema 2.2.22 exhibe una relación muy importante entre las clases básicas y las no básicas de $P$, además de que ofrece una idea sobre el comportamiento de las potencias.

Teorema 2.2.24. Sea $P$ una matriz cuadrada con radio espectral $\sigma$ y grado $\nu$. Sea $\{D(\nu), D(\nu-1), \ldots, D(1), D(0)\}$ la partición principal de la matriz $P$ y sean $\{w(\nu), w(\nu-1), \ldots, w(1)\}$ definidos como en el Teorema 2.2.22. Elíjase $x(0)>\underline{0}$ y sea $x(n):=P^{n} x(0), n=1,2, \ldots$, Entonces deben existir constantes $c_{1}$ y $c_{2}$ tales que:

$$
c_{1} \sum_{k=1}^{\nu}\left(\begin{array}{l}
n \\
k-1
\end{array}\right) \sigma^{n-k-1} w(k)_{i} \leq x(n)_{i} \leq c_{2} \sum_{k=1}^{\nu}\left(\begin{array}{l}
n \\
k-1
\end{array}\right) \sigma^{n-k-1} w(k)_{i}
$$

$i \in S \backslash D(0), n \in \mathbb{N}$ y además,

$$
\lim _{n \rightarrow \infty} \sigma^{-n} x(n)_{i}=\underline{0}
$$

$i \in D(0)$.

\section{Demostración:}

Tómese $P^{(k, l)}$ la restricción de $P$ a $D(k) \times D(l)$ y sea $w(k)^{(l)}$ la restricción de $w(k)$ a $D(l)$, para todo $k, l$. Se sabe que $P^{(k, l)}=0$ si $k<l$ y entonces $w(k)^{(l)}=\underline{0}$ para $k>l$. Como se tiene que $\sigma\left(P^{(0,0)}\right)<\sigma$, entonces por 
el Lema 2.2.10 (c), existe un número real no negativo $\lambda<\sigma$ y un vector $w(\lambda)>\underline{0}$ el cual está definido en $D(0)$, que cumple:

$$
P^{(0,0)} w(\lambda) \leq \lambda w(\lambda) .
$$

Dado lo anterior, se puede elegir $w(\lambda)$ tal que $P^{(k, 0)} w(\lambda) \leq w(k)^{(k)}$, para $k=1, \ldots, \nu$, así mismo se puede elegir una constate $c>0$ de tal manera que $x(0)_{i} \leq c w(1)_{i}$ para $i \in S \backslash D(0)$ y $x(0)_{i} \leq c w(\lambda)_{i}$, para $i \in D(0)$. Ahora si se utiliza el procedimiento de inducción, se puede mostrar fácilmente que:

$$
x(n)_{i} \leq c \lambda^{n} w(\lambda)_{i}
$$

$i \in D(0), \mathrm{y}$

$$
x(n)_{i} \leq c\left\{\sum_{k=1}^{\nu} \sigma^{n-k+1} w(k)_{i}+\sigma^{n-1}\left(1-\lambda \sigma^{-1}\right)^{-1} w(l)_{i}\right\},
$$

$i \in D(l), l=1, \ldots, \nu$.

El otro lado de la desigualdad se prueba de manera análoga, poniendo atención en la elección de la constante.

\subsection{Descomposición por Bloques para el Ca- so: Matrices Irreducibles}

Como por el Teorema de Perron Frobenius 7.3.6 una matriz P no negativa e irreducible posee un eigenvector derecho $u$ estrictamente positivo asociado con el radio espectral $\sigma$. Entonces automáticamente es posible establecer cotas para el vector $x(n)$ definido por:

$$
x(n)=P x(n-1),
$$


$n \in \mathbb{N}, x(0)>0$. Lo cual ya se había comentado en la pág.25 nota (b) de este trabajo; debido a que se tiene:

$$
c_{1} \sigma^{n} u \leq x(n) \leq c_{2} \sigma^{n} u,
$$

$n \in \mathbb{N}$ en donde se escogen constantes $c_{1}, c_{2}$ de tal forma que:

$$
c_{1} u \leq x(0) \leq c_{2} u .
$$

Ahora lo interesante es contestar a la siguiente pregunta ¿cuándo se puede decir algo similar para la recursión que se presenta a continuación?:

$$
x(n)=\operatorname{máx}_{P \in \mathbb{K}} P x(n-1),
$$

$n \in \mathbb{N}, x(0)>0$, dado que se supone que el conjunto restricción $\mathbb{K}$ consiste sólo de matrices irreducibles.

Lema 2.3.1. Sea $\mathbb{K}$ formado únicamente de matrices irreducibles y sea $\hat{\sigma}:=\operatorname{máx}\{\sigma(P) \mid P \in \mathbb{K}\}$. Entonces existe una matriz $\hat{P} \in \mathbb{K}$ con radio espectral $\hat{\sigma}$ con su respectivo eigenvector derecho u estrictamente positivo tal que:

$$
\hat{P} \hat{u}=\operatorname{máx}_{P \in \mathbb{K}} P \hat{u}=\hat{\sigma} \hat{u} .
$$

\section{Demostración:}

Considérese $P(0) \in \mathbb{K}$ arbitraria pero fija.

Para $m=0,1,2, \ldots$, escójase $P(m+1) \in \mathbb{K}$ tal que:

$$
P(m+1) u(m)=\operatorname{máx}_{P \in \mathbb{K}} P u(m),
$$

con

$$
P(m+1)_{i}:=P(m)_{i} \text { si }(P(m) u(m))_{i}=\left(\operatorname{máx}_{P \in \mathbb{K}} P u(m)\right)_{i} .
$$

En donde $u(m)$ denota el eigenvector derecho estrictamente positivo asociado al radio espectral $\sigma_{m}$ de $P(m)$. 
Deténgase si $P(m+1)=P(m)$ y entonces defínase $\hat{\sigma}:=\sigma(m), \hat{u}:=u(m)$ y $\hat{P}=P(m)$. En caso contrario continúe con el procedimiento.

Por la forma de esta construcción se tiene que:

$$
P(m+1) u(m) \geq P(m) u(m)=\sigma_{m} u(m) .
$$

Por lo que implica el radio espectral, si se tiene que $\sigma_{m+1}=\sigma_{m}$ esto tiene como consecuencia que $P(m+1) u(m)=\sigma_{m} u(m)=P(m) u(m)$, con lo que se finalizaría el proceso. Por otro lado, si se tiene el caso de que $P(m+1) \neq P(m)$ se sigue entonces que $\sigma_{m+1}>\sigma_{m}$, lo que significa que el proceso no se cicla, por lo que, el procedimiento termina después de un número finito de pasos; esto debido a que $\mathbb{K}$ es finito.

Del Lema 2.3.1 se tiene que:

$$
x(n)=\operatorname{máx}_{P \in \mathbb{K}} P x(n-1),
$$

$n \in \mathbb{N} x(0)>\underline{0}$, y con esto se obtiene que:

$$
c_{1} \hat{\sigma}^{n} \hat{u} \leq x(n) \leq c_{2} \hat{\sigma}^{n} \hat{u}
$$

$n \in \mathbb{N}$, siempre y cuando la elección de las constantes $c_{1}$ y $c_{2}$ cumplan con que: $c_{1} \hat{u} \leq x(0) \leq c_{2} \hat{u}$.

Lo precedente nos permite acotar el vector $x(n)$ y decir qué sucede cuando $n \rightarrow \infty$.

Nota: Al utilizar matrices irreducibles, por el Teorema 7.3.6 se tiene un eigenvector $u$ asociado al radio espectral $\sigma$, lo que implica que la partición inducida es la dada por el Lema 2.2.19. 


\subsection{Descomposición por Bloques para el Ca- so: Matrices Reducibles}

Teorema 2.4.1. Sea $\mathbb{K}$ un conjunto finito de matrices no negativas con la propiedad producto. Entonces existe un entero $r$ y una partición $\{I(1), \ldots, I(R)\}$ del espacio de estados $S$, tal que las siguientes propiedades se cumplen:

(a) Sea $P^{(k, l)}$ la cual denota la restricción a $I(k) \times I(l)$.

entonces $P^{(k, l)}=0$ si $k>l(k, l=1, \ldots, r)$ para cada $P \in \mathbb{K}$.

(b) Existe una matriz $\hat{P} \in \mathbb{K}$ y vectores estrictamente positivos $\hat{u}^{(k)}$ definidos sobre $I(k)$ tal que:

$$
\hat{P}^{(k, k)} \hat{u}^{(k)}=\operatorname{máx}_{P \in \mathbb{K}} P^{(k, k)} \hat{u}^{(k)}=\hat{\sigma}_{k} \hat{u}^{(k)},
$$

$k=1,2, \ldots, r$ en donde $\hat{\sigma}_{k}:=\sigma\left(\hat{P}^{(k, k)}\right), \quad k=1,2, \ldots, r$.

Para $k \leq l$ en donde se tiene $\hat{\sigma}_{k} \geq \hat{\sigma}_{l}$ con igualdad si cada estado en $I(k)$ tiene acceso a algún estado en $I(l)$ bajo $\hat{P}$.

(c) Sea $x(0)>\underline{0}$ y sea $x(n)$ definido como en 2.7; Para cada $k \in$ $\{1,2, \ldots, r\}$ sea $t_{k}$ un entero positivo definido de la siguiente forma:

$t_{k}:= \begin{cases}\min \left\{j \mid j>0, \hat{\sigma}_{k+j}<\sigma_{k}\right\} & \text { si existe } j ; \\ r-k+1 & \text { en cualquier otro caso. }\end{cases}$

Entonces deben existir constantes $c_{1}$ y $c_{2}$ positivas de tal forma que:

$$
c_{1} u_{i}^{k} \leq\left(\begin{array}{c}
n \\
t_{k}-1
\end{array}\right)^{-1} \hat{\sigma}_{k}^{-n} x(n)_{i} \leq c_{2} u_{i}^{(k)}
$$

para cada $i \in I(k), k=1,2, \ldots, r$ y $n \in \mathbb{N}$.

La demostración de este teorema se hará mediante la construcción de un método iterativo, el cual permitirá darle solución al problema de las 
matrices reducibles.

\section{Demostración del Teorema 2.4.1.}

Para la primera parte se necesita:

\section{Procedimiento de Completación:}

Comenzar: Sea $P(0) \in \mathbb{K}$ dada.

Para $m=0,1,2, \ldots$, aplíquese el siguiente paso iterativo hasta que la condición se satisfaga.

\section{Paso iterativo:}

Mediante la permutación de los estados se puede escribir $P(m)$ (el resultado de la $m$-ésima iteración) en la forma triangular del Lema 2.2.19. Sea $\left\{I_{1}, \ldots, I_{r_{m}}\right\}$ la partición espectral de $S$ con respecto a $P(m)$. Para esto hay dos posibilidades:

(a) Si para $k=1,2, \ldots, r_{m-1}$ y para toda $P \in \mathbb{K}$, $P_{i j}=0$, para toda $i \in \bigcup_{l=k+1}^{r_{m}} I_{l}(m)$ y para toda $j \in I_{k}(m)$ entonces definase $P(m+1):=P(m)$.

(b) Si, por el contrario existe un entero $t, 1 \leq t \leq r_{m}$, tal que para $1 \leq k<t$ y para toda $P \in \mathbb{K}$

$$
P_{i j}=0,
$$

para todo $i \in \bigcup_{l=k+1}^{r_{m}} I_{l}(m)$ y para toda $j \in I_{k}(m)$, mientras que para $k=t$ existe $P \in \mathbb{K}$ de tal forma que:

$$
P_{i j}>0
$$

para algún $i \in \bigcup_{l=k+1}^{r_{m}} I_{l}(m)$ y alguna $j \in I_{k}(m)$,

entonces el siguiente paso a realizarse es: 
Para $P \in \mathbb{K}$, sea $D(m, P)$ que denota el conjunto de estados que tienen acceso a $I_{k}(m)$ bajo $P$ y sea $D(m):=\bigcup_{P \in \mathbb{K}} D(m, P)$.

Como $\mathbb{K}$ tiene la propiedad producto, entonces debe existir una matriz $\tilde{P}$ de tal forma que todos los estados en $D(m) \backslash I_{t}(m)$ tienen acceso a $I_{t}(m)$ mediante $\tilde{P}$. Ahora, determínese $P(m+1)$ tal que:

$P(m+1)_{i}:= \begin{cases}\tilde{P}_{i} & \text { si } i \in D(m) \backslash I_{t}(m) \\ P(m)_{i} & \text { en cualquier otro caso }\end{cases}$

Pare si $P(m+1)=P(m)$.

Defínase $\bar{P}(0):=P(m)$.

A $\bar{P}(0)$ se le conoce como la completación de $P(0)$.

Para probar que el procedimiento de completación no se cicla y que termina después de un número finito de pasos se necesita probar el siguiente resultado.

Definición 2.4.2. Sean $\left(y_{1}, y(2), \ldots, y(n)\right)$ y $\left(z_{1}, z_{2}, \ldots, z(n)\right)$ dos secuencias de vectores con entradas en los reales. Se dice que $\left(y_{1}, y(2), \ldots, y(n)\right) \succ$ $\left(z_{1}, z_{2}, \ldots, z(n)\right)$ si $y_{1}>z_{1}$ ó si para alguna $k \in 1, \ldots n$ se tiene que $y_{l}=z=l$ para $l=1,2, \ldots k$ y $y_{k+1}>z_{k+1}$. Las definiciones para $\prec, \preceq$, $\succeq$ se siguen de la anterior. A este orden se le conoce con el nombre de Lexicográfico.

Lema 2.4.3. Sea $P(m)$ la matriz que resulta de la m-ésima iteración del proceso de completación $(m=0,1, \ldots)$, entonces

$$
\left(\sigma_{i}(P(m+1)), \nu_{i}(P(m+1))\right) \succeq\left(\sigma_{i}(P(m)), \nu_{i}(P(m))\right)
$$

$\forall i \in S$, con igualdad para todos los estados si y solo si $P(m+1)=P(m)$.

\section{Demostración:}

Supongamos que $P(m+1) \neq P(m)$. Sea $\left(\sigma_{i}(P(m)), \nu_{i}(P(m))\right):=(\rho, \eta)$ 
para $i \in I_{t}(m)$. Con el motivo de simplificar la notación, defínanse los siguientes tres conjuntos:

$$
A:=I_{t}(m), \quad D:=D(m), \quad y \quad E:=\left(\bigcup_{l=t}^{r_{m}} I_{l}(m) \backslash D(m)\right) .
$$

Obsérvese que cada estado en $D$ tiene acceso a algún estado en $A$ bajo $P(m+1)$. Como $P(m+1)_{i}=P(m)_{i}$ para $i \in A$, se sigue que cada clase final $C$ de $P(m+1)^{D}$, contiene una clase final(básica) $B$ de $P(m)^{A}$, por lo cual se tienen dos casos:

\section{Caso 1.}

$B \subsetneq C$, se tiene que:

$$
\sigma\left(P(m+1)^{C}\right)>\sigma\left(P(m+1)^{B}\right)=\sigma\left(P(m)^{B}\right)=\rho .
$$

Esto por la proposición (2.2.4) sobre los menores principales y el hecho de que $P(m+1)_{i}=P(m)_{i}$ para $i \in B$.

\section{Caso 2.}

$B=C$ se sigue que:

$$
\sigma\left(P(m+1)^{C}\right)=\sigma\left(P(m)^{B}\right)=\rho .
$$

En donde se tiene que $C$ solo tiene acceso a clases en $E$ (bajo $P(m+1)$ ). Si $\sigma\left(P(m+1)^{C}\right)=\rho$, se sigue entonces que $C$ es igual $B$, y para este caso $\nu_{i}(P(m+1))=\nu_{i}(P(m))$ para $i \in C$. De donde se obtiene que:

$$
\left(\sigma_{i}(P(m+1)), \nu_{i}(P(m+1))\right) \succeq(\rho, \eta)
$$

al menos para todos los estados $i$ que pertenecen a una clase final de $P(m+1)^{D}$, por la forma que tiene $D$; se sigue que lo anterior se cumple para todos los estados.

Como $P(m+1)_{i}=P(m)_{i}$ para $i \in E$, se tiene que (2.9) se cumple para 
todos los estados en $E$. Más aún, también se tiene que por (2.9) se puede extender para todo $i \in S$, ya que $P(m+1)_{i}=P(m)_{i}$ para $i \in S \backslash D$.

Por lo anterior; la igualdad en (2.9) se cumple para todos los estados si y solo si $A \backslash D=I_{t}(m) \backslash D(m)=\emptyset$, es decir, $P(m+1)=P(m)$. Una vez realizado lo anterior, es decir, la completación $\overline{P(0)}$ de $P(0)$, (una descomposición triangular en bloques), el siguiente paso es encontrar los eigenvectores.

\section{Procedimiento de perfección}

\section{Comenzar:}

Sea $\bar{P}(0)$ el resultado del procedimiento de completación y sea

$\left\{I_{1}(0), \ldots, I_{r_{0}}(0)\right\}$ que denota la partición espectral de $S$ con respecto a $\overline{P(0)}$ y sea $\left\{u(0)^{k} ; k=1,2, \ldots, r_{0}\right\}$ el conjunto de eigenvectores derechos asociados, dados por el Lema 2.2.19 (c).

Tómese $P\left(r_{0}+1\right)=\bar{P}(0)$. Para $m=r_{0}, r_{0}-1, \ldots, 2,1$, y aplíquese el siguiente paso de perfección:

\section{Paso de perfección}

Supóngase que se ha obtenido $P(m+1)$ entonces determínese $\tilde{P} \in \mathbb{K}$ de tal forma que:

$$
\sum_{j \in I_{m}(0)} \tilde{P}_{i j} u(0)_{j}^{(m)}=\operatorname{máx}_{P \in \mathbb{K}} \sum_{j \in I_{m}(0)} P_{i j} u(0)_{j}^{(m)},
$$

$i \in I_{m}(0)$, con $\tilde{P}_{i}:=\bar{P}(0)_{i}$, si

$$
\sum_{j \in I_{m}(0)} \tilde{P}(0)_{i j} u(0)_{j}^{(m)}=\operatorname{máx}_{P \in \mathbb{K}} \sum_{j \in I_{m}(0)} P_{i j} u(0)_{j}^{(m)} .
$$

Defínase $P(m)$ por: 


$$
P(m)_{i}:= \begin{cases}\tilde{P}(0)_{i} & i \in \bigcup_{k=1}^{m-1} I_{k}(0), \\ \tilde{P}_{i} & i \in I_{m}(0), \\ P(m+1)_{i} & i \in \bigcup_{k=m+1}^{r_{m}} I_{k}(0) .\end{cases}
$$

Pare: A $P(1)$ se le conoce como el perfeccionamiento de $\bar{P}(0)$.

Para demostrar que $P(1)$ es realmente un perfeccionamiento de $\bar{P}(0)$, se presentan los siguientes dos lemas.

Lema 2.4.4. Dado el procedimiento de perfección, se tiene que para $m=$ $r_{0}, r_{0}-1, \ldots, 2,1$ :

$$
\left(\sigma_{i}(P(m)), \nu_{i}(P(m))\right) \succeq\left(\sigma_{i}(P(m+1)), \nu_{i}(P(m+1))\right),
$$

$i \in S$.

\section{Demostración:}

Sea $\left(\sigma_{i}(P(0)), \nu_{i}(P(0))\right):=(\rho, \eta)$ para $i \in I_{m}(0)$. Para simplificar la notación se define:

$$
A:=I_{m}(0), \quad E:=\bigcup_{k=m+1}^{r_{0}} I_{k}(0)
$$

Sea $C$ una clase final de $P(m)^{A}$. Por $(2.10)$ se tiene que:

$$
\sum_{j \in A} p(m)_{i j} u(0)_{j}^{(m)} \geq \sum_{j \in A} \bar{p}(0)_{i j} u(0)_{j}^{(m)}=\rho u(0)_{i}^{m}
$$

con $i \in C$, dado lo anterior, por el lema 2.2.10 se sigue que: $\sigma\left(P(m)^{C}\right) \geq \rho$. Si se da el caso en que: $\sigma\left(P(m)^{C}\right)=\rho$ entonces por el lema 2.2.11 se obtiene que:

$$
\sum_{j \in A} p(m)_{i j} u(0)_{j}^{(m)}=\sum_{j \in C} p(m)_{i j} u(0)_{j}^{(m)}=\rho u(0)_{i}^{m}=\sum_{j \in A} \bar{p}(m)_{i j} u(0)_{j}^{(m)},
$$


para todo $i \in C$, lo que implica que:

$$
P(m)_{i}=\bar{P}(0)_{i}=P(m+1)_{i}
$$

con $i \in C$. Más aún, $C$ sólo tiene acceso posible a las clases de $E$ bajo $P(m)$. Como el mismo argumento funciona para cualquier clase final de $P(m)^{A}$ se sigue por (2.11), que lo anterior se cumple para todos los estados en $A$ y continuando con el proceso, se puede extender para cualquier estado $i \in S$.

El siguiente lema permite observar que sucede cuando se da la igualdad en el Lema 2.11

Lema 2.4.5. Sea $\mathbb{K}$ un conjunto finito de matrices cuadradas, no negativas con la propiedad producto. Sea $P(0)$ una matriz con radio espectral $\sigma$, con eigenvector asociado u(0), estrictamente positivo. Determinese P(1) de tal forma que lo siguiente se cumpla:

$$
P(1) u(0)=\operatorname{máx}_{P \in \mathbb{K}} P u(0)
$$

$\operatorname{con} P(1)_{i}:=P(0)_{i}$ si

$$
(P(0) u(0))_{i}=\left(\operatorname{máx}_{P \in \mathbb{K}} P u(0)\right)_{i} .
$$

Supóngase además que:

$$
\left(\sigma_{i}(P(1)),\left(\nu_{i}(P(1))\right)\right)=(\sigma, 1)
$$

$\forall i \in S$. Entonces debe existir un eigenvector $u(1)$ estrictamente positivo para $P(1)$, asociado a $\sigma$, tal que $u(1)_{i}=u(0)_{i}$ para $i$ en una clase básica de $P(1)$. Más aún $u(1) \geq u(0)$ con igualdad si y solo si $P(1)=P(0)$.

\section{Demostración:}

Como (2.12) se cumple, entonces las clases básicas de $P(1)$ son precisa- 
mente sus clases finales. De donde, para $P(1)$ existe un eigenvector estrictamente positivo, llamémosle $u(1)$, el cual está asociado a $\sigma$. Así como en la prueba del Lema 2.4.4, se tiene que para este caso $P(1)_{i}=P(0)_{i}$ para $i$ en una clases final de $P(1)$. De aquí, se tiene que una clase básica (final) de $P(1)$ es también una clase básica (final) de $P(0)$, por lo que $u(1)$ puede ser elegido de tal forma que $u(1)_{i}=u(0)_{i}$, para $i$ que pertenece a una clase básica de $P(1)$.

Sea $A$ que denota al conjunto de estados que no pertenecen a una clase básica de $P(1)$. Obsérvese lo siguiente:

Como $P(1)(u(0)-u(1)) \geq \rho(u(0)-u(1))$, se sigue que $u(1)_{i}=u(0)_{i}$ para $i \in S \backslash A$. Dado lo anterior se obtiene que:

$$
P(1)^{A}\left(u(0)^{A}-u(1)^{A}\right) \geq \rho\left(u(0)^{A}-u(1)^{A}\right)
$$

. Si $u(1)_{i}<u(0)_{i}$, para algún $i \in A$. Por el Lema 2.8 esto implicaría que $\left(P(1)^{A}\right) \geq \rho$, lo cual contradice la definición de $A$. Por tanto se tiene que $u(1) \geq u(0)$; sí se da el caso que $u(1)_{i}=u(0)_{i}$, para $i \in S \backslash A$ esto implica que $u(1)=u(0)$ lo cual dice que:

$$
\operatorname{máx}_{P \in \mathbb{K}} P u(0)=P(1) u(0)=\sigma u(0)=P(0) u(0),
$$

es decir $P(1)=P(0)$.

Ahora llevando lo anterior al Lema 2.4.4, se sigue que si la igualdad se cumple en (2.11) para algún $m \in r_{0}, r_{0}-1, \ldots, 2,1$ y además para todo $i \in S$, entonces existe un vector $u(1)^{(m)}$, definido en $I_{m}(0)$, el cual es estrictamente positivo y además cumple que:

$$
\sum_{j \in I_{m}(0)} p(m)_{i j} u(1)_{j}^{(m)}=u(1)_{i}^{(m)}
$$


$i \in I_{m}(0), \mathrm{y}$

$$
u(1)_{i}^{(m)} \geq u(0)_{i}^{(m)}
$$

$i \in I_{m}(0)$, con igualdad para todos los estados $i$ que pertenecen a cualquier clase $C \subset I_{m}(0)$ de $P(m)$ que satisfacen $\sigma\left(P(m)^{C}\right)=\rho$ ( $\rho$ definido como en la prueba del Lema 2.4.4).

Se tiene, para este caso que $P(m)=P(m+1)$ si y solo si $u(1)^{(m)}=u(0)^{(m)}$, y se obtiene:

$$
\left.\sum_{j \in I_{m}(0)} P^{(m}\right)_{i j} u(0)_{j}^{(m)}=\operatorname{máx}_{P \in \mathbb{K}} \sum_{j \in I_{m}(0)} P_{i j} u(0)_{j}^{(m)}=\rho u(0)_{i}^{(m)},
$$

$i \in I_{m}(0)$. Cabe notar que la prueba del Lema 2.3.1 consiste en un método iterativo en donde cada paso es un proceso de perfeccionamiento, aunque lo que es importante resaltar es que una vez terminado el procedimiento de perfeccionamiento se necesita el procedimiento de completación, debido a que no necesariamente se cumple que la partición espectral de $S$, coincide con la partición espectral de $S$ con respecto al proceso de perfeccionamiento. Los pasos anteriores se pueden resumir como sigue a continuación:

\section{Nota:}

\section{(a) Proceso iterativo de crecimiento óptimo}

\section{Comenzar:}

Elijase $P(0) \in \mathbb{K}$.

Para $m=0,1,2, \ldots$, encuentre la matriz $P(m+1)$ con la siguiente regla:

Paso iterativo Calcule $\bar{P}(m)$, la completación de $P(m)$.

Encuentre $P(m+1)$, el perfeccionamiento de $P(m)$.

Pare Si $P(m+1)=P(m)$. Entonces se define $\left\{I_{1}(m), \ldots, I_{r_{m}}(m)\right\}$ como la partición espectral de $S$ con respecto a $P(m)$ y sea $\left\{u^{(1)}, \ldots, u^{\left(r_{m}\right)}\right\}$ el conjunto de eigenvectores estrictamente positivos descritos en el 
Lema 2.2.19. Entonces defínase $\hat{P}:=P(m), r:=r_{m},\{I(1), \ldots I(r)\}:=$ $\left\{I_{1}(m), \ldots, I_{r_{m}}(m)\right\}$ y $\left\{u^{(1)}, \ldots, u^{(r)}\right\}:=\left\{u^{(1)}, \ldots, u^{\left(r_{m}\right)}\right\}$.

(b) Obsérvese que los lemas precedentes demuestran que el procedimiento anterior no se cicla y que además termina en un número finito de pasos, esto debido a que $\mathbb{K}$ es finito.

Así que para recapitular todo lo hecho hasta este instante, se enuncia el siguiente teorema, el cual, su demostración se sigue directamente de aplicar los métodos de completación y perfeccionamiento.

Teorema 2.4.6. Sea $\mathbb{K}$ un conjunto finito de matrices cuadradas, no negativas y con la propiedad producto. Sean $\sigma:=\operatorname{máx}\{\sigma(P) \mid P \in \mathbb{K}\}$ y $\nu:=\operatorname{máx}\{\nu(P) \mid P \in \mathbb{K}, \sigma(P)=\hat{\sigma}\}$. Entonces existe una partición $\{D(\nu), D(\nu-1), \ldots, D(1), D(0)\}$ del espacio de estados $S$ tal que lo siguiente se cumple:

(a) Sea $P^{(k, l)}$ que denota la restricción de $P$ a $D(k) \times D(l)$ entonces $P^{(k, l)}=0$ para $k<l, k, l=0,1, \ldots, \nu . y P \in \mathbb{K}$.

(b) Existe una matriz $\hat{P} \in \mathbb{K}$ con $\sigma(\hat{P})=\hat{\sigma}$ y $\nu(\hat{P})=\nu$ y vectores estrictamente positivos $\hat{u}^{(k)}$ definidos en $D(k)$ de tal forma que:

$$
\hat{P}^{(k, k)} \hat{u}^{(k)}=\operatorname{máx}_{P \in \mathbb{K}} P^{(k, k)} \hat{u}^{(k)}=\hat{\sigma} \hat{u}^{(k)}
$$

$k=1,2, \ldots, \nu$.

Para cada $k=0,1, \ldots, \nu$, el conjunto $D(k)$ representa la unión de todas clases con profundidad $k$ con respecto a $\hat{P}$. Más aún,

$$
\operatorname{máx}_{P \in \mathbb{K}} \sigma\left(P^{(0,0)}\right)<\hat{\sigma}
$$


(c) Elijase $x(0)>0$ y sea $x(n)$ definido por:

$$
x(n)=\operatorname{máx}_{P \in \mathbb{K}} P x(n-1),
$$

$n \in \mathbb{N}$. Entonces existen constantes $c_{1}, c_{2}>0$ tal que:

$$
c_{1} \hat{u}_{i}^{(k)} \leq\left(\begin{array}{c}
n \\
k-1
\end{array}\right)^{-1} \hat{\sigma}^{-n} x(n)_{i} \leq c_{2} \hat{u}_{i}^{(k)}
$$

para $i \in D(k) ; k=1, \ldots, \nu$, asi mismo el

$$
\lim _{n \rightarrow \infty} \hat{\sigma}^{-n} x(n)_{i}=0
$$

para $i \in D(0)$.

Definición 2.4.7. La partición $\{D(\nu), D(\nu-1), \ldots, D(1), D(0)\}$ la cual se introdujo en el Teorema 3.6 se le conoce como la partición principal de $S$ con respecto a $\mathbb{K}$. 


\section{Capítulo 3}

\section{Convergencia de Métodos Recursivos}

Este capítulo se centra en el hecho de analizar que pasa cuando se toma el límite de la recursión (3.1), dada a continuación, para los casos en el que el conjunto restricción consta de matrices reducibles ó irreducibles y además se tiene por restricción que el grado $\nu$ de todas las matrices es igual a 1.

\subsubsection{Convergencia del Caso $\nu=1$ usando Progra- mación Dinámica}

Sea

$$
x(n)=\operatorname{máx}_{P \in \mathbb{K}} P x(n-1),
$$

$n \in \mathbb{N}, x(0)>0$. Tómese en particular, la partición principal de $S$ con respecto a $\mathbb{K}$ y sea $\hat{\sigma}$ que denota: $\hat{\sigma}:=\operatorname{máx}_{P \in \mathbb{K}} \sigma(P)$. Lo que se afirma es que existen cotas superiores e inferiores para la sucesión:

$$
\left(\begin{array}{c}
n \\
k-1
\end{array}\right)^{-1} \hat{\sigma}^{-n} x(n)_{i}
$$


$i \in D(k) k=1, \ldots, \nu$.

Aunque lo anterior nos da una idea sobre el comportamiento asintótico, un punto a destacar es que si la matriz tiene ciertas propiedades entonces se puede decir cual es el límite de la recursión.

Por ejemplo, si se considera una matriz cuadrada, irreducible y aperiódica entonces se tiene que:

$$
\lim _{n \rightarrow \infty} \sigma^{-n} P^{n} x(0)
$$

existe y es estrictamente positivo si $x(0) \geq 0$; lo cual se basa en el hecho de la forma canónica de Jordan de la matriz P, pero para la técnica de las matrices no negativas se sigue directamente del Lema 2.2.9.

En lo que concierne a esta descripción lo importante es utilizar el trabajo realizado hasta este instante y adaptarlo para el caso recursivo al cual nos estamos enfrentando.

Para este problema, se está considerando que la partición principal de $S$ con respecto a $\mathbb{K}$ viene dada de la forma $\{D(1), D(0)\}$, esto debido a que se está suponiendo que $\nu=1$, lo que de otra forma se puede expresar como:

$$
\operatorname{máx}\{\nu(P) \mid P \in \mathbb{K}, \sigma(P)=\hat{\sigma}\}=1 \text {, }
$$

en donde:

$$
\hat{\sigma}:=\operatorname{máx}\{\sigma(P) \mid P \in \mathbb{K}\} \text {. }
$$

\subsubsection{Recursión de Programación Dinámica con Ma- trices no Negativas e Irreducibles}

En este caso, cabe señalar que lo primero que hay que demostrar es: ¿en qué tipo de matrices se cumple la existencia del límite de la recursión (3.1)? .

Para este objetivo se comenzará con el siguiente lema. 
Lema 3.0.8. Sea P una matriz no negativa, cuadrada, aperiódica e irreducible con radio espectral $\sigma$ y sea $\{x(n) ; n=0,1,2, \ldots$,$\} un conjunto$ de vectores tales que $\sigma^{-n} x(n)$ está acotado (uniformemente para $n \in \mathbb{N}$ ). Además supóngase que:

$$
x(n+1) \geq P x(n),
$$

$n \in \mathbb{N}$. Entonces existe un vector $x$ el cual cumple:

$$
\lim _{n \rightarrow \infty} \sigma^{-n} x(n)=x=\sigma^{-1} P x .
$$

Más aún $x(0) \geq 0$ implica que $x>0$.

\section{Demostración:}

Como $\{x(n) ; n=0,1,2, \ldots$,$\} está acotado entonces se puede suponer la$ existencia de un número finito de puntos límites para esta sucesión. Así que supóngase que existen dos puntos límites diferentes $a$ y $b$. Si se hace un proceso iterativo para (3.4) se tiene que:

$$
\sigma^{-(n+m)} x(n+m) \geq \sigma^{-m} P^{m} \sigma^{-n} x(n),
$$

$n, m \in \mathbb{N}$. Tómese un $n$ fijo y sea $m_{1}, m_{2}, \ldots$ una sucesión de tal forma que:

$$
\lim _{k \rightarrow \infty} \sigma^{n+m_{k}} x\left(n+m_{k}\right)=a .
$$

Por el Lema 2.2.9 se obtiene que lo anterior implica que $a \geq P^{*} \sigma^{-n} x(n)$. Si se hace lo mismo pero ahora con $b$ para una sucesión $n_{k}$, se tiene que:

$$
\lim _{k \rightarrow \infty} \sigma^{n+n_{k}} x\left(n+n_{k}\right)=b .
$$

Por otro lado se obtiene que:

$a \geq P^{*} b$ y análogamente se obtiene que $b \geq P^{*} a$. Si se juntan las dos 
desigualdades anteriores resulta que:

$$
a \geq P^{*} P^{*} a=P^{*} a
$$

y como

$$
P^{*} \geq 0, a \geq P^{*} a
$$

implica que

$$
0<P^{*}\left(a-P^{*} a\right)=P^{*} a-P^{*} a=0
$$

lo cual es una contradicción. Si se realiza el mismo argumento para $b$ se tiene que $a \geq b$ y que $b \geq a$ por lo tanto $a=b$ y así el límite es único. Como $a \geq P^{*} x(0)$ entonces se tiene que $a>0$ si $x(0) \geq 0$.

Teorema 3.0.9. Sea $\mathbb{K}$ un conjunto finito de matrices cuadradas no negativas e irreducibles, con la propiedad producto. Sea $\hat{\sigma}:=\operatorname{máx}\{\sigma(P) \mid P \in$ $\mathbb{K}\}$ y supóngase que existe al menos una matriz $\bar{P} \in \mathbb{K}$ con $\sigma(\bar{P})=\hat{\sigma}$ $y$ que ésta es aperiódica. Elijase $x(0) \geq 0$ y sea $x(n)$ como en (3.1) para $n=1,2, \ldots$ Entonces:

$$
\lim _{n \rightarrow \infty} \sigma^{-n} x(n)
$$

Sea $x$ que denota este límite. Entonces $x \geq 0$ y se tiene además que:

$$
\operatorname{máx}_{P \in \mathbb{K}} P x=\hat{\sigma} x
$$

\section{Demostración:}

Por el Lema 2.3.1 existe un vector $\hat{u} \geq 0$ tal que:

$$
\operatorname{máx}_{P \in \mathbb{K}} P \hat{u}=\hat{\sigma} \hat{u}
$$

sin pérdida de generalidad, se puede suponer que

$$
\hat{u} \geq x(0)
$$


de lo anterior se sigue que:

$$
0 \leq \hat{\sigma}^{-n} x(n) \leq \hat{u}
$$

$n \in \mathbb{N}$. Como $x(n+1) \geq \bar{P} x(n)$ para todo $n$, por el Lema 3.0.8 se tiene que el límite dado por: $x:=\lim _{n \rightarrow \infty} \sigma^{-n} x(n)$ existe. De lo anterior se sigue que la fórmula (3.6) se transforma a:

$$
\hat{\sigma} \cdot \hat{\sigma}^{-(n+1)} x(n+1)=\operatorname{máx}_{P \in \mathbb{K}} P \hat{\sigma}^{-n} x(n),
$$

$n \in \mathbb{N}$. Si se hace tender $n \rightarrow \infty$ en los dos lados de la igualdad, se obtiene el resultado, más aún, por el Lema 3.0.8 se tiene que $x \geq 0$.

Teorema 3.0.10. Sea $\mathbb{K}$ un conjunto finito de matrices cuadradas, no negativas e irreducibles, con la propiedad producto. Sea $\hat{\sigma}:=\operatorname{máx}\{\sigma(P) \mid P \in$ $\mathbb{K}\}$, sea $d(P)$ que denota el período de $P$, (recuérdese que d se define por: $d:=$ m.c.d. $\{d(P) \mid P \in \mathbb{K}, \sigma(P)=\hat{\sigma}\})$. Elíjase $x(0) \geq 0$ y sea $x(n)$ definido como en (3.1), para $n=1,2, \ldots$ Entonces para $l=0,1, \ldots d-1$.

$$
\lim _{n \rightarrow \infty} \sigma^{-l+k d} x(l+k d),
$$

existe y es estrictamente positivo.

\section{Demostración:}

Se sigue del teorema anterior sólo ajustando los pasos para el período de P. $\square$.

\subsubsection{Recursión con Programación Dinámica de Ma- trices no Negativas y Reducibles}

En este apartado el objetivo será demostrar que la recursión (3.1) para un conjunto de matrices cuadradas, no negativas, reducibles y con la propiedad producto están acotadas, en algún sentido, siempre y cuando la condición (3.2) se cumpla. 
Lema 3.0.11. Sea $\mathbb{K}$ un conjunto finito de matrices cuadradas, no negativas y que cumplen con la propiedad producto, sea $\hat{\sigma}:=\operatorname{máx}\{\sigma(P) \mid P \in \mathbb{K}\}$. Entonces:

(a) $\hat{\sigma}=\inf \left\{\lambda \mid \exists w \geq 0\right.$ tal que $\left.\operatorname{máx}_{P \in \mathbb{K}} P w \leq \lambda w\right\}$.

(b) Para cada $\lambda>\hat{\sigma}$ existe un vector $w(\lambda)>0$ tal que:

$$
\max _{P \in \mathbb{K}} P w(\lambda)<\lambda w(\lambda)
$$

\section{Demostración:}

La primera parte del lema se sigue inmediatamente del Lema 2.2.10; la segunda parte, se sigue de tomar $w(\lambda):=\operatorname{máx}_{P \in \mathbb{K}}(\lambda I-P)^{-1} e$ (en donde $e$ denota al vector con todas sus entradas igual a 1 ).

El siguiente lema establece una cota para la sucesión $\hat{\sigma}^{-n} x(n)$.

Lema 3.0.12. Sea $\mathbb{K}$ un conjunto finito de matrices cuadradas, no negativas y que cumplen con la propiedad producto. Sea $0<\hat{\sigma}:=\operatorname{máx}\{\sigma(P) \mid P \in$ $\mathbb{K}\}$ y sea $S$ la partición principal con respecto a $\mathbb{K}$ dada por $(\{D(1), D(0)\})$, lo anterior es debido a que se está asumiendo (3.2). Entonces para $x(n)$ definido como en (3.1) se tiene que:

$$
\lim _{n \rightarrow \infty} \hat{\sigma}^{-n} x(n)_{i}=0
$$

$i \in D(0)$.

$$
c_{1} \hat{u}_{i}^{(1)} \leq \hat{\sigma}^{-n} x(n)_{i} \leq c_{2} \hat{u}_{i}^{(1)},
$$

$n \in \mathbb{N} ; i \in D(1)$, para algunas constantes $c_{1}, c_{2}$ y $\hat{u}$ definido como en el Teorema 2.4.6.

\section{Demostración:}

Sea $P \in \mathbb{K}$ y sea $P^{(k, l)}$ la restricción de $P$ a $D(k) \times D(l)$, para $k, l=0,1$. 
Se tiene que $P^{(0,1)}=0$ para toda $P$. Como el máx $\left\{\sigma\left(P^{(0,0))} \mid P \in \mathbb{K}\right\}<\hat{\sigma}\right.$ entonces existe $\lambda \leq \hat{\sigma}$ y un vector $w(\lambda)>0$ definido sobre $D(0)$ de tal forma que:

$$
\operatorname{máx}_{P \in \mathbb{K}} P^{(0,0)} w(\lambda) \leq \lambda w(\lambda) .
$$

Eligiendo $c>0$ tal que

$$
x(0)_{i} \leq c w(\lambda)_{i}
$$

para $i \in D(0)$, se obtiene que:

$$
\hat{\sigma}^{-n} x(n)_{i} \leq c \lambda^{n} \sigma^{-n} w(\lambda)
$$

$n \in \mathbb{N} ; i \in D(0)$, con lo cual se demuestra (3.7) ya que $x(n)>0$ para todo $n$. Tómese $c_{1}>0$ de tal forma que:

$$
c_{1} \hat{u}_{i}^{(1)} \leq x(0)_{1}
$$

$i \in D(1)$ y recordando que se cumple:

$$
\operatorname{máx}_{P \in K} P^{(1,1)} \hat{u}^{(1)}=\hat{\sigma} \hat{u}^{(1)}
$$

nótese que la desigualdad de lado izquierdo de (3.8) se sigue directamente del hecho que:

$$
x(n)^{(1)} \geq \operatorname{máx}_{P \in K} P^{(1,1)} x(n-1)^{(1)},
$$

$n=1,2, \ldots$, en donde $x(n)^{(1)}$ representa la restricción de $x(n)$ a $D(1)$. Por último si se toma $w(\lambda)>0$ y $\alpha \in R$ tal que:

$$
\begin{gathered}
\operatorname{máx}_{P \in K} P^{(1,0)} w(\lambda) \leq \hat{u}^{(1)}, \\
x(0)_{i} \leq \alpha w(\lambda)_{i},
\end{gathered}
$$

$i \in D(0)$,

$$
x(0)_{i} \leq \alpha \hat{u}_{i}^{(1)}
$$


$i \in D(1)$, usando inducción se tiene que:

$$
x(n)_{i} \leq \alpha\left\{\hat{\sigma}^{n}+\hat{\sigma}^{n-1} /(1-\lambda \hat{\sigma})^{-1}\right\} \hat{u}_{i}^{(1)} \leq c_{2} \sigma^{n} \hat{u}_{i}^{(1)},
$$

$i \in D(1)$, para un valor apropiado de $c_{2}$.

Definición 3.0.13. Una matriz no negativa $P$ se dice que es aperiódica si la restricción a cada una de sus clases básicas es aperiódica.

Nota: Cabe mencionar que la definición que se está dando ahora sobre aperiodicidad depende sobre las clases básicas y es una aplicación de la definición dada en el capítulo de cadenas de Markov.

Teorema 3.0.14. Sea $\mathbb{K}$ un conjunto finito de matrices cuadradas, no negativas y que cumplen con la propiedad producto. Sea $\hat{\sigma}:=\operatorname{máx}\{\sigma(P) \mid P \in$ $\mathbb{K}\}$ y sea S la partición principal con respecto a $\mathbb{K}$ dada por $(\{D(1), D(0)\})$. También supóngase que para cada matriz $P \in \mathbb{K}$ para la cual se cumple que $\sigma(P)=\hat{\sigma}$, es aperiódica. Entonces existe un vector $x \geq 0$ con $x_{i}=0$ para $i \in D(0)$ y $x_{i}>0$ para $i \in D(1)$ tal que

$$
\lim _{n \rightarrow \infty} \hat{\sigma}^{-n} x(n)=x
$$

en donde $x(n)$ se define como en (3.1); más aún el vector $x$ cumple:

$$
\operatorname{máx}_{P \in \mathbb{K}} P x=\hat{\sigma} x
$$

\section{Demostración:}

Defínase $\bar{x}(n):=\hat{\sigma}^{-n} x(n)$; por el Lema 3.0.12 se tiene que:

$$
\lim _{n \rightarrow \infty} \bar{x}(n)_{i}=0,
$$

$i \in D(0)$. Por lo que los estados importantes son los que se encuentran en $D(1)$. Como (3.8) se cumple entonces se pueden definir vectores $a$ y $b$, componente a componente tales que: 


$$
\begin{aligned}
a & :=\limsup _{n \rightarrow \infty} \bar{x}(n), \mathrm{y} \\
b & :=\liminf _{n \rightarrow \infty} \bar{x}(n) .
\end{aligned}
$$

Supóngase que $a \geq b$ (nótese que $b \geq 0$ y que $a_{i}=b_{i}=0$ para $i \in D(0)$ ). Tómese una subsucesión $\left\{n_{k} ; k=0,1,2, \ldots,\right\}$ tal que:

$\lim _{k \rightarrow \infty} \bar{x}\left(n_{k}\right)=b$ y de tal forma que $\lim _{k \rightarrow \infty} \bar{x}\left(n_{k}-1\right)$ existe. Defínase $\bar{x}:=$ $\lim _{k \rightarrow \infty} \bar{x}\left(n_{k}-1\right)$. Obsérvese que $\bar{x} \geq b$ y que $\bar{x}_{i}=b_{i}=0$ para $i \in D(0)$. Como $\mathbb{K}$ es finito, existe una matriz $\bar{P}$ y una subsucesión $\left\{n_{k_{(l)}} ; l=0,1,2, \ldots\right\}$ de $\left\{n_{k} ; k=0,1,2, \ldots,,\right\}$ para $l=0,1,2, \ldots$.

$$
\bar{x}\left(n_{k(l)}\right)=\hat{\sigma}^{-1} \bar{P} \bar{x}\left(n_{k(l)}-1\right)=\hat{\sigma}^{-1} \operatorname{máx}_{P \in \mathbb{K}} P \bar{x}\left(n_{k(l)}-1\right) .
$$

Si se hace tender $l \rightarrow \infty$ en (3.11) se tiene:

$$
b=\hat{\sigma}^{-1} \bar{P} \bar{x} \geq \hat{\sigma}^{-1} \bar{P} b
$$

Análogamente, se tiene que para una $\tilde{P} \in \mathbb{K}$,

$$
a \leq \hat{\sigma}^{-1} \tilde{P} a
$$

Como $\tilde{P}$ se encontró en forma óptima, (3.11) implica directamente que:

$$
b \geq \hat{\sigma}^{-1} \tilde{P} \bar{x} \geq \hat{\sigma}^{-1} \tilde{P} b
$$

Combinando las ecuaciones anteriores se concluye que:

$$
a-b \leq \hat{\sigma}^{-1} \tilde{P}(a-b)
$$

y como para $i \in D(0), a_{i}=b_{i}=0$ entonces (3.12) se reduce a $i \in D(1)$, es decir:

$$
a^{(1)}-b^{(1)} \leq \hat{\sigma}^{-1} \tilde{P}^{(1,1)}\left(a^{(1)}-b^{(1)}\right),
$$

en donde $a^{(1)}$ y $b^{(1)}$ denota la restricción de $a$ y $b$ a $D(1)$. Como se supuso que $b \leq a$, por el Lema 2.2.12 se tiene que $\sigma\left(\tilde{P}^{(1,1)}\right)=\hat{\sigma}$. Más aún se tiene 
que existe un vector $\hat{u}^{(1)}>0$, definido en $D(1)$ tal que:

$$
\tilde{P}^{(1,1)} \hat{u}^{(1)} \leq \operatorname{máx}_{P \in \mathbb{K}} P^{(1,1)} \hat{u}^{(1)}=\hat{\sigma} \hat{u}^{(1)} .
$$

Lo anterior se tiene por el resultado 2.4.6 (b). Esto implica que cada clase básica de $\tilde{P}^{(1,1)}$ es final. Sea $C$ cualquier clase básica de $\tilde{P}^{(1,1)}$, como

$$
\left(\tilde{P}^{(1,1)}\right)^{C} x(n-1)^{C} \leq x(n)^{(C)}
$$

para toda $n \in \mathbb{N}$, y además $\sigma\left(\tilde{P}^{C}\right)=\hat{\sigma}$, por el Lema 3.0.8, se sigue que $a_{i}=b_{i}$ para $i \in C$.

Por último, sea $E$ que denota el conjunto de los estados en $D(1)$ que no están contenidos en alguna clase básica de $\tilde{P}^{(1,1)}$; por lo anterior se tiene que si $b \leq a$ implica que $b^{E} \leq a^{E}$ y por (3.12) se obtiene:

$$
a^{E}-b^{E} \leq \hat{\sigma}^{-1} \tilde{P}^{E}\left(a^{E}-b^{E}\right),
$$

lo cual tiene como resultado que $\sigma\left(\tilde{P}^{E}\right) \geq \sigma$ por el Lema 2.2.12. De donde, se contradice la definición de $E$, por lo tanto $a=b$. Por (3.8) se tiene que $a_{i}>0$ para $i \in D(1)$. Finalmente por (3.10) se sigue que:

$$
\hat{\sigma} \bar{x}(n+1)=\operatorname{máx}_{P \in \mathbb{K}} P \bar{x}(n),
$$

haciendo tender $n$ a infinito.

Teorema 3.0.15. Sea $\mathbb{K}$ un conjunto finito de matrices cuadradas, no negativas y que cumplen con la propiedad producto, sea $\hat{\sigma}:=\operatorname{máx}\{\sigma(P) \mid P \in$ $\mathbb{K}\}$ y sea $S$ la partición principal con respecto a $\mathbb{K}$ dada por $(\{D(1), D(0)\})$. Supóngase también que cada matriz $P \in \mathbb{K}$ para la cual se cumple que $\sigma(P)=\hat{\sigma}$ es aperiódica. Entonces existen constantes $\rho<1$ y $c>0$ tal que:

$$
\|\hat{\sigma} x(n)-x\| \leq c \rho^{n}
$$


$n \in \mathbb{N}$, en donde $x:=\lim _{n \rightarrow \infty} \hat{\sigma}^{-n} x(n)$.

\section{Demostración:}

Sea $\bar{x}(n):=\hat{\sigma}^{-n} x(n)$ y sean $\bar{x}(n)^{k}, x^{(k)}$ que denotan la restricción de $\bar{x}(n), x$ a $D(k), k=\{0,1\}$. Como para algún $\lambda<\hat{\sigma}$ y algún $w(\lambda)>0$, definidos sobre $D(0)$, se tiene por el Lema 3.0.11 que:

$$
\operatorname{máx}_{P \in K} P^{(0,0)} w(\lambda) \leq \lambda w(\lambda)
$$

esto implica que para $i \in D(0)$ cuando $n \rightarrow \infty, \bar{x}(n)^{(0)}$ tiende a cero geométricamente (eligiendo $c>0$ de tal forma que: $\bar{x}(0)_{i} \leq c w(\lambda)_{i}$ para $i \in D(0)$ de donde: $\bar{x}(n)_{i} \leq c \lambda^{n} \hat{\sigma}^{-n} w(\lambda)_{i}$ para $\left.i \in D(0)\right)$. Más aún como $\lim _{n \rightarrow \infty} \bar{x}(n)=x$ y se cumple (3.10), entonces para $n \geq n_{0}$ se tiene que: $x(n+1)=\operatorname{máx}_{P \in K_{1}} P x(n)$, en donde $K_{1}$ se define por: $K_{1}:=\{P \in \mathbb{K} \mid P x=$ $\hat{\sigma} x\}$.

Por otro lado, para los estados $i \in D(1)$ y para $n \geq n_{0}$, la recursión (3.1) puede ser reescrita como:

$$
\bar{x}(n+1)_{i}=\operatorname{máx}_{P \in K_{1}}\left\{\sum_{j \in D(1)} \hat{\sigma}^{-1} P_{i j} \bar{x}(n)_{j}+\sum_{j \in D(0)} \hat{\sigma}^{-1} P_{i j} \bar{x}(n)_{j}\right\} .
$$

Por (3.10) y el hecho de que $x^{(1)}>0$, se tiene que la siguiente transformación puede ser aplicada:

$$
\tilde{P}_{i j}:=x_{i}^{-1} \hat{\sigma}^{-1} P_{i j} x_{j}
$$

$i, j \in D(1), P \in \mathbb{K}$

$$
\tilde{x}(n)_{i}:=x_{i}^{-1} \bar{x}(n)_{i},
$$

$i \in D(1), n \geq n_{0}$ y sea

$$
\tilde{r}(P, n)_{i}:=\hat{\sigma}^{-1} x_{i}^{-1} \sum_{j \in D(0)} P_{i j} \bar{x}(n)_{j},
$$


$i \in D(1), n \geq n_{0}, P \in \mathbb{K}$.

Lo cual permite transformar (3.13) como:

$$
\tilde{x}(n+1)_{i}=\operatorname{máx}_{P \in K_{1}}\left\{\sum_{j \in D(1)} \tilde{P}_{i j} \tilde{x}(n)_{j}+\tilde{r}(P, n)_{i}\right\},
$$

$i \in D(1), n \geq n_{0}$.

Como $\tilde{r}(P, n)_{i}$ tiende a cero geométricamente cuando $n \rightarrow \infty$ para cada $P \in K_{1}$ y para $i \in D(1)$. Como $\left\{\tilde{P}^{(1,1)} \mid P \in K_{1}\right\}$ es un conjunto de matrices estócasticas entonces por pág.77, [30], $\tilde{x}(n)_{i}$ converge a 1 geométricamente cuando $n \rightarrow \infty$, con $i \in D(1)$. De donde se obtiene $\bar{x}(n)^{(1)}$ tiende a $x^{(1)}$ geométricamente.

En el caso cuando $P$ es periódica existe una versión del teorema anterior, aunque para este trabajo sólo se enunciará. Sea $\hat{\sigma}:=\operatorname{máx}\{\sigma(P) \mid P \in$ $\mathbb{K}\}$, se define $\tilde{K}:=\{P \in \mathbb{K} \mid \sigma(P)=\hat{\sigma}\}$.

Tómese alguna $P \in \mathbb{K}$ y supóngase que se tienen $k$ clases básicas, llamémosle $B(1), \ldots, B(k)$, y sea $d_{l}(P)$ que denota el período de $P^{B_{(l)}}$ para $l=1, \ldots, k$. Defínase:

$$
d(P)=m . c \cdot m\left\{d_{1}(P), \ldots, d_{k}(P)\right\}
$$

lo cual puede ser hecho para cada $P \in \tilde{K}$ ya que $\tilde{K}$ es finito, y por último denótese

$$
d:=\operatorname{m.c.d}\{d(P) \mid P \in \tilde{K}\},
$$

dado lo anterior se esta en condiciones para formular el siguiente teorema.

Teorema 3.0.16. Sea $\tilde{K}, \hat{\sigma}$ y d como en la parte anterior, supóngase que la condición (3.2) se cumple. Entonces existen vectores $w(l) \geq 0(l=$ 
$0,1, \ldots, d-1)$ de tal forma que para $x(n)$ definido como en (3.1) lo siguiente se cumple:

$$
\lim _{n \rightarrow \infty} \hat{\sigma}^{-(l+k d)} x(l+k d)=w(l),
$$

$l=0,1, \ldots, d-1$. Más aún la siguiente ecuación se cumple:

$$
\operatorname{máx}_{P \in K_{1}} P w(l)=\hat{\sigma} w(l+1),
$$

$l=0,1 \ldots, d-1$. en donde $w(d):=w(0)$.

Nota:Existe una versión del teorema 3.0.15 para el caso general. Para este trabajo solo se enunciará aunque la demostración se puede encontrar en las pág.79-86 [31].

Teorema 3.0.17. Sea $\hat{\sigma}:=\operatorname{máx}\{\sigma(P) \mid P \in \mathbb{K}\}$ y sea $\{D(\nu), \ldots, D(1), D(0)\}$ la partición principal de $S$ con respecto a $\mathbb{K}, \nu \in \mathbb{N}$, en donde $\mathbb{K}$ es un conjunto de matrices no negativas con la propiedad producto. Supóngase además que para cada matriz $P \in \mathbb{K}$ tal que $\sigma(P)=\hat{\sigma}$ y $\nu(P)=\nu$ son aperiódicas. Entonces existen vectores $y(1), y(2), \ldots, y(\nu), y$ constantes $c>0, \rho<\hat{\sigma}$ tal que la sucesión

$$
x(n+1)=\operatorname{máx}_{P \in \mathbb{K}} P x(n)
$$

$x(0)>0 ; n \in \mathbb{N}$, cumple con:

$$
\left\|x(n)-\left\{\left(\begin{array}{c}
n \\
\nu-1
\end{array}\right) \hat{\sigma}^{n-\nu+1} y(\nu)+\ldots+\left(\begin{array}{c}
n \\
1
\end{array}\right) \hat{\sigma}^{n-1} y(2)+\hat{\sigma}^{n} y(1)\right\}\right\| \leq c \rho^{n}
$$

$n \in \mathbb{N}$. Además los vectores $y(k)$ satisfacen que:

$$
y(k)_{i}>0
$$

$i \in D(k), k=1, \ldots, v$

$$
y(k)_{i}=0,
$$


$i \in \bigcup_{l=0}^{k-1} D(l), k=1, \ldots, \nu$

Más aún, las siguientes relaciones se cumplen:

$$
\operatorname{máx}_{P \in \mathbb{K}} P y(\nu)=\hat{\sigma} y(\nu)
$$

$$
\operatorname{máx}_{P \in \mathbb{K}_{l+1}} P y(l)=\hat{\sigma} y(l)+y(l+1)
$$

$l=\nu-1, \nu-2, \ldots, 1$, con

$\left\{\begin{array}{l}K_{\nu}:=\{P \in \mathbb{K} \mid P y(\nu)=\hat{\sigma} y(n u)\} \\ K_{l}:=\left\{P \in \mathbb{K}_{l+1} \mid P y(l)=\hat{\sigma} y(l)+y(l+1)\right\} \quad l=\nu-1, \nu-2, \ldots, 2,1 .\end{array}\right.$ 


\section{Capítulo 4}

\section{Fundamentos de las}

\section{Aplicaciones a Control}

\section{Estocástico}

En este capítulo quedarán establecidos los preliminares que permiten plantear un problema de control óptimo. En la primera sección se establecerá lo que es un modelo de control de Markov (MCM). En la segunda sección se define lo que entenderemos por política y así llegar a la definición de proceso de control de Markov. En la tercera sección, se plantea de manera general lo que es un problema de control de Markov (PCM), o problema de control óptimo (PCO).

\subsection{Modelos de Control de Markov}

Definición 4.1.1. Un Modelo de Control de Markov es una quintúpla

$$
M:=(S, A, A(s) \mid s \in S, Q, \rho)
$$

que consiste de: 
1. $S$, un conjunto finito, al que llamaremos el espacio de estados del sistema. Los elementos de $s \in S$ se les conocerá como los estados.

2. A, un conjunto finito, llamado espacio de controles o espacio de acciones.

3. $\{A(s) \mid s \in S\}$, una familia de subconjuntos no vacíos de $A$, donde $A(s)$ es el conjunto de controles admisibles para el estado $s \in S$.

$$
\Theta:=\{(s, a) \mid s \in S, a \in A(s)\}
$$

A este conjunto se le llamará el conjunto de pares estado-acción admisibles.

4. $Q$, una medida de probabilidad sobre $S$ dado $\Theta$, que también es conocida como ley de transición. Esta forma de llamar a $Q$ tiene sentido debido a que nos da la probabilidad condicional de que el sistema se mueva a un nuevo estado, dado que se encuentra en el estado actual y se elige una acción admisible; es decir $Q$ viene dado por lo siguiente:

$$
Q(B \mid s, a):=\operatorname{Prob}\left(X_{n+1} \in B \mid X_{n}=s, a_{n}=s\right),
$$

$B \subset S$, donde para cada acción $n=0,1,2, \ldots, X_{n}$ y $a_{n}$ denotan el estado y la acción correspondiente, respectivamente.

5. $\rho: \Theta \rightarrow \mathbb{R}$, una función la cual representa la respuesta del sistema, en el sentido de que $\rho(s, a)$ resulta de haber aplicado el control $a$ cuando el sistema estaba en el estado $s$. 


\subsection{Políticas}

En este momento se introducirá un nuevo concepto para los modelos de control, lo que ayudará a definir un proceso de control de Markov. Considérese un modelo de control como en la definición (4.1). Para cada $n=0,1, \ldots$ se define el espacio $H_{n}$ de historias admisibles hasta el tiempo n como sigue:

$$
H_{0}:=S ; \text { y } H_{n}:=\Theta^{n} \times H_{n-1}
$$

para $n=1,2, \ldots$, en donde $\Theta$ es el definido en (4.2). Un elemento $h_{n}$ de $H_{n}$, al que se le conocerá como n-historia admisible o simplemente n-historia, es un vector de la forma:

$$
h_{n}=\left(x_{0}, a_{0}, \ldots, x_{n-1}, a_{n-1}, x_{n}\right)
$$

con $\left(x_{i}, a_{i}\right) \in \Theta$ para $i=0,1, \ldots, n-1$, y $x_{n} \in S$.

Definición 4.2.1. Una política es una sucesión $\pi=\left\{\pi_{n}, n=0,1, \ldots\right\}$ de medidas de probabilidad $\pi_{n}$ sobre el conjunto de controles $A$ dado $H_{n}$ que satisfacen lo siguiente:

$$
\pi_{n}\left(A\left(x_{n}\right) \mid h_{n}\right)=1
$$

para todo $h_{n} \in H_{n}, n=0,1, \ldots$

Definición 4.2.2. Sea $\mathbb{F}$ el conjunto de todas las funciones $f: S \rightarrow$ A para las que $f(x) \in A(s)$ para todo $s \in S$. Se llamarán políticas deterministas estacionarias a las políticas para las cuales existe una función $f \in \mathbb{F}$ tal que $\pi_{n}\left(\cdot \mid h_{n}\right)$ está concentrada en $f\left(x_{n}\right) \in A(s)$ para toda $h_{n} \in H_{n} y$ $n=0,1, \ldots$ 
El conjunto de todas las políticas se denotará con $\mathbb{P}$ y al conjunto de las deterministas con $\mathbb{F}$, es claro que $\mathbb{F} \subset \mathbb{P}$.

Con las definiciones anteriores, ahora se tienen todas las condiciones para definir a lo que llamaremos el proceso de control de Markov.

Dada una política $\pi$ y un estado inicial $x_{0}$, queda determinado un proceso estocástico cuya dinámica se puede describir como sigue a continuación:

Supóngase que en el tiempo $n$ el proceso tiene una historia $h_{n}$ y además se está en el estado $x_{n}$, en ese instante se elige una acción(de alguna manera) de acuerdo a $\pi$. Sea $a_{n}$ la acción dictada por $\pi$, entonces el proceso se moverá al estado $y$ con probabilidad $Q\left(\{y\} \mid x_{n}, a_{n}\right)$. Esta dinámica junto con la política $\pi$ y un estado inicial $s$ definen todas las distribuciones finito-dimensionales $x_{0}, a_{0}, \ldots, x_{n-1}, a_{n-1}, \xi_{n}, n \in \mathbb{N}$; el Teorema de Io-

nescu Tulcea [2] garantiza que dados $x$ y $\pi$ la definición de las siguientes sucesiones $\left\{X_{n}\right\},\left\{A_{n}\right\}$ de estados y controles respectivamente existen.

Con lo anterior se puede denotar a la probabilidad y a la esperanza relacionandolos con lo anterior por $P_{x}^{\pi}$ y $E_{x}^{\pi}$.

\subsection{Problema de Control Óptimo}

En esta parte se planteará lo que es en general un problema de control de Markov o problema de control óptimo. Considérese una función de la siguiente forma:

$$
V: \mathbb{P} \times S \rightarrow \mathbb{R}
$$

la cual medirá el resultado que se obtiene a lo largo del proceso, bajo las acciones dictadas por alguna política $\pi$, dado que el estado inicial fue 
un $s$ fijo. A una función que cumpla con lo anterior la llamaremos función objetivo. Lo que se puede observar es que en general esta función estará relacionada con la función de respuesta por etapa.

Definición 4.3.1. Dados un $M C M\{(S, A,\{A(s) \mid s \in S\}, Q, \rho)\}$ el conjunto de políticas $\mathbb{P}$ y una función objetivo $V$. El Problema de Control Óptimo consiste en determinar $\pi^{*} \in \mathbb{P}$ (si es que ésta existe), tal que:

$$
V\left(\pi^{*}, s\right)=\sup _{\pi \in \mathbb{P}} V(\pi, s)
$$

$s \in S$.

Mas aún

Definición 4.3.2. A la función

$$
V(s)=\sup _{\pi \in \mathbb{P}} V(\pi, s)
$$

$s \in S$, le llamaremos función de valor óptimo del PCO

Dado lo anterior de manera natural se sigue lo siguiente.

Definición 4.3.3. Si existe una política $\pi^{*} \in \mathbb{P}$ tal que:

$$
V(s)=V\left(\pi^{*}, s\right)
$$

para todo $s \in S$, entonces a ésta $\pi^{*}$ le llamaremos la política óptima.

El proceso de control de Markov junto con la función objetivo a optimizar es a lo que se conoce como el Problema de Control de Markov (PCM). 


\section{Capítulo 5}

\section{Aplicaciones}

En este capítulo se desarrollarán las aplicaciones de la teoría de matrices no negativas que cumplen con la propiedad producto, con respecto a la recursión planteada en (3.1) para una matriz de Leontief de $2 \times 2$ y en el caso neutral y sensible al riesgo de un problema de control estocástico. En donde para la recursión (3.1), el primero ilustra un caso irreducible y los otros, el caso reducible. Cabe señalar que, por simplicidad, para el ejemplo de la matriz de Leontief se muestra para un modelo de dos empresas, sin embargo se podría generalizar para un número mayor de éstas.

\subsection{Modelo de Leontief}

El modelo de Input-Output propuesto por Wassily Leontief, el cual provee un método sistemático para el análisis de las interrelaciones entre las industrias y sus transacciones, le permitió en el año de 1973 ganar el premio nobel de economía.

A grandes rasgos el modelo supone que la economía está conformada por diversos sectores de producción y servicios. En donde por un lado existe una interrelación interna que satisfacer entre los sectores y al mismo 
tiempo, por otro lado existe una demanda externa.

El análisis de los cuadros de insumo-producto, fue desarrollado como un instrumento de interpretación de las interdependencias de los diversos sectores de la economía. Es decir, en estos, se supone cualquier sistema económico como un complejo de industrias mutuamente interrelacionadas. Se considera además, que toda industria recibe materias primas (insumos) de las demás industrias del sistema y que a su vez, proporciona su producción a las demás industrias en calidad de materia prima.

Fundamentalmente se trata de un análisis general del equilibrio estático de las condiciones tecnológicas de la producción total de una economía, durante el periodo de tiempo en cuestión.

Cabe hacer notar, que el hecho de estimar la matriz de insumoproducto puede ser un arduo trabajo, pero para esta parte de la tesis se supondrá que la matriz Q será dada de algún modo y que cumple con que es una matriz subestocástica(definición en las siguientes lineas), la cual se piensa estática. Sea:

$$
Q=\left(\begin{array}{ll}
q_{11} & q_{12} \\
q_{21} & q_{22}
\end{array}\right)
$$

la matriz que representa el consumo; obsérvese que Q es una matriz subestocástica es decir:

(a) $q_{i j} \geq 0$;

(b) $q_{11}+q_{12} \leq 1 ; \mathrm{y}$

(c) $q_{11}+q_{12} \leq 1$.

Como se puede observar en caso de que se quisiera utilizar la teoría de los proceso de Markov, se debería recurrir a generar una forma para la cual, la matriz anterior $Q$ sea transformada en una matriz $\tilde{Q}$ de tal manera 
que $\tilde{Q}$ sea estocástica. Así que para solucionar este pequeño problema lo que se puede hacer es introducir una nueva columna de la siguiente forma:

$$
\tilde{Q}:=\left(\begin{array}{ccc}
q_{11} & q_{12} & 1-q_{11}-q_{12} \\
q_{21} & q_{22} & 1-q_{21}-q_{22} \\
0 & 0 & 1
\end{array}\right) .
$$

Obsérvese que el paso anterior soluciona el problema y a partir de aquí se puede hacer todo un análisis dado que se conocen los valores de $q_{i i}$ para toda $i$, una vez que ya se tiene lo precedente se esta en condiciones de utilizar toda la herramienta matemática para describir el comportamiento al límite. Otra opción que también es viable, es tomar la matriz subestócastica y analizarla. Por ejemplo, supóngase que se tiene la siguiente matriz de insumo producto:

$$
Q:=\left(\begin{array}{ll}
.4 & .3 \\
.2 & .4
\end{array}\right),
$$

y sea $\mathrm{P}$ la matriz extendida.

$$
P:=\left(\begin{array}{ccc}
.4 & .3 & .3 \\
.2 & .4 & .4 \\
0 & 0 & 1
\end{array}\right)
$$

Para este ejemplo se tienen dos fábricas A, B y un estado adicional que en el ambiente económico se le conoce como el banco. Aún más, se está suponiendo que las fábricas guardan su dinero en el mismo banco. Entonces los números $q_{i j}$ representan la cantidad de producción de la fábrica $i$ que la fábrica $j$ necesita por cada dólar de producción. Es decir los números de la primera fila representan que por cada dolar de producto de A, la fábrica A necesita: 
.4 de producto de $\mathrm{A}$;

.3 de producto de $\mathrm{B}$;

.3 guarda en el banco.

Como se puede ver el banco es un estado absorbente, por lo que en el límite se tiene que todo el dinero llegará al banco.

Lo anterior promueve que se planteen otro tipo de preguntas, por ejemplo, si se piensa el dinero como una pila de billetes(monedas) y se define $X_{n}=$ como el lugar que ocupará un billete aleatorio al tiempo n y se supone que se tienen 100 billetes y que de ellos se ponen 50 en el banco, 30 se gastan en el producto de A y 20 en el producto B. Dada la condición anterior se tiene que $X_{0}=(.3, .2, .5)$. Si se marca un billete se tiene que la probabilidad de que vaya al banco es .5, que vaya a la fábrica $\mathrm{A}$ es .3 y que vaya a la fábrica B es .2. También supóngase que cada fábrica mantiene un cierto stock de suplementos, lo que significa que para el siguiente período una cierta cantidad del stock será usado y así el dinero se habrá movido del estado 0 al estado 1 , es decir, se tiene que $X_{1}=X_{0} * Q$ por lo cual se obtiene que: $X_{1}=(0.26,0.32,0.42)$. En particular en este ejemplo se desarrollará la entrada que representa el banco; en la que en el período $t=1$ se cuenta con 42 billetes. Esto se puede observar como: La fábrica A pone el $30 \%$ en el banco, así que se tiene: 40*.3=12 y para el caso de la fábrica B es $50^{*} .4=20$ sumando $12+20+10=42$, es el resultado que se representa en el banco pero en porcentaje. Por lo que para cualquier tiempo $n$ se puede hacer un análisis análogo al anterior: de donde siguiendo el proceso se tiene que $X_{n}=X_{0} * P^{n}$ lo que representaría la distribución del dinero después de $n$ días.

Ahora si se utiliza la teoría de los procesos de Markov, la siguiente pregunta que surge es ¿cuánto tiene que producir la empresa A en total?

Para responder a la cuestión planteada, obsérvese lo siguiente: $100\left(X_{0}+\right.$ 
$\left.X_{0} P+X_{0} P^{2}+\ldots\right)$, algo interesante es que para este caso se puede dejar de lado la entrada que representa al banco ya que esto no afecta en la producción.

Si se toma una condición inicial dada por $(30,40)$ entonces se tiene que: $(30,40)\left(I+Q+Q^{2}+Q^{3}+\ldots\right)$ y se puede observar que esta serie converge a $(I-Q)^{-1}$. Lo cual para este caso, dado que el $\operatorname{det}(I-Q)^{-1} \neq 0$ entonces se puede calcular y esta dada por:

$$
(I-Q)^{-1}=\left(\begin{array}{cc}
2 & 1 \\
.6667 & 2
\end{array}\right),
$$

entonces $(30,40)(I-Q)^{-1}=(\$ 86.6667, \$ 110)$

Así que se obtiene que la empresa A debe de producir $\$ 86.6667$ en total y lo anterior se puede hacer con cada entrada. Cabe resaltar que en [28] se puede encontrar toda una descripción sobre algunas caracterizaciones para saber cuando $(I-Q)^{-1}$ existe.

Lo precedente demuestra una parte del análisis que se puede hacer a la matriz $Q$, pero la idea es ir un poco más allá y utilizar para este caso en particular la técnica de matrices no negativas, la cual permite aproximarnos a la solución mediante el uso de cotas. Una vez más retómese el ejemplo anterior y recupérese la ecuación:

$X_{n}=X_{0} * Q^{n}$ la cual acorde con la notación usada durante este trabajo es: $x(n)=x(0) * Q^{n}$

Dado lo anterior, obsérvese que $Q$ es una matriz irreducible ya que sólo tiene una clase de comunicación entonces por la técnica de matrices no negativas, se tiene que la relación se cumple:

$$
c_{1} \sigma^{n} \tilde{u} \leq x(n) \leq c_{2} \sigma^{n} \tilde{u}
$$

en donde $c_{1}$ y $c_{2}$ son constantes que dependen de que

$$
c_{1} \tilde{u} \leq x(0) \leq c_{2} \tilde{u}
$$


Para este caso específico se sigue que:

(a) $\sigma_{\text {esp }}=.7$;

(b) $\tilde{u}=(.7809, .6447)$;

(c) $X_{0}=(.3, .2)$.

Así que tomando $c_{1}=1 / 3$ y $c_{2}=1 / 2$ se concluye inmediatamente que: $x(n) \leq(.5)(.7)^{n}(.7809, .6447)$ y además es claro que cuando $n$ crece el lado derecho tiende a cero lo que nos indica que $x(n)$ tiende a cero, es decir, que la tendencia de la economía los lleva a dejar el dinero afuera de la reinversión; en el caso en el que se anexa el estado del banco nos dice que el dinero se va al banco. Esto es sencillo de ver ya que cuando se trabaja con la matriz $\mathrm{P}$ el único estado absorbente es el banco. Así que por la teoría de los procesos de Markov se tiene que

$$
\lim _{n \rightarrow \infty} P^{n}:=\left(\begin{array}{lll}
0 & 0 & 1 \\
0 & 0 & 1 \\
0 & 0 & 1
\end{array}\right) .
$$

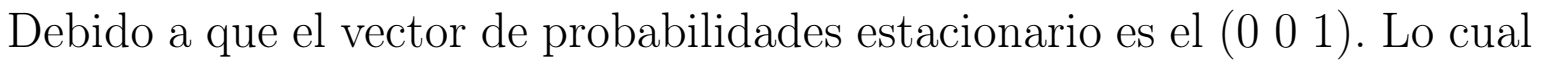
es otra manera de aproximación de lo anterior.

Como se puede notar para un mismo problema existen diferentes formas de atacarlo y con esto obtener resultados interesantes que permiten dar diferentes interpretaciones a los fenómenos que ocurren a nuestro alrededor, ya sea en la idea del promedio o en la idea de los comportamientos al límite. Es importante recalcar que es justo esta idea la del límite la que permite en muchos ámbitos hacer suposiciones sobre los comportamientos. 


\subsection{Caso Neutral al Riesgo: un Juego de Apues- tas}

Considérese un modelo de control semejante al presentado en (4.1)

$$
(S, A,\{A(s) \mid s \in S\}, Q, R)
$$

en donde tanto como $S$ y $A$ son finitos, además para este apartado se está usando $R$ para denotar la función de respuesta $\rho$, debido a que para el caso que se trabajará la función $R$ será relacionada con la recompensa, más aún ésta la supondremos no negativa.

La función objetivo del Proceso de Control de Markov (PCM) será la esperanza de la recompensa total; es decir dado el modelo de control y el conjunto de políticas asociadas a él, considérese la siguiente función objetivo para una política $\pi \in \mathbb{P}$ y un estado inicial $s_{0}=s \in S$.

$$
E_{s}^{\pi}\left[\sum_{n=0}^{\infty} R\left(X_{n}, A_{n}\right)\right],
$$

$s \in S$. Aunque esta medida del funcionamiento de una política no necesariamente es apropiada, para este trabajo será la que se utilizará para el modelo que se propondrá en el ejemplo.

Se denotará por $V(\pi, s)$ a la Esperanza de la recompensa total ganada bajo la política $\pi$ cuando el estado inicial es $s_{0}=s$, es decir

$$
V(\pi, s)=E_{s}^{\pi}\left[\sum_{n=0}^{\infty} R\left(X_{n}, A_{n}\right)\right],
$$

$s \in S$. Para este caso se dirá que se tiene un problema con horizonte infinito. Ademas como $R \geq 0, V(\pi, s)$ está bien definida, aunque se podría dar el caso en el que este valor fuera infinito. Por lo que la función de valor 
óptimo 4.8 para este caso se transforma en:

$$
V(s)=\sup _{\pi \in \mathbb{P}} E_{s}^{\pi}\left[\sum_{n=0}^{\infty} R\left(X_{n}, A_{n}\right)\right],
$$

para todo $s \in S$ y se tendrá que $\pi^{*}$ es óptima si se cumple que:

$$
V(s)=V\left(\pi^{*}, s\right)
$$

para todo $s \in S$

\subsubsection{Planteamiento del Problema}

\section{Un Juego de Apuestas propuesto por Ross}

A continuación se presenta el juego que propuso Ross pág.76 [21], el cual provee la aplicación de esta sección.

Considérese la siguiente situación. Un individuo tiene $i$ dólares para jugar en un casino. El casino permite cualquier apuesta si se siguen las siguientes reglas: Si se poseen $i$ dólares entonces se puede apostar cualquier cantidad menor o igual a $i$. Más aún si el individuo apuesta $j$ entonces:

- Se ganan $j$ con probabilidad $p$ ó

- Se pierden $j$ con probabilidad $1-p$

La pregunta que surge es ¿Cuál es la estrategia que maximiza la probabilidad de que el jugador alcance la fortuna $N$ antes de irse a la quiebra? Sea $\{0,1,2, \ldots, N\}$ el espacio de estados, se dice que el estado es $i$ cuando se tiene como fortuna $i$. Se define la estructura de la recompensa por:

$$
R(i, a)=0
$$


$i \neq N$ para toda $a$

$$
R(N, a)=1
$$

y se tiene que:

$$
P_{N, 0}(a)=P_{0,0}(a)=1 \text {. }
$$

En otras palabras lo que explica lo anterior es que se obtiene como recompensa $\$ 1$ si y sólo si se alcanza la fortuna $N$; con esto se tiene que la recompensa total esperada es justo la probabilidad de que la fortuna del jugador sea $N$.

Para determinar la política óptima lo primero que se debe notar es que si la fortuna presente es $i$ entonces nunca se puede apostar más que $N-i$. Esto significa que dado que se tiene la fortuna $i$ las posibles elecciones que se tienen para apostar es $1,2, \ldots, \operatorname{mín}(i, N-i)$.

Si se utiliza el resultado sobre la desigualdad de optimalidad, entonces se busca una política $U$ de tal forma que:

$$
U(i) \geq p U(i+k)+(1-p) U(i-k)
$$

para todo $0<i<N$ y $k \leq \operatorname{mín}(i, N-i)$ y entonces la política que se eligiría sería óptima.

Considérese el siguiente modelo de decisión de Markov.

\section{Modelo}

Para un entero positivo fijo $N$ y un número $p \in[0,1]$ los cincos elementos del modelo quedaran definidos como sigue a continuación:

(a) $S:=[0,1,2, \ldots, N]$ el espacio de estados del modelo

(b) $A:=\{0,1,2, \ldots,[N / 2]\}$ el espacio de acciones o controles, en donde $[z]$ representa la parte entera de $z$.

(c) $A(s):=$ Para cada $s \in S, A(s)=\{1,2, \ldots, \operatorname{mín}\{s, N-s\}\}$ 
(d) Se define la siguiente ley de transición $Q=q_{s y}(a)$ para $s \in S$ y $a \in A(s)$ como :

1. $q_{s, s+a}(a)=p$

2. $q_{s, s-a}(a)=q$ donde $q=1-p$

3. $q_{N, 0}(a)=1$

4. $q_{0,0}(a)=1$

(e) La función de respuesta $(\rho)$ en este caso de recompensa por etapa como

$$
R(s . a)=0, s \neq N ; R(N, a)=1 .
$$

Para este modelo se define la función objetivo $V$ como:

$$
V(\pi, s)=E_{s}^{\pi}\left(\sum_{n=0}^{\infty} R\left(X_{s}, A_{s}\right)\right.
$$

Una vez que se tiene lo anterior, se utilizará la técnica de matrices no negativas para resolver este problema, el cual se explica de la siguiente forma:

Como el problema se desarrolla con un espacio de estados y acciones finitos; se considerará el tiempo de forma discreta. Se tiene que para cada período de tiempo el sistema sólo puede estar en un número finito de estados, llamémosle $1,2, \ldots N$. Si al tiempo $n$, el sistema está en el estado $i$, entonces se puede elegir una acción $a$ del espacio de estados de acciones admisibles para $i$. Esta acción, da como resultado una probabilidad $q_{i, j}^{a}$ de que el sistema se encuentre en $j$ en el tiempo $t+1$. Más aún dado lo anterior se obtiene una recompensa $r_{i j}^{a}$ por haber tomado la acción $a$ cuando se estaba en el estado $i$. Supóngase que: 


$$
r_{i j}^{a} \geq 0 ; \quad \sum_{j=1}^{n} q_{i j}^{a} \leq 1
$$

$i, j=1, \ldots, N ; a \in A$ i.e. existe una probabilidad positiva de que el proceso termine.

Sea $v(0)_{i}$ que denota la recompensa en el estado $i$ y sea $v(n)_{i}$ que denota la máxima recompensa esperada para el problema en el n-ésimo período. (i.e con $n$ períodos por suceder), dado que se comienza en el estado $i$. Se define la recompensa

$$
r_{i}^{a}=\sum_{j=1}^{N} q_{i j}^{a} r_{i j}^{a}
$$

$i=1, \ldots N ; a \in A$. El principio de Optimalidad de Bellman's implica que la siguiente recursión para $v(n)_{i}$ es válida.

$$
v(n)_{i}=\operatorname{máx}_{a \in A}\left\{r_{i}^{a}+\sum_{j=1}^{N} q_{i j}^{a} v(n-1)_{j}\right\}
$$

La ecuación anterior puede ser escrita con notación vectorial cuando se introduce la terminología de política como se había visto anteriormente. Sea $\mathrm{P}(\pi)$ que denota la matriz con entradas $q_{i j}^{\pi(i)}$ y $r(\pi)$ el vector con componentes $r_{i}^{\pi(i)}$ para $i, j=1,2, \ldots N ; \pi \in \mathbb{P}$. Con lo anterior se tiene que la colección de $N \times(N+1)$ matrices $\{(P(\pi), r(\pi) \mid \pi \in \mathbb{P})\}$ tiene la propiedad producto. Así que en vez de utilizar la ecuación anterior, para este instante se puede manejar la siguiente ecuación:

$$
v(n)=\operatorname{máx}_{\pi \in \mathbb{P}}\{r(\pi)+P(\pi) v(n-1)\},
$$

$n \in \mathbb{N}$, donde $v(n)$ denota el vector con componentes $v(n)_{i}, i=1,2, \ldots N$; con la introducción de una simple constante se obtiene que:

$$
\left(\begin{array}{c}
v(n) \\
1
\end{array}\right)=\operatorname{máx}_{\pi \in \mathbb{P}}\left(\begin{array}{cc}
P(\pi) & r(\pi) \\
0 & 1
\end{array}\right)\left(\begin{array}{c}
v(n-1) \\
1
\end{array}\right)
$$


$n \in \mathbb{N}$. Lo anterior permite de manera general observar que es lo que se debe hacer para resolver el problema planteado anteriormente.

Lo que sigue a continuación es utilizar lo que se ha ido trabajando pero para este ejemplo. Esto se hará paso a paso, primero describiendo que sucede para cada elemento del problema de control.

Ejemplo 5. Sea $S:=\{0,1,2,3,4\}$, el espacio de estados; entonces el espacio de acciones disponibles queda dado por: $A(0)=\{0\}, A(1)=$ $\{1\}, A(2)=\{1,2\}, A(3)=\{1\}, A(4)=\{0\}$ y sean $Q$ y $R$ definidas como al principio de esta sección. Entonces la técnica de matrices no negativa, explica que para cada política $\pi$ tenemos una matriz asociada $P(\pi)$ y un vector de recompensa $R(\pi)$; el siguiente paso es generar una nueva matriz $\overline{P^{\pi}}$ para cada política posible, la cual ya contiene el nuevo estado; es en este paso en donde se utilizan varios resultados importantes, aunque no parece muy claro, se esta utilizando una propiedad que permite hablar de cerradura del espacio de matrices con las que se está trabajando, las cuales cumplen la propiedad producto (esto debido a que al utilizar las políticas deterministas, sólo se considera las posibles combinaciones que existen respecto al espacio de acciones), más aún el radio espectral $\sigma\left(P^{\pi}\right)=1$ para toda $\pi \in P$, además de que las clases básicas de este ejemplo son exactamente el estado inicial y el final por lo que el grado de $\mathrm{P}$ que es igual a $\nu=1$. Dado lo anterior se está en condiciones para comenzar el proceso iterativo y encontrar la solución al problema cuando $S:=\{0,1,2,3,4\}$.

Una vez que se tiene el número de estados y el conjunto de acciones disponibles para cada estado $s \in S$ hay que determinar el número de políticas que se tienen para este problema. Una forma gráfica de observar esto es mediante la creación de árboles sobre los posibles valores que toma 
$s$ en el espacio de acciones.

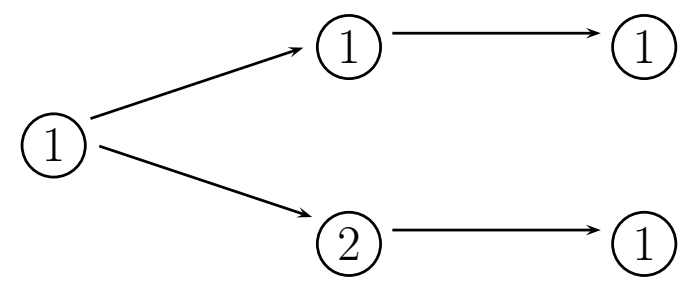

En particular para este caso se tienen dos posibles políticas. $\pi_{1}=$ $(1,1,1)$ y $\pi_{2}=(1,2,1)$. Lo que se quiere es determinar cuando el valor esperado respecto a la política $\pi_{1}>\pi_{2}$, cabe notar que hasta ahora, hemos olvidado un poco de que las matrices de transición depende de p y q así que se pueden tener dos casos cuando $p \geq q$ y cuando $q>p$. El objetivo es encontrar la política óptima.

Para hacer más claro lo anterior, obsérvese lo siguiente:

$$
\pi_{1}:=\left(\begin{array}{cccccc}
1 & 0 & 0 & 0 & 0 & 0 \\
q & 0 & p & 0 & 0 & 0 \\
0 & q & 0 & p & 0 & 0 \\
0 & 0 & q & 0 & p & 0 \\
1 & 0 & 0 & 0 & 0 & 1 \\
0 & 0 & 0 & 0 & 0 & 1
\end{array}\right) \quad \pi_{2}:=\left(\begin{array}{cccccc}
1 & 0 & 0 & 0 & 0 & 0 \\
q & 0 & p & 0 & 0 & 0 \\
q & 0 & 0 & 0 & p & 0 \\
0 & 0 & q & 0 & p & 0 \\
1 & 0 & 0 & 0 & 0 & 1 \\
0 & 0 & 0 & 0 & 0 & 1
\end{array}\right)
$$

Las matrices precedentes representan a la política $\pi_{1}$ y $\pi_{2}$ ya con el vector de recompensa y la extensión que se necesitaba para que la matriz fuera cuadrada. Una vez que se tiene lo anterior, se está en condiciones para proceder a seleccionar cuál de las dos valores esperados de las políticas es mejor dado que se tiene un valor de $p$.

Supóngase que $p=1 / 2$ entonces si se utiliza el algoritmo que se describirá más adelante, resulta que tanto la política $\pi_{1}$ como $\pi_{2}$ son óptimas y que el 
valor óptimo $v$ es: $[0,0.7529,0.9412,0.9882]$. El cual resulta de maximizar la probabilidad de llegar a $N$ antes que a 0 . (Este resultado sobre maximizar la probabilidad se encuentra en [1], el cual se fundamenta en la desigualdad de optimalidad)

\subsubsection{Caso Algoritmo General}

Hemos de hacer hincapié que para el resultado general otra vez se está utilizando que tanto el grado de $\left(P^{\pi}\right)$, así como el radio espectral son iguales a 1. $(\nu=\sigma=1)$. Lo anterior es fácil de probar debido que los únicos estados básicos son el inicial y el final. Además de que por la definición del problema a resolver el radio espectral es igual a 1; la demostración de la convergencia al valor óptimo se tiene utilizando los resultados provistos por la técnica de matrices no negativas, Un hecho importante es que las matrices que se tienen son reducibles, por lo que tienen una intrínseca relacion con las cadenas de Markov absorbentes. En [16], hay todo un tratado sobre las cadenas de Markov absorbentes, para las cuales se puede calcular la matriz fundamental y calcular el tiempo promedio de absorción, el cual cuando la matriz es finita el tiempo promedio de absorción también es finito. Así que este hecho, aunado a que el espacio de estados para este ejemplo es finito se tiene que el valor puede ser estimado en un número finito de pasos.

Lo primero que hay que notar es que el número de posibles políticas depende de $\mathrm{N}$ (números de estados antes de la extensión); Sea $\bar{N}=N-1$ es decir $\bar{N}$ representa a los estados intermedios entre 0 y $\mathrm{N}$.

Proposición 5.2.1. Si el número $\bar{N}>2$ es par entonces el número de políticas es igual $((\bar{N} / 2) !)^{2}$ y si $\bar{N}>2$ es impar entonces el número de políticas es igual $((\bar{N}+1) / 2) ! \times((\bar{N}-1) / 2) !$ 
Demostración: Obsérvese el caso para $\bar{N}=3$ entonces las posibles posibilidades para los estados intermedios son $(1,2,1)$, en donde cada dígito representa una acción, por lo que el número 2 representa las acciones $(1,2)$, para este caso se tiene que el número posible de políticas es $1 \times 2 \times 1=2$ lo cual es equivalente a $((\bar{N}+1) / 2) ! \times((\bar{N}-1) / 2)$ !. Obsérvese que para el caso $\mathrm{N}=3$ la fórmula se transforma en $2 ! \times 1 !=2$ por lo que se cumple. Si se toma el caso para el cual $\bar{N}=4$, se tiene que las posibilidades para cada estado son $(1,2,2,1)$ de donde se tiene que $1 \times 2 \times 2 \times 1=4$ que es igual $((\bar{N} / 2) !)^{2}=4$.

Supóngase válido para k fija impar entonces se tienen dos casos:

Cuando k es impar se cumple que:

$(1,2,3, \ldots,(k-3) / 2,(k-1) / 2,(k+1) / 2,(k-1) / 2,(k-3) / 2, \ldots, 3,2,1)=$ $((k+1) / 2) ! \times((k-1) / 2) !$

Cuando $\mathrm{k}+1$ es par se cumple que:

$(1,2,3, \ldots((k+1) / 2)-2,((k+1) / 2)-1,(k+1) / 2,(k+1) / 2,((k+1) / 2)-$ $1,((k+1) / 2)-2 \ldots, 3,2,1)=((k+1) / 2) !)^{2}$

Lo anterior se hace por la diferencia que existe entre los casos, sea $k+2=k^{*}$ impar, se tienen dos casos:

Cuando $k^{*}$ es impar entonces tenemos

$\left(1,2,3, \ldots,\left(k^{*}-1\right) / 2,\left(k^{*}+1\right) / 2,\left(k^{*}-1\right) / 2, \ldots, 3,2,1\right)$, obsérvese que haciendo una reordenación se tiene que:

$\left(1,2,3, \ldots,\left(k^{*}-1\right) / 2, \ldots, 3,2,1,\left(k^{*}+1\right) / 2,\left(k^{*}-1\right) / 2\right)^{P . H . I}((k+1) / 2) ! \times$ $((k-1) / 2) ! \times\left(k^{*}+1\right) / 2 \times\left(k^{*}-1\right)=\left(\left(k^{*}+1\right) / 2\right) ! \times\left(\left(k^{*}-1\right) / 2\right) !$ por lo tanto se cumple para $k^{*}+1$.

Ahora para el caso $k^{*}+1$ que es par:

$\left(1,2,3, \ldots,\left(\left(k^{*}\right) / 2\right)-1,\left(k^{*}+1\right) / 2,\left(k^{*}+1\right) / 2,\left(\left(k^{*}\right) / 2\right)-1, \ldots, 3,2,1\right)$ y para este caso igual solo se hace una reordenación y se tiene que: 
$\left(1,2,3, \times,\left(k^{*}-1\right) / 2,\left(k^{*}-1\right) / 2, \ldots, 3,2,1,\left(k^{*}+1\right) / 2,\left(k^{*}+1\right) / 2\right)^{\text {P.H.I }}=\left(\left(k^{*}-\right.\right.$ $1) / 2) !\left(\left(k^{*}-1\right) /\right) 2 ! \times\left(\left(k^{*}+1\right) / 2\right) ! \times\left(\left(k^{*}+1\right) / 2\right) !=\left(\left(\left(k^{*}+1\right) / 2\right) !\right)^{2}$ de donde se cumple para $k^{*}+1$.

El código "Algoritmo neutral al riesgo" permite solucionar el problema solo dándole el número de estados intermedios +1 , p y M(el número de horizonte de pronóstico) está dado mediante una cota.

A continuación se presentan los resultados obtenidos. 


\begin{tabular}{|c|c|c|c|c|c|c|c|c|c|}
\hline $\mathrm{P}$ & $Q$ & $\# \bar{N}$ & 3 & 4 & 5 & 6 & 7 & 8 & 9 \\
\hline .1 & .9 & P.O & $(1,2,1)$ & $(1,2,2,1)$ & $\begin{array}{l}(1,1,3,1,1) \\
(1,1,3,2,1) \\
(1,2,3,1,1) \\
(1,2,3,2,1)\end{array}$ & $(1,2,3,3,2,1)$ & $\begin{array}{l}(1,2,1,4,1,2,1) \\
(1,2,1,4,3,2,1) \\
(1,2,3,4,1,2,1) \\
(1,2,3,4,3,2,1)\end{array}$ & $(1,2,3,4,4,3,2,1)$ & $\begin{array}{l}(1,2,2,1,5,1,2,2,1) \\
(1,2,2,1,5,1,3,2,1) \\
(1,2,2,1,5,4,2,2,1) \\
(1,2,2,1,5,4,3,2,1) \\
(1,2,2,4,5,1,2,2,1) \\
(1,2,2,4,5,1,3,2,1) \\
(1,2,2,4,5,4,2,2,1) \\
(1,2,2,4,5,4,3,2,1) \\
(1,2,3,1,5,1,2,2,1) \\
(1,2,3,1,5,1,3,2,1) \\
(1,2,3,1,5,4,2,2,1) \\
(1,2,3,1,5,4,3,2,1) \\
(1,2,3,4,5,1,2,2,1) \\
(1,2,3,4,5,1,3,2,1) \\
(1,2,3,4,5,4,2,2,1) \\
(1,2,3,4,5,4,3,2,1)\end{array}$ \\
\hline .2 & .8 & P.O. & $(1,2,1)$ & $(1,2,2,1)$ & $\begin{array}{l}(1,1,3,1,1) \\
(1,1,3,2,1) \\
(1,2,3,1,1) \\
(1,2,3,2,1)\end{array}$ & $(1,2,3,3,2,1)$ & $\begin{array}{l}(1,2,1,4,1,2,1) \\
(1,2,1,4,3,2,1) \\
(1,2,3,4,1,2,1) \\
(1,2,3,4,3,2,1)\end{array}$ & $(1,2,3,4,4,3,2,1)$ & $\begin{array}{l}(1,2,2,1,5,1,2,2,1) \\
(1,2,2,1,5,1,3,2,1) \\
(1,2,2,1,5,4,2,2,1) \\
(1,2,2,1,5,4,3,2,1) \\
(1,2,2,4,5,1,2,2,1) \\
(1,2,2,4,5,1,3,2,1) \\
(1,2,2,4,5,4,2,2,1) \\
(1,2,2,4,5,4,3,2,1) \\
(1,2,3,1,5,1,2,2,1) \\
(1,2,3,1,5,1,3,2,1) \\
(1,2,3,1,5,4,2,2,1) \\
(1,2,3,1,5,4,3,2,1) \\
(1,2,3,4,5,1,2,2,1) \\
(1,2,3,4,5,1,3,2,1) \\
(1,2,3,4,5,4,2,2,1) \\
(1,2,3,4,5,4,3,2,1)\end{array}$ \\
\hline .3 & .7 & P.O & $(1,2,1)$ & $(1,2,2,1)$ & $\begin{array}{l}(1,1,3,1,1) \\
(1,1,3,2,1) \\
(1,2,3,1,1) \\
(1,2,3,2,1)\end{array}$ & $(1,2,3,3,2,1)$ & $\begin{array}{l}(1,2,1,4,1,2,1) \\
(1,2,1,4,3,2,1) \\
(1,2,3,4,1,2,1) \\
(1,2,3,4,3,2,1)\end{array}$ & $(1,2,3,4,4,3,2,1)$ & $\begin{array}{l}(1,2,2,1,5,4,2,2,1) \\
(1,2,2,1,5,4,3,2,1) \\
(1,2,2,4,5,4,2,2,1) \\
(1,2,2,4,5,4,3,2,1) \\
(1,2,3,1,5,4,2,2,1) \\
(1,2,3,1,5,4,3,2,1) \\
(1,2,3,4,5,4,2,2,1) \\
(1,2,3,4,5,4,3,2,1)\end{array}$ \\
\hline
\end{tabular}




\begin{tabular}{|c|c|c|c|c|c|c|c|c|c|}
\hline .4 & .6 & P.O & $(1,2,1)$ & $(1,2,2,1)$ & $\begin{array}{l}(1,1,3,1,1) \\
(1,1,3,2,1) \\
(1,2,3,1,1) \\
(1,2,3,2,1)\end{array}$ & $(1,2,3,3,2,1)$ & $\begin{array}{l}(1,2,1,4,1,2,1) \\
(1,2,1,4,3,2,1) \\
(1,2,3,4,1,2,1) \\
(1,2,3,4,3,2,1)\end{array}$ & $(1,2,3,4,4,3,2,1)$ & $\begin{array}{l}(1,2,2,1,5,1,2,2,1) \\
(1,2,2,1,5,1,3,2,1) \\
(1,2,2,1,5,4,2,2,1) \\
(1,2,2,1,5,4,3,2,1) \\
(1,2,2,4,5,1,2,2,1) \\
(1,2,2,4,5,1,3,2,1) \\
(1,2,2,4,5,4,2,2,1) \\
(1,2,2,4,5,4,3,2,1) \\
(1,2,3,1,5,1,2,2,1) \\
(1,2,3,1,5,1,3,2,1) \\
(1,2,3,1,5,4,2,2,1) \\
(1,2,3,1,5,4,3,2,1) \\
(1,2,3,4,5,1,2,2,1) \\
(1,2,3,4,5,1,3,2,1) \\
(1,2,3,4,5,4,2,2,1) \\
(1,2,3,4,5,4,3,2,1)\end{array}$ \\
\hline .5 & .5 & $\mathrm{P} . \mathrm{O}$ & $\pi_{i}$ & $\pi_{i}$ & $\pi_{i}$ & $\pi_{i}$ & $\pi_{i}$ & $\pi_{i}$ & $\pi_{i}$ \\
\hline .6 & .4 & P.O & $\pi_{1}$ & $\pi_{1}$ & $\pi_{1}$ & $\pi_{1}$ & $\pi_{1}$ & $\pi_{1}$ & $\pi_{1}$ \\
\hline .7 & .3 & $\mathrm{P} . \mathrm{O}$ & $\pi_{1}$ & $\pi_{1}$ & $\pi_{1}$ & $\pi_{1}$ & $\pi_{1}$ & $\pi_{1}$ & $\pi_{1}$ \\
\hline .8 & .2 & $\mathrm{P} . \mathrm{O}$ & $\pi_{1}$ & $\pi_{1}$ & $\pi_{1}$ & $\pi_{1}$ & $\pi_{1}$ & $\pi_{1}$ & $\pi_{1}$ \\
\hline .9 & .1 & $\mathrm{P} . \mathrm{O}$ & $\pi_{1}$ & $\pi_{1}$ & $\pi_{1}$ & $\pi_{1}$ & $\pi_{1}$ & $\pi_{1}$ & $\pi_{1}$ \\
\hline
\end{tabular}

En donde $\pi_{1}$ denota la política en la que siempre se avanza de uno en uno. $(1, \ldots, 1)$, además es claro que cuando $p>q$ la política óptima es la anterior que es conocida en el ambiente probabilístico como la tímida; cabe notar que cuando $q>p$ hay una política la cual aparece en todos los casos y esta es la conocida como la audaz; esta política elige una acción más agresiva, pues cuando el sistema está en un estado $s \leq N / 2$, se elige la acción que le permitiría avanzar el doble del estado actual y cuando el sistema está en un $s \geq N / 2$, su elección es todo lo necesario para que de avanzar, se llegue al objetivo $N$. Nota: Para el caso cuando $p=1 / 2, \pi_{i}$ denota que para cualquier $i$, la selección que se elige es óptima.

Así que se pueden resumir los resultados como: 


\section{Si $p>q$ entonces la política óptima es la tímida y es única Si $q>$ p entonces la política óptima es la audaz y para algunos casos especiales se tiene que esta no es única Si $q=p$ entonces cualquier política que se elige es óptima}

\subsubsection{Cota de Pronóstico}

La cota de pronóstico es una estimación sobre el número de iteraciones que se necesitan para aproximarse al valor esperado para cada política, para este caso se denotará como $M$ y aparece como $M=N(N+11)$ pero lo que está realmente detrás y que acompaña a la convergencia del algoritmo es que como se puede notar; dada una matriz inicial que representa una política dada, la iteración a la que es sometida tiene como resultado que la información aparezca en el primer y último renglón aunque para ser exactos lo que nos permite recuperar el resultado es que si se toma $\lim _{n \rightarrow \infty}\left(P^{\pi}\right)^{n}=P^{*}$ en donde en $P^{*}$ sólo la primera y la última columna contienen elementos distintos de cero.

Un detalle a resaltar es que la política $\left(\pi_{1}\right)$ en la que se avanza de 1 en 1 domina a las otras en el sentido de que si se toma la diagonal y el acomodo que se tiene de $p$ y de $q$ sobre la matriz entonces al momento de tomar cualquier otra política $\pi_{i}$ diferente, se tiene que para algún estado $i$ ya sea $q$ o $p$ al menos se movieron un estado más en dirección a la primera o la última columna. Por lo que al hacer las iteraciones, que es elevar la matriz a alguna potencia se tiene que al menos para alguna entrada $i, j$ se tiene un cero más respecto a las entradas de la matriz $\pi_{1}$ en la misma iteración por lo que el análisis se reduce al anális de la matriz que representa la política $\pi_{1}$. La cota anterior se propone para que los términos que aparecen en la parte centrales de la matriz iterada, sean tan pequeños 
que su contribución sea nula. Más aún, estos estados son siempre de la forma potencias de $p$ o $q$ respectivamente de la forma $(M+2) / 2, M / 2$ ó $(M-2) / 2$ si $M$ es par ó $(M-1) / 2,(M+1) / 2$ si M es impar.

Obsérvese lo siguiente: Sea $\mathrm{N}=5$ entonces la matriz de representación de la política $\pi_{1}$ se ve de la siguiente forma:

$$
\pi_{1}:=\left(\begin{array}{cccccc}
1 & 0 & 0 & 0 & 0 & 0 \\
q & 0 & p & 0 & 0 & 0 \\
0 & q & 0 & p & 0 & 0 \\
0 & 0 & q & 0 & p & 0 \\
1 & 0 & 0 & 0 & 0 & 1 \\
0 & 0 & 0 & 0 & 0 & 1
\end{array}\right) \cdot \pi_{2}:=\left(\begin{array}{cccccc}
1 & 0 & 0 & 0 & 0 & 0 \\
q & 0 & p & 0 & 0 & 0 \\
q & 0 & 0 & 0 & p & 0 \\
0 & 0 & q & 0 & p & 0 \\
1 & 0 & 0 & 0 & 0 & 1 \\
0 & 0 & 0 & 0 & 0 & 1
\end{array}\right)
$$


Ahora si elevamos $\left(\pi_{1}\right)^{M}$ y $\pi_{2}^{M}$ a la cota de pronóstico se obtiene que:

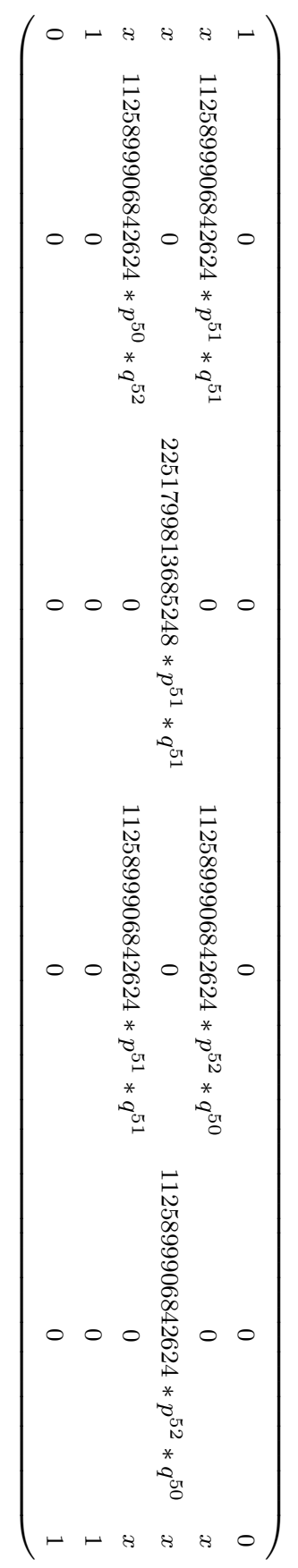




$$
\pi_{2}:=\left(\begin{array}{cccccc}
1 & 0 & 0 & 0 & 0 & 0 \\
x & 0 & 0 & 0 & 0 & x \\
x & 0 & 0 & 0 & 0 & x \\
x & 0 & 0 & 0 & 0 & x \\
1 & 0 & 0 & 0 & 0 & 1 \\
0 & 0 & 0 & 0 & 0 & 1
\end{array}\right)
$$

Cabe notar que las $x$ denotan la información distinta de cero dentro de las matrices. Lo que se puede observar inmediatamente es que los valores que se encuentra en las columnas intermedias tienden a cero cuando la $\mathrm{M}$ crece para el caso $\pi_{1}$; mientras que para el caso $\pi_{2}$ la información intermedia ya ha desaparecido. Más aún dado el resultado que se tiene de que dado un número positivo $\mathrm{M}>1$ entonces el $\lim _{n \rightarrow \infty}\left(\frac{1}{M}\right)^{n}=0$. Éste límite permite decir que el sistema converge y que además a partir de cierta tolerancia se puede acercarse tanto al valor real como sea necesario. Lo anterior se sigue también para cuando el número de estados crece aunque hay que hacer notar que en los estados intermedios aparecen términos tales que la suma de los exponentes da el horizonte de pronóstico. Obsérvese que la información que se recupera tiene mucho sentido debido a que coincide con la accesibilidad a las clases básicas de la matriz, otra forma de ver lo anterior es observar que la matriz que se tiene es reducible, por lo que si se hace una reordenación de los estados se pueden utilizar los resultados sobre la distribución límite.

Nota: Si se utiliza el resultado de la matriz fundamental [16], cuando $p=1 / 2$ para $\pi_{1}$ se tiene que el tiempo medio de absorción es de al menos 6 pasos y para $\pi_{2}$ el tiempo medio de absorción es de al menos 3 pasos. 


\subsection{Sensibilidad al Riesgo: una Extensión del Juego de Apuestas}

\subsubsection{Planteamiento del Problema}

Considérese el siguiente modelo de decisión de Markov.

\section{Modelo}

Para un entero positivo fijo $N$ y un número $p \in[0,1]$ dado, los cincos elementos del modelo se describen a continuación:

(a) $S:=[0,1,2, \ldots, N]$ el espacio de estados del modelo

(b) $A:=\{0,1,2, \ldots,[N / 2]\}$ el espacio de acciones o controles, en donde $[z]$ representa la parte entera de $z$.

(c) $A(s):=$ Para cada $s \in S, A(x)=\{1,2, \ldots, \operatorname{mín}\{s, N-s\}\}$

(d) Se define la siguiente ley de transición $Q=q_{s y}(a)$ para $s \in S$ y $a \in A(s)$ como :

1. $q_{s, s+a}(a)=p$

2. $q_{s, s-a}(a)=q$ donde $q=1-p$

3. $q_{N, 0}(a)=1$

4. $q_{0,0}(a)=1$

(e) La función de respuesta $(\rho)$ en este caso de recompensa por etapa como

$$
R(x . a)=0, x \neq N ; R(N, a)=1 \text {. }
$$


Para este modelo se define la función objetivo $V$ como:

$$
V(\pi, s)=\frac{1}{\lambda} \log \left(E_{s}^{\pi}\left(\sum_{n=0}^{\infty} R\left(S_{n}, A_{n}\right)\right)\right.
$$

Para este caso la $\lambda$ función óptima se define como:

$$
V_{\lambda}(s)=\sup _{\pi \in \Pi}\left\{V_{\lambda}(\pi, s)\right\},
$$

$s \in S$ y así una política se dirá que es $\lambda$ - óptima si se cumple que:

$$
V_{\lambda}\left(\pi^{*}, s\right)=V_{\lambda}(s)
$$

$\forall s \in S$.

Una vez planteado el problema general que se tiene para este caso; el agente va a representar su preferencia al riesgo mediante una función de utilidad $u$, la cual asigna un número real a cada posible resultado. Así que si se tiene que $r_{i}^{a}$ es la recompensa esperada dado que el agente se encuentra en el estado $i$ y selecciona la estrategia $a$, entonces el valor que le asigna la función será igual a $u\left(r_{i}^{a}\right)$. Si $v(n)_{i}$ denota la esperanza máxima del problema en n-etapas entonces la utilidad esperada por el agente será $u\left(v(n)_{i}\right)$. Howard y Mathenson [12] usaron una función de utilidad dada de la siguiente forma:

$u(s)=-(\operatorname{sgn} \bar{\lambda}) \exp (-\bar{\lambda} s)$ donde $\bar{\lambda}$ representa el coeficiente de aversión al riesgo, cabe notar que el tipo de función que se está utilizando es de tipo (CARA) por lo que se está asumiendo que se tiene un coeficiente de aversión al riesgo absoluto constante (7.10). Por lo que, para una una sucesión de recompensas $r_{i_{1}}, r_{i_{2}}, \ldots, r_{i_{n}}$ se tiene que la utilidad viene dada por: $-(\operatorname{sgn} \bar{\lambda}) \exp \left(-\bar{\lambda}\left(r_{i_{1}}+r_{i_{2}}+\ldots+r_{i_{n}}\right)\right)$. Sea $v(n)_{i}$ que denota la utilidad por permanecer n-etapas en el sistema dado que se comenzó en el estado $i$ entonces usando el concepto de "certeza equivalente" Howard 
y Mathenson, [12], probaron que la siguiente recursión es válida para este problema:

$$
v(n)_{i}=\operatorname{máx}_{a \in A} \sum_{j=1}^{N} q_{i j}^{a} \exp \left(-\bar{\lambda} r_{i j}^{a}\right) v(n-1)_{j},
$$

$i=1, \ldots N ; n \in N$. Ahora si se toma:

$\tilde{P}_{i j}^{a}=q_{i j}^{a} \exp \left(\bar{\lambda} r_{i j}^{a}\right)$ se obtiene que la ecuación anterior puede ser reescrita de la siguiente forma:

$$
v(n)_{i}=\operatorname{máx}_{a \in A} \sum_{j=1}^{N} \tilde{P}_{i j}^{a} v(n-1)_{j},
$$

$i=1, \ldots N ; n \in N$ y con los cambios realizados junto con las definición de política, así como en el caso neutral al riesgo es decir $\pi, \mathbb{P}, P(\pi)$ y $v(n)$ como siempre entonces se tiene que:

$$
v(n)=\operatorname{máx}_{\pi \in \mathbb{P}} \tilde{P}(\pi) v(n-1),
$$

$n \in N$. Algo importante a observar en este apartado, es que aún se puede utilizar la técnica de matrices no negativas, pero lo que hay que resaltar es que como ahora se tiene un factor que es afectado por la exponencial esto presenta una dificultad, pero lo que se puede realizar es observar la distribución de la matriz y entonces iterarla no pensando en buscar el eigenvector estrictamente positivo, sino en analizar el comportamiento de la matriz, para el estado $(N, N)$ se introduce un 1, el cual permite recuperar la información de los estados intermedios. Otra cosa que cabe resaltar es que para este caso cuando se alcanza el estado $N$, este da una aportación en la matriz debido a la forma en que se definió la recompensa,

Una vez dado lo anterior, lo que se busca es encontrar una política 
la cual nos de la recompensa máxima, como el espacio de acciones y de controles son iguales que en el ejemplo 5; el número de posibles políticas se calcula de la misma manera que en el caso neutral al riesgo. Un detalle es que para este modelo se tiene que el grado de la matriz $\nu=2$. Aunque lo que cabe resaltar es que se puede seguir aplicando la idea del neutral al riesgo debido a que una vez mas se quiere recuperar la información del estado $N$.

Ejemplo 6. Sea $S=\{0,1,2,3,4\}$ el espacio de estados, sea $A(s)$, $Q$ y $R$ como en la descripción del modelo, se tiene que las matriz de representación de las políticas son:

$$
\pi_{1}:=\left(\begin{array}{ccccc}
1 & 0 & 0 & 0 & 0 \\
q & 0 & p & 0 & 0 \\
0 & q & 0 & p & 0 \\
0 & 0 & q & 0 & p \\
\operatorname{sign}(\bar{\lambda}) * \exp (-\bar{\lambda}) & 0 & 0 & 0 & 1
\end{array}\right) \quad \pi_{2}:=\left(\begin{array}{ccccc}
1 & 0 & 0 & 0 & 0 \\
q & 0 & p & 0 & 0 \\
q & 0 & 0 & 0 & p \\
0 & 0 & q & 0 & p \\
\operatorname{sign}(\bar{\lambda}) * \exp (-\bar{\lambda}) & 0 & 0 & 0 & 1
\end{array}\right)
$$

Para este caso existen varias cosas que mencionar, la primera es que $\bar{\lambda}$ puede ser positiva ó negativa, además de que se tienen los diferentes casos cuando $\mathrm{p}>\mathrm{q}, \mathrm{q}>\mathrm{p}$ y $\mathrm{p}=\mathrm{q}$; así que se hará una separación, indicándose los resultados obtenidos mediante la utilización de la técnica de matrices no negativas.

\begin{tabular}{|c|c|c|c|}
\hline & $\mathrm{p}>\mathrm{q}$ & $\mathrm{p}=\mathrm{q}$ & $\mathrm{p}<\mathrm{q}$ \\
\hline $\bar{\lambda}>0$ & Tímida & Cualquiera & Audaz \\
\hline $\bar{\lambda}<0$ & Tímida & Cualquiera & Audaz \\
\hline
\end{tabular}

En el cuadro anterior, se resumen cuales fueron las estrategias óptimas para cada caso del ejemplo. 
El código "Algoritmo sensible al riesgo" permite solucionar el problema solo dándole el número de estados intermedios +1 , y p (la probabilidad de la transición).

A continuación se presentan los resultados que se obtuvieron para el caso general.

Tómese primero el caso para el cual $\bar{\lambda}>0$ es decir:

$$
\bar{\lambda}=1,2,3,4,5,6,7,8,9,10
$$




\begin{tabular}{|c|c|c|c|c|c|c|c|c|c|}
\hline $\mathrm{P}$ & $Q$ & $\# \bar{N}$ & 3 & 4 & 5 & 6 & 7 & 8 & 9 \\
\hline .1 & .9 & P.O & $(1,2,1)$ & $(1,2,2,1)$ & $\begin{array}{l}(1,1,3,1,1) \\
(1,1,3,2,1) \\
(1,2,3,1,1) \\
(1,2,3,2,1)\end{array}$ & $(1,2,3,3,2,1)$ & $\begin{array}{l}(1,2,1,4,1,2,1) \\
(1,2,1,4,3,2,1) \\
(1,2,3,4,1,2,1) \\
(1,2,3,4,3,2,1)\end{array}$ & $(1,2,3,4,4,3,2,1)$ & $\begin{array}{l}(1,2,2,1,5,1,2,2,1) \\
(1,2,2,1,5,1,3,2,1) \\
(1,2,2,1,5,4,2,2,1) \\
(1,2,2,1,5,4,3,2,1) \\
(1,2,2,4,5,1,2,2,1) \\
(1,2,2,4,5,1,3,2,1) \\
(1,2,2,4,5,4,2,2,1) \\
(1,2,2,4,5,4,3,2,1) \\
(1,2,3,1,5,1,2,2,1) \\
(1,2,3,1,5,1,3,2,1) \\
(1,2,3,1,5,4,2,2,1) \\
(1,2,3,1,5,4,3,2,1) \\
(1,2,3,4,5,1,2,2,1) \\
(1,2,3,4,5,1,3,2,1) \\
(1,2,3,4,5,4,2,2,1) \\
(1,2,3,4,5,4,3,2,1)\end{array}$ \\
\hline .2 & .8 & P.O. & $(1,2,1)$ & $(1,2,2,1)$ & $\begin{array}{l}(1,1,3,1,1) \\
(1,1,3,2,1) \\
(1,2,3,1,1) \\
(1,2,3,2,1)\end{array}$ & $(1,2,3,3,2,1)$ & $\begin{array}{l}(1,2,1,4,1,2,1) \\
(1,2,1,4,3,2,1) \\
(1,2,3,4,1,2,1) \\
(1,2,3,4,3,2,1)\end{array}$ & $(1,2,3,4,4,3,2,1)$ & $\begin{array}{l}(1,2,2,1,5,1,2,2,1) \\
(1,2,2,1,5,1,3,2,1) \\
(1,2,2,1,5,4,2,2,1) \\
(1,2,2,1,5,4,3,2,1) \\
(1,2,2,4,5,1,2,2,1) \\
(1,2,2,4,5,1,3,2,1) \\
(1,2,2,4,5,4,2,2,1) \\
(1,2,2,4,5,4,3,2,1) \\
(1,2,3,1,5,1,2,2,1) \\
(1,2,3,1,5,1,3,2,1) \\
(1,2,3,1,5,4,2,2,1) \\
(1,2,3,1,5,4,3,2,1) \\
(1,2,3,4,5,1,2,2,1) \\
(1,2,3,4,5,1,3,2,1) \\
(1,2,3,4,5,4,2,2,1) \\
(1,2,3,4,5,4,3,2,1)\end{array}$ \\
\hline .3 & .7 & P.O & $(1,2,1)$ & $(1,2,2,1)$ & $\begin{array}{l}(1,1,3,1,1) \\
(1,1,3,2,1) \\
(1,2,3,1,1) \\
(1,2,3,2,1)\end{array}$ & $(1,2,3,3,2,1)$ & $\begin{array}{l}(1,2,1,4,1,2,1) \\
(1,2,1,4,3,2,1) \\
(1,2,3,4,1,2,1) \\
(1,2,3,4,3,2,1)\end{array}$ & $(1,2,3,4,4,3,2,1)$ & $\begin{array}{l}(1,2,2,1,5,4,2,2,1) \\
(1,2,2,1,5,4,3,2,1) \\
(1,2,2,4,5,4,2,2,1) \\
(1,2,2,4,5,4,3,2,1) \\
(1,2,3,1,5,4,2,2,1) \\
(1,2,3,1,5,4,3,2,1) \\
(1,2,3,4,5,4,2,2,1) \\
(1,2,3,4,5,4,3,2,1)\end{array}$ \\
\hline
\end{tabular}




\begin{tabular}{|c|c|c|c|c|c|c|c|c|c|}
\hline .4 & .6 & P.O & $(1,2,1)$ & $(1,2,2,1)$ & $\begin{array}{l}(1,1,3,1,1) \\
(1,1,3,2,1) \\
(1,2,3,1,1) \\
(1,2,3,2,1)\end{array}$ & $(1,2,3,3,2,1)$ & $\begin{array}{c}(1,2,1,4,1,2,1) \\
(1,2,1,4,3,2,1) \\
(1,2,3,4,1,2,1) \\
(1,2,3,4,3,2,1)\end{array}$ & $(1,2,3,4,4,3,2,1)$ & $\begin{array}{l}(1,2,2,1,5,1,2,2,1) \\
(1,2,2,1,5,1,3,2,1) \\
(1,2,2,1,5,4,2,2,1) \\
(1,2,2,1,5,4,3,2,1) \\
(1,2,2,4,5,1,2,2,1) \\
(1,2,2,4,5,1,3,2,1) \\
(1,2,2,4,5,4,2,2,1) \\
(1,2,2,4,5,4,3,2,1) \\
(1,2,3,1,5,1,2,2,1) \\
(1,2,3,1,5,1,3,2,1) \\
(1,2,3,1,5,4,2,2,1) \\
(1,2,3,1,5,4,3,2,1) \\
(1,2,3,4,5,1,2,2,1) \\
(1,2,3,4,5,1,3,2,1) \\
(1,2,3,4,5,4,2,2,1) \\
(1,2,3,4,5,4,3,2,1)\end{array}$ \\
\hline .5 & .5 & P.O & $\pi_{i}$ & $\pi_{i}$ & $\pi_{i}$ & $\pi_{i}$ & $\pi_{i}$ & $\pi_{i}$ & $\pi_{i}$ \\
\hline .6 & .4 & $\mathrm{P} . \mathrm{O}$ & $\pi_{1}$ & $\pi_{1}$ & $\pi_{1}$ & $\pi_{1}$ & $\pi_{1}$ & $\pi_{1}$ & $\pi_{1}$ \\
\hline .7 & .3 & P.O & $\pi_{1}$ & $\pi_{1}$ & $\pi_{1}$ & $\pi_{1}$ & $\pi_{1}$ & $\pi_{1}$ & $\pi_{1}$ \\
\hline .8 & .2 & P.O & $\pi_{1}$ & $\pi_{1}$ & $\pi_{1}$ & $\pi_{1}$ & $\pi_{1}$ & $\pi_{1}$ & $\pi_{1}$ \\
\hline .9 & .1 & P.O & $\pi_{1}$ & $\pi_{1}$ & $\pi_{1}$ & $\pi_{1}$ & $\pi_{1}$ & $\pi_{1}$ & $\pi_{1}$ \\
\hline
\end{tabular}

En donde $\pi_{1}$ denota la política en la que siempre se avanza de uno en uno. $(1, \ldots, 1)$, además cuando $p>q$ la política óptima es la anterior que se conoce en el ambiente probabilístico como la tímida; cabe notar que cuando $q>p$ hay una política la cual aparece en todos los casos y esta es la conocida como la audaz; esta política elige una acción más agresiva, pues cuando el sistema está en un estado $s \leq N / 2$, se elige la acción que le permitiría avanzar el doble del estado actual y cuando sistema está en $s \geq N / 2$, su elección es todo lo necesario para que, de avanzar se llegue al objetivo $N$. Nota: Para el caso en el cual $p=1 / 2$, se tiene que la política $\pi_{i}$ denota que para cualquier $i$, la selección que se elija es óptima.

Así que se pueden resumir los resultados como: 
Si $\mathrm{p}>\mathrm{q}$ entonces la política óptima es la tímida y es única. Si $q>$ p entonces la política óptima es la audaz y para algunos casos especiales se tiene que esta no es única. $\mathrm{Si} \mathrm{q}=\mathrm{p}$ entonces cualquier política es óptima.

Ahora para el caso en que $\bar{\lambda}<0$ y si se realiza el mismo procedimiento es decir $\bar{\lambda}=-1,-2,-3,-4,-5,-6,-7,-8,-9,10$. Se obtienen los mismos resultados que en la tabla anterior, que no causa una gran sorpresa debido a que intrínsecamente lo que está de fondo es el juego neutral al riesgo.

Así que se pueden enumerar los resultados como: Para $\bar{\lambda}<0$ Si $\mathrm{p}>\mathrm{q}$ entonces la política óptima es la tímida y es única. Si $q>$ p entonces la política óptima es la audaz y para algunos casos especiales se tiene que esta no es única. $\mathrm{Si} \mathrm{q}=\mathrm{p}$ entonces cualquier política es óptima. 


\subsubsection{Algoritmos}

\section{Algoritmo neutral al riesgo}

$$
\begin{aligned}
& 1 \text { clear } \\
& 2 \text { clc } \\
& 3 \text { tic } \\
& 4 \mathrm{~N}=5 \\
& 5 \mathrm{p}=.8 \\
& 6 \mathrm{q}=1-\mathrm{p} ; \\
& 7 \mathrm{w}=[\mathbf{z e r o s}(1, \mathrm{~N})] \\
& 8 \text { for } \quad \mathrm{i}=1: \mathrm{N}-1 \\
& 9 \quad \quad \mathrm{~b}=[\mathrm{i} \quad \mathrm{N}-\mathrm{i}] \\
& 10 \quad \quad \mathrm{a}(\mathrm{i})=\min (\mathrm{b}) ;
\end{aligned}
$$

\section{1 end}

13 for $\mathrm{j}=1$ :length (a)

$14 \quad x(j)=\{1: a(j)\}$;

15 end

16

$17 \mathrm{u}=\operatorname{allcomb}(\mathrm{x}\{:\})$;

$18[\mathrm{u} 1, \mathrm{u} 2]=\mathbf{s i z e}(\mathrm{u})$;

19

$20 \mathrm{n}=\operatorname{length}(\mathrm{a})+3$;

21

22 for $r=1: u 1$

$23 \mathrm{~A}=\operatorname{zeros}(\mathrm{n})$;

$24 \mathrm{~A}(1,1)=1$;

$25 \mathrm{~A}(\mathrm{n}, \mathrm{n})=1$; 
$26 \mathrm{~A}(\mathrm{n}-1, \mathrm{n})=1$;

$27 \mathrm{~A}(\mathrm{n}-1,1)=1$;

28 for $i=2: n-2$

29

for $\mathrm{j}=1: \mathrm{n}$

30

if $\mathrm{i}-\mathrm{j}=\mathrm{u}(\mathrm{r}, \mathrm{i}-1)$;

31

$A(i, j)=q \quad ;$

32

elseif $\mathrm{i}-\mathrm{j}=-\mathrm{u}(\mathrm{r}, \mathrm{i}-1)$;

33

$\mathrm{A}(\mathrm{i}, \mathrm{j})=\mathrm{p}$;

34

end

35

end

$36 \quad$ end

$37 \quad \%=\operatorname{sparse}(A)$;

$38 \quad \mathrm{~B}(\mathrm{r})=\{\mathrm{A}\}$;

39 end

40

$41 \mathrm{~m}=(\mathrm{N} *(\mathrm{~N}+11))$

42

43 for $\mathrm{i}=1: \mathrm{u} 1$

$44 \quad \mathrm{~B} 1(\mathrm{i})=\left\{\mathrm{B}\{\mathrm{i}\}^{\wedge} \mathrm{m}\right\}$;

45 end

46

$47 \quad \mathrm{~B} 3=\operatorname{zeros}(\mathrm{N}-2, \mathrm{r})$;

48 for $\mathrm{j}=1: \mathrm{N}-1$

49 for $\mathrm{i}=1: \mathrm{u} 1$

$50 \quad \mathrm{~B} 2(\mathrm{i})=\max (\mathrm{B} 1\{\mathrm{i}\}(\mathrm{j}+1, \mathrm{~N}+2))$;

51 end

$52 \quad \mathrm{~B} 3(\mathrm{j},:)=\mathrm{B} 2$;

53 end 
54

$55 \mathrm{t} 1=[]$

$56 \max \max (\mathrm{B} 3,[], 2)$;

$57 \mathrm{j}=0$;

58 for $\mathrm{i}=1: \mathrm{u} 1$

59 if $\max -\mathrm{B} 3(:, \mathrm{i})<10^{\wedge}-14$

$60 \quad \mathrm{j}=\mathrm{j}+1$;

$61 \quad \mathrm{t} 1(\mathrm{j})=(\mathrm{i})$;

62 end

63 end

$64 \mathrm{u}(\mathrm{t} 1,:)$

65 toc 


\title{
Algoritmo sensible al riesgo
}

\author{
1 clear \\ 2 clc \\ 3 tic \\ 4 gamma -5 \\ $5 \mathrm{~N}=7$ \\ $6 \mathrm{~W}=[\operatorname{zeros}(1, \mathrm{~N})]$; \\ 7 for $\mathrm{i}=1: \mathrm{N}-1$ \\ $8 \quad \mathrm{~b}=\left[\begin{array}{ll}\mathrm{i} & \mathrm{N}-\mathrm{i}\end{array}\right]$; \\ $9 \quad \mathrm{a}(\mathrm{i})=\min (\mathrm{b})$; \\ 10 end
}

11

12 for $\mathrm{j}=1$ : length (a)

$13 \quad \mathrm{x}(\mathrm{j})=\{1: \mathrm{a}(\mathrm{j})\}$;

14 end

15

$16 \mathrm{u}=\operatorname{allcomb}(\mathrm{x}\{:\})$;

$17[\mathrm{u} 1, \mathrm{u} 2]=\mathbf{s i z e}(\mathrm{u})$;

18

19

$20 \mathrm{p}=.6$;

$21 \mathrm{q}=1-\mathrm{p}$;

$22 \mathrm{n}=$ length $(\mathrm{a})+2$;

23

24 for $r=1: u 1$

$25 \mathrm{~A}=\operatorname{zeros}(\mathrm{n}-1)$;

$26 \mathrm{~A}(1,1)=1$;

$27 \mathrm{~A}(\mathrm{n}, \mathrm{n})=1$; 
$28 \%(n-1, n-1)=0$;

$29 \mathrm{~A}(\mathrm{n}, 1)=\mathbf{s i g n}(\operatorname{gamma}) * \exp (-\operatorname{gamma})$;

30 for $\mathrm{i}=2: \mathrm{n}-1$

31

32

$$
\text { for } \mathrm{j}=1: \mathrm{n}
$$

2

$$
\begin{gathered}
\text { if } \quad \mathrm{i}-\mathrm{j}=\mathrm{u}(\mathrm{r}, \mathrm{i}-1) ; \\
\mathrm{A}(\mathrm{i}, \mathrm{j})=\mathrm{q} ;
\end{gathered}
$$
elseif $\mathrm{i}-\mathrm{j}=-\mathrm{u}(\mathrm{r}, \mathrm{i}-1)$; $A(i, j)=p$; end 
$56 \mathrm{t} 1=[]$;

$57 \max -\max (\mathrm{B} 3,[], 2)$;

$58 \mathrm{j}=0$;

59 for $i=1: u 1$

60 if $\max -\mathrm{B} 3(:, \mathrm{i})<10^{\wedge}-6$

$61 \quad \mathrm{j}=\mathrm{j}+1$;

$62 \quad \mathrm{t} 1(\mathrm{j})=(\mathrm{i})$;

63 end

64 end

$65 \mathrm{u}(\mathrm{t} 1,:)$

66 toc 


\section{Capítulo 6}

\section{Conclusiones y Perspectivas}

El trabajo de esta tesis se centró en las matrices finitas no negativas y en aplicaciones, tanto en el campo de la economía, con las matrices de Leontief, como en el de control estocástico, específicamente en los procesos de control de Markov con recompensa total esperada. Como se pudo observar la teoría expuesta permite, dependiendo del problema que se aborde, enlazar distintas áreas de las matemáticas como el álgebra lineal, los procesos estocásticos, los problemas de control estocásticos, el análisis funcional, el cálculo y algunos resultados topológicos, por lo que es de vital importancias resaltar que lo mejor que se puede hacer es buscar la interacción entre estas áreas.

\subsection{Conclusiones}

1. En la aplicación sobre las matrices de Leontief, como se pudo observar la aproximación y la resolución que se le puede dar, en muchas ocasiones, depende del enfoque o la información que se quiera ob- 
tener; es importante notar que la determinación de las matrices de insumo-producto puede ser un arduo trabajo, por lo que el supuesto de tenerlas dadas permite la aplicación de la técnica de matrices no negativas de forma inmediata. Nótese que aunque aquí se trabajó para un caso de dos empresas y el banco, también se puede desarrollar un ejemplo para el caso de $\mathrm{N}$ empresas con $\mathrm{N}>2$, siempre y cuando se respete la irreducibilidad de la matriz porque en caso contrario se deberá abordar el tema con matrices reducibles.

2. En el caso de la aplicación al control estocástico, es de suma importancia recalcar que los resultados que se obtuvieron son para un ejemplo del caso total esperado; es relevante notar que el número de estados para los que se presentan los resultados es 11, aunque se logró hacer lo mismo para cuando había 14 estados; sin embargo una problemática que surgió es que cuando el número de estados crece por ejemplo 15, el número de posibles estrategias es: 203212800. Una vez hecho lo anterior se necesita calcular la matriz de transición y después la comparación sobre las políticas para obtener la óptima. Recuérdese que en el camino todavía se necesita una iteración de estas matrices a la cota de pronóstico, por lo que para cuando el número de estados crece el problema aparece más en el caso de falta de memoria en las computadoras que en la debilidad de la técnica. Cabe señalar que está técnica fue abandonada por los años 70 y ahora con el desarrollo de la tecnología está volviendo a aparecer en distintos ámbitos.

3. Una ventaja que ofrece esta metodología de matrices no negativas, descrita en este trabajo, es que no sólo permitió decir cuál era la estrategia óptima para el jugador sino que en algunos casos mostró la 
existencia de otras soluciones, con lo que permitió decir que existían casos para los cuales la solución no es única. Esto es algo que no se conocía en la literatura para ambos casos, neutral y sensible al riesgo; así mismo se puede dar información sobre los valores a los cuales converge.

\subsection{Perspectivas}

1. Un punto interesante es que para este trabajo solo se utilizó la técnica para los casos cuando $\sigma=\nu=1$ (recuérdese que $\sigma$ representa el radio espectral y $\nu$ representa el grado) en el caso de la recursión dinámica, sin embargo esta herramienta sobre matrices no negativas ya está generalizada para cuando $\nu \neq 1$ la cual se puede encontrar en [31]. Por lo que sería relevante buscar ejemplos en donde ésta pueda aplicarse en dimensión diferente a 1.

2. Un hecho que es esencial es que la herramienta que se utilizó está hecha para matrices no negativas en general, aunque en esta tesis se centró en problemas que involucran matrices de transición estocástica. Por tanto sería interesante abordar problemas que no sean de corte estocástico.

3. Cabe señalar que la técnica de matrices no negativas permite abordar muchos problemas y que sus aplicaciones pueden ser llevadas a un lugar más amplio como lo pueden ser los espacios de estados infinito numerables [31]. 


\section{Capítulo 7}

\section{Apéndices}

Este capítulo permite complementar los detalles que se eleboraron en la tesis. La subsección sobre un Resultado auxiliar permite demostrar la existencia de la solución al sistema de ecuaciones planteadas. La subsección sobre el radio espectral y el teorema de Perron Frobenius esta compuesta por las demostraciones así como la formulación de estos resultados ampliamente conocidos en el ambiente del Álgebra Lineal. La subsección sobre Sensibilidad al Riesgo es un paseo sobre su definición y su fundamentación desde las ideas básicas, hasta su medición para terminar en su representación mediante la función de utilidad.

\subsection{Un Resultado Auxiliar}

Lema 7.1.1. Sea $P$ una matriz cuadrada que tiene radio espectral $\sigma$ y eigenvector derecho estrictamente positivo asociado con $\sigma$. Más aún $(r(1), \ldots r(k-1))$ es una sucesión de vectores dada. Entonces existe una solución $(y(1), \ldots, y(k))$ del conjunto de ecuaciones 


$$
\left\{\begin{array}{cc}
P y(k) & =\sigma y(k) \\
P y(k-1)+r(k-1) & =\sigma y(k-1)+y(k) \\
\vdots+\quad \vdots & \vdots+\quad \vdots \\
P y(1)+r(1) & =\sigma y(1)+y(2)
\end{array}\right.
$$

Demostración. Por el lema $2.2 .9, P^{*}$ existe y además $\left(\sigma I-P+P^{*}\right)$. La iteración en la primera ecuación nos da:

$$
\sigma^{-n} P^{n} y(k)=y(k)
$$

$n \in \mathbb{N}$ entonces por la definición de $P^{*}$ se tiene que:

$$
P^{*} y(k)=y(k)
$$

Ahora multiplicando los dos lados de 7.1 por $P^{*}$ se obtiene que:

$$
P^{*} r(l-1)=P^{*} y(l)
$$

$l=2, \ldots, k$. Como se puede notar falta definir que sucede con respecto a y(1) así que considérese:

$$
P^{*} y(1)=\underline{0}
$$

Con lo anterior, si se combinan todas las ecuaciones 7.1, 7.2, 7.3 y 7.4 y se recuerda que la matriz $\left(\sigma I-P+P^{*}\right)$ es no singular entonces existe una solución $(y(1), y(2), \ldots, y(k))$ de 7.1-7.4 como sigue a continuación:

$$
\begin{aligned}
& y(k) \quad=P^{*} r(k-1) \\
& y(k-1)=\left(\sigma I-P+P^{*}\right)^{-1}\left(r(k-1)+P^{*} r(k-2)-y(k)\right) \\
& y(2) \quad=\left(\sigma I-P+P^{*}\right)^{-1}\left(r(2)+P^{*} r(1)-y(3)\right) \\
& y(1) \quad=\left(\sigma I-P+P^{*}\right)^{-1}(r(1)-y(2))
\end{aligned}
$$

Obsérvese que la solución a 7.1 no necesariamente es única. $\square$. 


\subsection{Radio Espectral}

Durante esta sección $\mathbb{X}$ denota un espacio $\mathbf{B}$ complejo y $T$ un operador lineal acotado. Se excluye el caso cuando $\mathbb{X}=0$

Definición 7.2.1. Se le conoce como el resolvente de un conjunto $\rho(T)$ de $T$ al conjunto de los números complejos $\lambda$ para el cual $(\lambda I-T)^{-1}$ existe y además es un operador acotado con dominio $\mathbb{X}$. El espectro $\sigma(T)$ de $T$ es el complemento de $\rho(T)$.

La función $R(\lambda ; T)=(\lambda I-T)^{-1}$ definida en $\rho(T)$ es conocida como la función resolvente, o simplemente como la resolvente de $T$.

Lema 7.2.2. El conjunto resolvente $\rho(T)$ es abierto y la función $R(\lambda ; T)$ es analítica en $\rho(T)$

\section{Demostración:}

Sea $\lambda$ un punto fijo de $\rho(T)$, y sea $\mu$ cualquier número complejo para el cual $|\mu|<|R(\lambda ; T)|^{-1}$. Probaremos que con esto se tiene que $\lambda+\mu \in \rho(T)$. Una consideración heurística nos propone que basados en una analogía con las series geométricas que si $(\lambda+\mu) I-T=\mu I+(\lambda I-T)$ tiene inversa entonces está viene dada por las series:

$S(\mu)=\sum_{k=0}^{\infty}(-\mu)^{k}(\lambda I-T)^{-(k+1)}=\sum_{k=0}^{\infty}(-\mu)^{k} R(\lambda ; T)^{k+1}$

Como $|\mu R(\lambda ; T)|<1$ entonces esta serie converge. Además como $S(\mu)$ conmuta con $T$ y se tiene que:

$$
\begin{aligned}
((\lambda+\mu) I-T) S(\mu) & =(I-T) S(\mu)+\mu S(\mu) \\
& =\sum_{k=0}^{\infty}\left\{(-\mu R(\lambda ; T))^{k}-(-\mu R(\lambda ; T))^{k+1}\right\} \\
& =I
\end{aligned}
$$


de donde se sigue que $\lambda+\mu \in \rho(T)$ y se tiene que $R(\lambda+\mu ; T)=S(\mu)$ es analítica en el punto $\mu=0$.

Corolario 7.2.3. Si $d(\lambda)$ es la distancia de $\lambda$ al radio espectral $\sigma(T)$ entonces

$$
|R(\lambda ; T)| \geq 1 / d(\lambda)
$$

$\lambda \in \rho(T)$. Y así $|R(\lambda ; T)| \rightarrow \infty$ cuando $d(\lambda) \rightarrow 0$ y además el conjunto resolvente es el dominio natural de analiticidad de $R(\lambda ; T)$.

\section{Demostración:}

Por el lema 7.2.2 se tiene que si $|\mu|<|R(\lambda ; T)|^{-1}$ entonces $\lambda+\mu \in \rho(T)$. De donde $d(\lambda) \geq|R(\lambda ; T)|^{-1}$, con lo que se obtiene el resultado.

Lema 7.2.4. El conjunto cerrado $\sigma(T)$ es acotado y no vacío. Más aún el $\sup |\sigma(T)|=\lim _{n \rightarrow \infty} \sqrt[n]{\left|T^{n}\right|} \leq|T|$. Para $|\lambda|>\sup |\sigma(T)|$ la serie $R(\lambda ; T)=$ $\sum_{n=0}^{\infty} T^{n} / \lambda^{n+1}$ converge al operador uniforme en el sentido topológico.

\section{Demostración:}

Sea $f(\lambda)=\sum_{n=0}^{\infty} T^{n} / \lambda^{n+1}$, por el Teorema de Liuville se tiene que el dominio de convergencia es $\mathbf{D}=\left\{\lambda|| \lambda \mid>\limsup _{n \rightarrow \infty} \sqrt[n]{\left|T^{n}\right|}\right\}$. Como en la prueba del lema 7.2.2 se revisará que $f(\lambda)(\lambda I-T)=(\lambda I-T) f(\lambda)=I$ para $\lambda \in D$, de donde $D \subseteq \rho(T)$ y de esto tenemos que $\sigma(T)$ es acotado. Como $\sigma(T)$ es el dominio natural de analiticidad para $R(\lambda: T)$, utilizando el corolario 7.2.3 se tiene que la serie de Laurent para $R(\lambda ; T)$ tiene dominio de convergencia para $|\lambda|>\sup |\sigma(T)|$. Entonces $\sup |\sigma(T)|=\lim \sup \sqrt[n]{\left|T^{n}\right|}$. El siguiente paso de la demostración será demostrar que sup $|\sigma(T)| \leq \operatorname{lím}$ inf $\sqrt[n]{\left|T^{n}\right|}$. Nótese que si $\lambda$ es un punto arbitrario de $\sigma(T)$, entonces $\lambda^{n} \in \sigma\left(T^{n}\right)$; utilizando factorización se tiene que:

$\left(\lambda^{n} I-T^{n}\right)=(\lambda I-T) P_{n}(T)=P_{n}(T)(\lambda I-T)$

muestra que si $\left(\lambda^{n} I-T^{n}\right)$ tiene inverso acotado y entonces $(\lambda I-T)$ también 
lo tendrá. Se tiene que: $|\lambda|^{n} \leq\left|T^{n}\right|$ y de aquí que $\sup |\sigma(T)| \leq\left|T^{n}\right|^{1 / n}$. Aún falta por demostrar que el espectro es no vacío. Supóngase que $\sigma(T)=\emptyset$ esto implica que $R(\lambda ; T)$ es entera y entonces como se había visto por la expansión de Laurent que es analítica en el infinito entonces se sigue por el teorema de Liuville que $R(\lambda ; T)$ es constante, por lo que el coeficiente de $\lambda^{-1}$ de la expansión de Laurent desaparece y entonces se tiene que $\mathrm{I}=0$ la cual contradice la hipótesis de que $\mathbb{X} \neq 0$.

Definición 7.2.5. La cantidad

$r(T)=\sup |\sigma(T)|=\lim _{n \rightarrow \infty} \sqrt[n]{\left|T^{n}\right|}$ es llamado el radio espectral de $T$

\subsection{Teorema de Perron Frobenius}

Definición 7.3.1. Se define el radio espectral de una matriz cuadrada $P$ $\sigma(P)=\operatorname{máx}\{|\lambda| \mid \lambda$ es un eigenvalor de $P\}$

Definición 7.3.2. Sea A una matriz cuadrada no negativa e irreducible. Sea d el número de valores propios distintos de $A$ de valor absoluto igual al radio espectral $\sigma(A)$. Se dice que $A$ es una matriz primitiva si $d=1$ ó que no es primitiva si $d>1$.

Proposición 7.3.3. Si A es una matriz cuadrada con entradas en los reales de orden $n$, no negativa e irreducible entonces:

$$
(I+A)^{n-1}>0
$$

Demostración:

Considérese un vector $y \in R^{n}, y \geq 0$ y escríbase

$$
z=(I+A) y=y+A y
$$


Como $A \geq 0$, el producto $A y \geq 0$, por lo que $z$ tiene al menos tantos elementos no nulos como $y$. Ahora, si $y$ aún no es positivo, el vector $z$ tiene al menos un elemento no nulo más que $y$. Si $P$ es una matriz de permutación tal que:

$$
P y=\left(\begin{array}{l}
u \\
0
\end{array}\right) .
$$

y $\mathrm{u}>0$ entonces:

$$
P z=\left(\begin{array}{l}
u \\
0
\end{array}\right)+P A P^{t}\left(\begin{array}{l}
u \\
0
\end{array}\right),
$$

porque $P P^{t}=I$. Si se realiza una partición de $P z$ y $P A P^{t}$ de forma consistente a la de $y$, es decir:

$$
P z=\left(\begin{array}{c}
v \\
w
\end{array}\right), P A P^{t}\left(\begin{array}{ll}
A_{11} & A_{12} \\
A_{21} & A_{22}
\end{array}\right),
$$

visto lo anterior entonces se tiene que:

$$
\left(\begin{array}{c}
v \\
w
\end{array}\right)=\left(\begin{array}{l}
u \\
0
\end{array}\right)+\left(\begin{array}{ll}
A_{11} & A_{12} \\
A_{21} & A_{22}
\end{array}\right)\left(\begin{array}{l}
u \\
0
\end{array}\right),
$$

de donde:

$$
v=u+A_{11} u, w=A_{21} u .
$$


Como la matriz $P A P^{t}$ es no negativa e irreducible, entonces en particular se tiene que $A_{11} \geq 0, A_{21} \geq 0$ y además $A_{21} \neq 0$ por lo que $v>0$, y $w \geq 0$. Como $\mathrm{u}>0$ se obtiene que $w \neq 0$. Así, $z$ tiene al menos una componente más no nula que $y$.

Si $z=(I+A) y$ no es aún un vector positivo, entonces si se repite el argumento con $z$, se tendría que $(I+A)^{2} y$ tiene al menos dos componentes positivas más que $y$. Después de a lo más n-1 pasos se encuentra que:

$$
(I+A)^{n-1} y>0 .
$$

para cualquier vector $y \geq 0, y \neq 0$. Tómese $y=e_{j}$ (en donde $e_{j}$ denota el vector que tiene todas sus entradas cero salvo la j ésima, en la cual tiene un 1 ), para $j=1,2, \ldots, n$ se obtiene el resultado.

Proposición 7.3.4. Si $A \geq 0$ es no negativa irreducible. Entonces existe $k \in \mathbb{N}$ tal que $A^{k}>0$.

\section{Demostración:}

Como $A$ es no negativa e irreducible por la proposición anterior se tiene que: $(I+A)^{n-1} y>0$, si se considera $A(I+A)^{n-1} y$ esto es positivo debido a que sería el producto de un vector por una matriz positiva, lo que da por resultado algo positivo así que si se propone $k=n$ la cual depende de la proposición anterior, se obtiene el resultado.๑.

Considérese A una matriz no negativa e irreducible y defínase la siguiente función:

$r: \Re^{n} \rightarrow \Re$ dada sobre los vectores $x \geq 0$ por:

$$
r(x)=\operatorname{linf}_{\substack{1 \leq i \leq n \\ x_{i} \neq 0}} \frac{(A x)_{i}}{x_{i}},
$$

donde $(A x)_{i}$ representa la i-ésima componente del vector $A x$. Entonces $r(x) \geq 0$, y para $j=1, \ldots, n$ se tiene que $r(x) x_{j} \leq(A x)_{j}$. De donde 
$r(x) x \leq A x$ y además $r(x)$ es el mayor número $\rho$ tal que $\rho x \leq A x$.

Lema 7.3.5. Sea $A=\left(a_{i j}\right)$ no negativa e irreducible y $x=(1,1, \ldots, 1)^{t}$ entonces

$$
r(x)=\min _{i \leq i \leq n} \sum_{i=1}^{n} a_{i k}
$$

\section{Demostración:}

El resultado se sigue de tomar el vector " $x=(1,1, \ldots, 1)^{t}$ ", aplicándolo directamente a la ecuación (7.5).

Teorema 7.3.6. (Perron-Frobenius) Sea A una matriz cuadrada irreducible y no negativa, entonces:

1. A tiene, al menos, un eigenvalor s real y positivo, con un eingenvector z asociado a s positivo;

2. s tiene multiplicidad algebraica igual a 1 ;

3. Si $\lambda_{i}$ es un eigenvalor de $A$, entonces $\left|\lambda_{i}\right| \leq s ; y$

4. Si A tiene una fila de elementos no nulos entonces $\left|\lambda_{i}\right|<s$.

\section{Demostración:}

Sea $L$ el dominio de la función $r$ (7.5), esto es, el conjunto de los vectores no nulos y no negativos de orden $n$, defínase el número $s$ como:

$$
s=\sup _{x \in L} r(x)
$$

Nótese que el número $s$ no varía si en lugar de $x$ se toma un múltiplo $\alpha x$ para $\alpha>0$. Sea $M$ el conjunto de vectores $x$ tales que $x \geq 0$ y $\|x\|_{2}=1$, obsérvese que $M \subset L \mathrm{y}$ 


$$
s=\sup _{x \in M} r(x)
$$

Si se tuviera que la función $r$ fuera continua en $M$ entonces se alcanzaría el supremo, pero ésta puede tener discontinuidades. Así que si se considera el conjunto $N=\left\{(I+A)^{n-1} x \mid x \in M\right\}$. Por la proposición 7.3.3 todo elemento de $N$ es un vector positivo, por lo que $N \subset L$. Además, $N$ es una imagen continua de $M$ bajo la función $(I+A)^{n-1} x$, por lo que es cerrado y acotado. Además la función $r(\cdot)$ es continua en $N$, debido a que no hay denominadores nulos. De donde para cualquier $x \in M \mathrm{y}$ su correspondiente vector $y$ se tiene que:

$$
r(x) y=r(x)(I+A)^{n-1} x \leq(I+A)^{n-1} A x .
$$

porque $r(x) x \leq A x$. Por tanto $r(x) y \leq A y$ para todo $y \in N$. Recuérdese que $r(y)$ es el mayor número $\rho$ tal que $\rho y \leq A y$. De donde se obtiene que $r(x) \leq y \mathrm{y}$

$$
s=\sup _{x \in M} r(x) \leq \operatorname{máx}_{y \in N} r(y)
$$

Pero $N \subset L$, por lo que:

$$
\operatorname{máx}_{y \in N} r(y) \leq \sup _{x \in L} r(x)=s .
$$

En conclusión,

$$
s=\operatorname{máx}_{y \in N} r(y)
$$

de donde existe $y>0$ tal que $s=r(y)$, el cual no necesariamente es único. De hecho puede haber otros vectores en el conjunto $L$ donde la función $r$ alcance el valor $s$. En caso de existir, a estos valores se les denominará los vectores extremales de $A$. Así, un vector no nulo $z \geq 0$ es extremal de $A$ si $r(z)=s$ o lo que es equivalente, $s z \leq A z$. 
Si $v=(1, \ldots, 1)$ entonces $r(v)=\min _{1 \leq i \leq n} \sum_{k=1}^{n} a_{i k}>0$, porque no puede tener una fila de ceros. Como $s \geq r(v)$ se tiene que $s>0$.

Sea $z$ un vector extremal y sea $w=(I+A)^{n-1} z$. Sin pérdida de generalidad se puede suponer que $z \in M$, entonces por la proposición 7.3.3 se tiene que $w>0$, y además $w \in N$. De donde se sigue entonces que $A x-s z \geq 0$. Si $A z-s z \neq 0$ entonces:

$$
(I+A)^{n-1}(A z-s z)>0 .
$$

pero entonces $A w-s w>0$, o bien $s w<A w$, lo que implica que $s<r(w)$, lo anterior es una contradicción. Por lo tanto, para un vector extremal $z$ se tiene que $A z=s z$, lo que significa que $z$ es un eigenvector asociado a s. Además

$$
w=(I+A)^{n-1} z=(1+s)^{n-1} z .
$$

como $w>0$ y $r>0$ se tiene que $z>0$.

Entonces lo que falta por probar es que los otros eigenvalores de A tienen un módulo menor o igual que $s$. Si $y$ es un vector, se denota por $|y|$ al vector formado por los módulos de sus componentes. Análogamente se hace para las matrices. Sea $\alpha$ un eigenvalor de $A$ y y el eigenvector asociado. Entonces se tiene que:

$$
|\alpha||y|=|\alpha y|=|A y| \leq|A||y| .
$$

De donde $|\alpha| \leq r(|y|) \leq s$. Lo siguiente a revisar es la multiplicidad algebraica de $s$. Sea $z$ un eigenvector asociado a $s$.

Tenemos que $A z=s z$ y $z$ no es nulo, por lo que:

$$
s|z| \leq A|z| .
$$


Lo que implica que $|z|$ es un vector extremal (eigenvector positivo de $s$ ), de donde $z>0$. Analizando a $z$ se tiene que todas sus componentes $z_{i}$ no son nulas. Supóngase que existen dos eigenvectores asociados a $s$ independientes $z_{1}$ y $z_{2}$.

Entonces se podrían encontrar escalares $\alpha, \beta$ tales que el eigenvector $\alpha z_{1}+\beta z_{2}$ tenga alguna componente nula. Pero esto contradice que el eigenvector tiene todas sus entradas no nulas. Por lo tanto, el espacio de eigenvectores asociado a $s$ es de dimensión 1. Así que se puede ver como: $V_{1}(s)=\langle z\rangle$.

Lo siguiente a observar es que no existen eigenvectores generalizados de orden 2 (es decir, elementos del $\operatorname{ker}\left(\left(s I-A^{2}\right)\right)$ asociados a $s$. Sean $x_{1}>0$ y $y>0$ eigenvectores positivos de $A$ y $A^{t}$, respectivamente asociados a $s$. Entonces:

$$
(s I-A) x_{1}=0, \quad\left(s I-A^{t}\right) y=0 .
$$

Supóngase que existe un eigenvector generalizado $x_{2}$ tal que $(s I-A) x_{2}=$ $x_{1}$. Como $y^{t}(s I-A)=0$, se tiene que $y^{t} x_{1}=0$, lo cual contradice que $x_{1}$ y $x_{2}$ son positivos. De donde, se obtiene que la multiplicidad algebraica de $s$ es 1 . Supóngase ahora que $A$ tiene una fila de elementos no nulos, por ejemplo, la k-ésima. Ahora sea $\lambda$ un eigenvalor de $A$ tal que $|\lambda|=s$ y $v$ su eigenvector asociado, entonces se tiene que:

$$
s|v|=|\lambda v|=|A v| \leq A|v|
$$

Por lo tanto, $|v|$ es un vector extremal, y así se ha visto que en tal caso no sólo se tiene la desigualdad sino también se tiene la igualdad. De donde se obtiene que:

$$
s|v|=A|v|
$$

Entonces $|v| \in\left\langle z_{0}\right\rangle$. Por lo que se concluye que: 


$$
|A v|=|\lambda v|=s|v|=A|v|
$$

y como la intención es observar la fila k, restringiéndose sólo a ésta se tiene que:

$$
\left|\sum_{j=1}^{n} a_{k j} v_{j}\right|=\sum_{j=1}^{n}\left|v_{j}\right|,
$$

y si $a_{k j}>0$, con $j=1, \ldots, n$ lo cual sólo es posible cuando $v$ es un múltiplo de un vector no negativo (i.e. cuando todas las componentes $v_{i}$ son simultáneamente positivas o negativas).

De donde $v$ sería de la forma $v=\alpha w, w \geq 0$. Por lo tanto $|v|=|\alpha| w, \mathrm{y}$ como $w \in\left\langle z_{0}\right\rangle$. Así $v \in\left\langle z_{0}\right\rangle$ y entonces se obtiene que $\lambda=s$. $\square$.

\subsection{Sensibilidad al Riesgo}

Cuando uno lee "Sensibilidad al Riesgo" este ocupa dos palabras que parecieran no tener nada en común: sensibilidad y riesgo. Para comenzar se retomará las definiciones que ofrece la Real Academia de la Lengua Española.

Sensibilidad: Capacidad de respuesta a muy pequeñas excitaciones, estímulos o causas.

Riesgo:Dicho de acometer una empresa o de celebrar un contrato: Sometiéndose a influjo de suerte o evento, sin poder reclamar por la acción de estos.

Como se puede observar de lo anterior al referirnos al término sensibilidad al riesgo, esto expresa una cierta capacidad de respuesta a celebrar un evento sometiéndose a un influjo de suerte, sin poder reclamar por la acción sucedida. 
Lo precedente abre un panorama sobre una cierta actitud que tomarán los individuos cuando se les presente una situación desconocida o en la que tengan que recurrir a la suerte o en la que tengan que tomar una decisión bajo incertidumbre.

Para motivar esto matemáticamente hablando, recordemos a Daniel Bernoulli (8/02/1700 - 17/03/1782) un matemático Suizo que escribió en San Petesburgo en 1738 un artículo en latín titulado "Specimen theoriae novae de mensura sortis" que traducido al español se titularía: "Exposición de una nueva teoría sobre la medida del riesgo".

El propósito de ese artículo era mostrar que dos personas expuestas al mismo escenario (lotería); su forma de evaluar y de responder eran diferentes porque se involucraban sus psicologías. En ese artículo Bernoulli plantea 3 ejemplos; entre los que destaca uno muy famoso conocido como la paradoja de San Petesburgo.

Aunque para nuestros días esta Paradoja podría suponer un hecho relativamente claro, en ese tiempo Pascal y Fermat argumentaban que el valor de la lotería debería ser igual a la esperanza matemática; lo que tenía como resultado que el valor de la lotería era idéntico para todas las personas independientemente de la actitud al riesgo de cada individuo.

Para aclarar este punto, se analizará uno de los ejemplos que planteó Bernoulli sobre un individuo llamado "Sempronius" el cual fue enunciado de la siguiente forma:

Sempronius tiene una riqueza en casa de 4000 ducados(moneda) y en un país lejano posee 8000 ducados los cuales sólo los puede poseer en caso de que los transporte por mar. Sin embargo, la experiencia de esos tiempos decía que uno de cada dos barcos era hundido.

En el lenguaje moderno se puede decir que Sempronius se enfrenta 
a un problema de riesgo en su riqueza. Su riqueza se puede ver como una lotería $\bar{x}$, la cual toma el valor de 4000 con probabilidad $1 / 2$ (si el barco es hundido) ó 12000 con probabilidad 1/2 en el otro caso. Se puede denotar la lotería $\bar{x}$ como $(4000,1 / 2 ; 12000,1 / 2)$, como el caso que se plantea es discreto entonces la esperanza matemática esta dada por:

$$
E(\bar{x})=1 / 2(4000)+1 / 2(12000)=8000 \text { ducados }
$$

Pero ahora viene la idea brillante de Sempronius que en vez de poner sus 8000 ducados en una sola nave, el pone porciones de su riqueza en dos naves. Si se asume que los barcos siguen rutas independientes e igualmente peligrosas entonces ahora Sempronius tiene una nueva lotería $\bar{y}$ que esta distribuida de la siguiente manera (4000, 1/4; 8000, 1/2, 12000, 1/4). Como se puede observar si los dos barcos son hundidos entonces Sempronius recibe 4000 ducados, más aún como se supuso que los dos barcos son independientes entonces la probabilidad de que sucedan los dos eventos juntos es la multiplicación de las probabilidades es decir $(1 / 2)^{2}$. Similarmente que los barcos puedan cruzar sucedería con probabilidad $(1 / 4)$ con lo que la recompensa final sería de 12000 ducados. Finalmente también se tiene la posibilidad que sólo uno de los barcos cruce y en tal caso sólo se obtendría la mitad de la recompensa. Con lo que la riqueza final de Sempronius sería de 8000 ducados. Esto conduce a una situación muy interesante, aunque el sentido común indicaría que diversificar los envíos da un mejor resultado. Sin embargo, sí sólo se toma la esperanza matemática entonces Sempronius obtiene:

$$
E(\bar{y})=(1 / 4) * 4000+(1 / 2) * 8000+(1 / 4) * 12000=8000 \text { ducados }
$$

y como se puede observar es el mismo valor que se tenía para $E(\bar{x})$, lo que nos dice que Sempronius es indiferente a la diversificación de los bienes. (El ejemplo anterior es una modificación al artículo original de Bernoulli, 
pero que sigue la misma idea de diversificar y obtener la misma esperanza matemática).

Lo anterior muestra que medir respecto a la esperanza matemática no es suficiente para el objetivo de clasificar cuando la acción de un individuo es más riesgosa que otra.

Una de las cosas más interesantes que planteó Bernoulli en su artículo es que propone una idea de como decir que la alternativa $\bar{y}$ es mejor que la alternativa $\bar{x}$, en donde explica que la mejor forma de que la lotería sea evaluada, es medir con respecto a la esperanza de la utilidad que esta produce. Es decir en vez de tomar la esperanza de la recompensa monetaria, se tomará la esperanza de la utilidad de la riqueza. Lo más interesante de lo que plantea Bernoulli es que nos indica que existe una relación no lineal entre la riqueza y la utilidad que produce consumir la riqueza.

Aunque lo que si es importante marcar es que el individuo necesita medir en algún sentido el grado de satisfacción que obtiene de la recompensa monetaria y es en este sentido que la relación anterior se ve caracterizada por la función de utilidad.

Para ahondar en este tema obsérvese lo siguiente. Sea $u$ que denota la función de utilidad de un individuo, entonces para cada valor de la riqueza se tiene que $u(x)$ denota el nivel de satisfacción o de utilidad que se obtiene vía la riqueza $x$. Aunque el valor de la riqueza es objetivo la transformación por medio de $u(x)$ se vuelve subjetiva y específica para cada individuo dependiendo de su psicología.

Es en este punto, es en donde se hacen los supuestos matemáticos 
sobre la función $u$ los cuales se toman como verdaderos y a partir de aquí estos serán los que nos llevarán por todo lo que se sigue.

Antes de hablar sobre la función $u$ es necesario resaltar la axiomatización que propuso Von Neumman y Morgestern(VN-M) [17], sobre la racionalidad de los individuos, ya que justo esto dió como resultado el teorema sobre la función de utilidad.

Los 4 axiomas que imponen VN-M sobre la racionalidad de los individuos son: completez, transitividad, continuidad e independencia. Los cuales se enuncian de la siguiente manera:

Axioma 1 (Completez) Para cualquier lotería $L, M$, se sigue sólo uno de los siguientes:

$L \prec M, M \prec L$, o $L=M$ (lo anterior se puede leer como $L$ es preferida a $M$ ó $M$ es preferida o no hay una preferencia).

Axioma 2 (Transitividad) Si $L \preceq M$ y $M \preceq N$ entonces $L \preceq N$, esto en palabras indica que la preferencia es consistente respecto a las cadenas. Axioma 3 (Continuidad) Si $L \preceq M \preceq N$ entonces existe una probabilidad $p \in[0,1]$ tal que $p L+(1-p) N=M$. Lo que nos dice es que hay un punto de inflexión entre la mejor y la peor opción.

Otra posibilidad que también fue planteada es sustituir este axioma por: Axioma 3' (Propiedad Arquimediana) Si $L \preceq M \preceq N$, entonces existe una probabilidad $\epsilon \in(0,1)$ tal que $(1-\epsilon) L+\epsilon N \prec M \prec \epsilon L+(1-\epsilon) N$. Este axioma dice que bajo pequeñas desviaciones en las probabilidades las preferencias pueden son mantenidas.

Axioma 4 (Independencia) Si $L \prec M$ entonces para $N$ y $p \in(0,1]$, $p L+(1-P) N \prec p M+(1-p) N$. Este axioma nos plantea que la preferencia es independiente de la posibilidad de recibir otro resultado. 
Una vez, que se conoce lo anterior se puede pasar a un resultado de suma importancia.

Para cualquier individuo (agente) racional (para el cual se satisface 1-4), existe una función $u$ que asigna a cada riqueza $x$ un número real $u(x)$ el cual explica que para cualquiera dos loterías $L, M$; se dice que $L \prec M$ si y sólo si $E(u(L))<E(u(M))$, en donde $E(u(L))$ denota el valor esperado de $u$ en $L$.

Para fijar ideas, obsérvese que sucede en el ejemplo de Sempronius si se utiliza $u(x)=\sqrt{x}$, la cual es una función creciente y cóncava.

$E(u(\bar{x}))=1 / 2(\sqrt{4000})+1 / 2(\sqrt{12000})=86,4$

$E(u(\bar{y}))=1 / 4(\sqrt{4000})+1 / 2(\sqrt{8000})+1 / 4(\sqrt{12000})=87,9$

y entonces como el sentido común indica, Sempronius preferiría dividir su riqueza en más embarcaciones.

Cuando se hizo lo anterior, se usó que Sempronius tomó una actitud en la cual quería evitar lo más posible su riesgo, lo cual conduce a explicar como se clasifican las acciones de un individuo.

\subsubsection{Definición y Caracterización de la Aversión al Riesgo}

Definición 7.4.1. Una lotería es actuarialmente justa cuando su valor esperado es cero; es decir: $p x+(1-p) y=0$ para $p \in(0,1)$

Por ejemplo se pueden considerar los juegos de suma cero.

Un individuo con probabilidad $1 / 2$ gana 10 pesos y con probabilidad $1 / 2$ pierde 10 pesos.

Entonces la esperanza de esté juego: $E(x)=1 / 2(10)+1 / 2(-10)=0$. 
Definición 7.4.2. Un agente se le dice averso al riesgo, si para cualquier nivel de riqueza $w$, no está dispuesto a aceptar cualquier lotería que tiene valor esperado igual a cero.

$\forall w, \forall \bar{z}$ con $E(\bar{z})=0, E(u(w+\bar{z})) \leq u(w)$. En otras palabras se puede decir que un agente que es averso al riesgo prefiere su situación inicial con un nivel de riqueza $w$ que jugar un juego actuarialmente justo en el cual con probabilidad p gana $x$ y con probablilidad $(1-p)$ gana $y$. Más aún lo anterior se puede ver como:

$$
E(u(w+\bar{z})) \leq u(w+E(\bar{z}))
$$

ó

$$
p U(w+x)+(1-p) U(w+y)<U(p(w+x)+(1-p)(w+y))
$$

Otra forma de pensar lo anterior es que el agente prefiere quedarse con lo que el conoce como seguro(cierto) en vez de entrar a la lotería.

Obsérvese que sucede para el caso de Sempronius. Si se considera el ejemplo en el cual solo se puede mandar a traer su riqueza en un barco entonces se tiene que la riqueza inicial es 4000 y que $\bar{z}=0$ ó 8000 con probabilidad $1 / 2$, tomase $u(x)=\sqrt{x}$. Entonces la desigualdad anterior se transforma en:

$1 / 2 u(12000)+1 / 2 u(4000) \leq u(8000)$ en donde $\mathrm{u}(8000)$ sale de tomar los 4000 de la riqueza inicial más la esperanza de la lotería $\bar{z}$ que es 4000 . De donde calculando los valores tenemos que: $86.4<89$. 4 por lo que como se supuso Sempronius es un individuo averso al riesgo. 


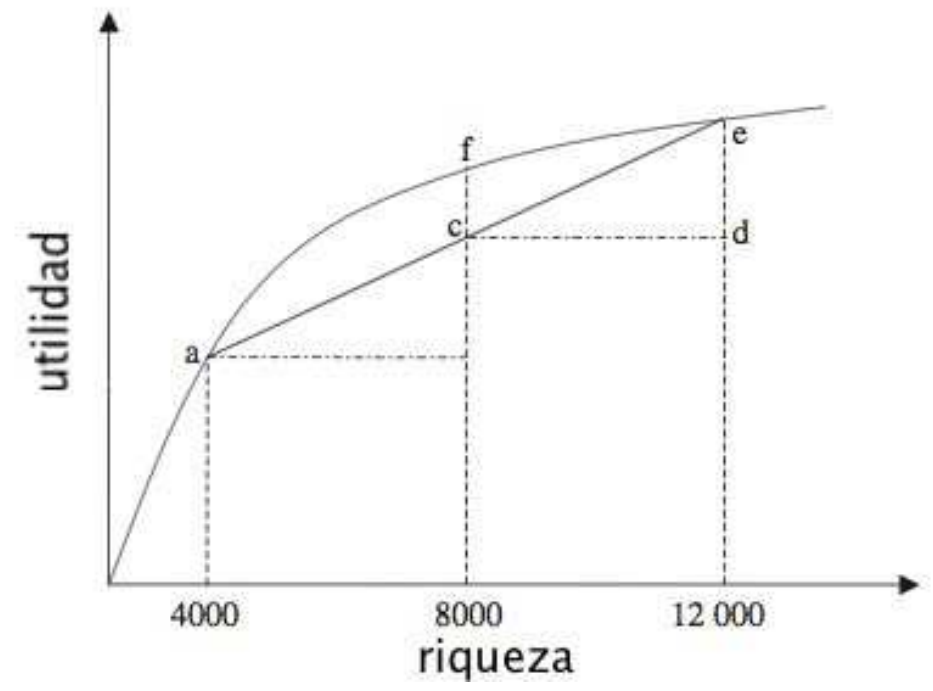

Lo que se puede observar en la imagen anterior es que cuando la función es cóncava se tiene que el punto $\mathbf{f}$ que representa a $u(w+E(\bar{z}))$ es mayor que el punto c el cual representa $E(u(w+\bar{z}))$ para un $\bar{z}$ fijo. Entonces lo que se explica es que cuando una función es cóncava los valores para los cuales el agente tiene una certeza son mayores que los valores que podría recibir respecto a los cuales tiene incertidumbre, dando como resultado que el agente es averso al riesgo. Así mismo también se puede obtener una gráfica análoga para el caso cuando el agente es propenso al riesgo.

Proposición 7.4.3. Un agente con función de utilidad $u$ es averso al riesgo sí y sólo sí su función de utilidad u es cóncava.

\section{Demostración:}

La prueba sobre la suficiencia de que $u$ debe ser cóncava se basa en utilizar la expansión de la serie de Taylor de segundo orden para $u(w+z)$ alrededor de $(w+E \bar{z})$. Para cualquier $\bar{z}$ se tiene que:

$u(w+z)=u(w+E(\bar{z}))+(z-E(\bar{z})) u^{\prime}(w+(E \bar{z}))+1 / 2(z-E(\bar{z}))^{2} u^{\prime \prime}(\xi(z))$ para algún $\xi(z) \in[z, E(\bar{z})]$. Como esto se debe cumplir para todo $\bar{z}$, se sigue que tomando la esperanza con respecto a $u(w+\bar{z})$ se tiene: $E(u(w+\bar{z}))=u(w+E(\bar{z}))+u^{\prime}(w+E(\bar{z})) E(\bar{z}-E(\bar{z}))+1 / 2 E[(\bar{z}-$ 
$\left.E(\bar{z}))^{2} u^{\prime \prime}(\xi(z))\right]$

usando las propiedades de la esperanza se nota que el segundo término es igual a 0 , ya que $E(\bar{z}-E(\bar{z}))=E(\bar{z})-E(\bar{z})=0$. Más aún, si $u$ es uniformemente negativa entonces el tercer término del lado derecho se puede ver como la esperanza de una variable aleatoria $(\bar{z}-E(\bar{z}))^{2} u^{\prime \prime}(\xi(\bar{z}))$ que dado lo anterior siempre es negativo ya que es el producto de un número elevado al cuadrado por uno negativo; de donde se tiene que la suma de los tres términos es menor que $u(w+E(\bar{z}))$. Con lo que se prueba la suficiencia. Ahora la parte de ser necesario se hará por contradicción. Supóngase que $u$ no es cóncava, entonces debe existir un $w$ y un $\delta>0$ para el cual $u^{\prime \prime}(x)$ es positiva en el intervalo $[w-\delta, w+\delta]$, ahora si se toma un pequeño riesgo $\bar{\epsilon}$ con media cero y sea de tal forma que $w+\bar{\epsilon}$ se quede totalmente contenido en $(w-\delta, w+\delta)$; entonces usando la expansión en serie de Taylor de segundo orden se tiene:

$E(u(w+\bar{\epsilon}))=u(w)+1 / 2 E\left[\epsilon^{2} u^{\prime \prime}(\xi(\epsilon))\right]$. Como el soporte de $\xi(\bar{\epsilon})$ esta contenido en $[w-\delta, w+\delta]$ en donde $u$ es localmente convexa entonces $u^{\prime \prime}(\xi(\bar{\epsilon}))$ es positiva para todas las realizaciones de $\bar{\epsilon}$ entonces se sigue que $E\left[\bar{\epsilon}^{2} u^{\prime \prime}(\xi(\bar{\epsilon}))\right]$ es positiva y entonces se tiene que $E(u(w+\bar{\epsilon}))>u(w)$. De donde por tomar la lotería $\bar{\epsilon}$ se tiene que el agente no es averso al riesgo. Contradicción.

Definición 7.4.4. Un agente se dice que es neutral al riesgo, cuando es indiferente a aceptar cualquier juego actuarialmente justo. Esto se puede ver respecto a la función de utilidad como:

$$
E(u(w+\bar{z}))=u(w+E(\bar{z}))
$$

$o ́$

$$
p U(w+x)+(1-p) U(w+y)=U(p(w+x)+(1-p)(w+y))
$$


de donde se puede notar de manera inmediata que una función que es lineal cumple lo anterior.

Definición 7.4.5. Un agente se dice que es amante(propenso) al riesgo cuando está dispuesto a aceptar cualquier juego actuarialmente justo. Lo cual se puede observar como:

$$
u(w+E(\bar{z})) \leq E(u(w+\bar{z}))
$$

ó

$$
U(p(w+x)+(1-p)(w+y))<p U(w+x)+(1-p) U(w+y)
$$

de manera similar con lo hecho respecto a la aversión al riesgo se tiene que un agente es amante al riesgo si y solo si su función de utilidad u es

convexa. Lo cual dice que el agente siempre está dispuesto a arriesgarse con respecto a lo incierto más que buscar asegurar lo que el conoce como seguro(cierto).

La demostración de lo anterior se sigue de manera análoga al caso en el que se tiene aversión al riesgo.

Una vez dado lo anterior se esta en condiciones sobre como son las funciones respecto al comportamiento de los agentes, ahora el siguiente paso es observar que sucede si tomamos dos agente, la pregunta que surge es: ¿será posible de algún modo compararlos?; es decir si se puede determinar en algún sentido cuando un agente tiene un comportamiento con más aversión al riesgo.

\subsubsection{Medidas de Aversión}

Una forma de medir el grado de aversión al riesgo es preguntarle al agente ¿cuánto está dispuesto a invertir para eliminar un riesgo $(\bar{z})$ con 
media cero?. Para contestar a la pregunta anterior se tendrá que definir un nuevo concepto al cual se le conocerá como el riesgo premium $\Pi$ asociado al riesgo $(\bar{z})$. Lo que explica este concepto es que un agente con función de utilidad $u$ y con riqueza inicial $w$, su riesgo premium es de tal forma que se satisface:

$$
E(u(w+\bar{z}))=u(w-\Pi)
$$

Lo cual significa que el agente termina con el mismo bienestar ya sea aceptando el riesgo o pagando el riesgo premium. Una notación convencional para este concepto es que para cuando $\bar{z}$ tiene una esperanza distinta de cero entonces se le conoce con el nombre de certeza equivalente. Lo cual nos indica que la "Certeza Equivalente" $e$ de un riesgo $\bar{z}$ es el incremento que se tiene seguro en la riqueza con el cual se obtiene el mismo efecto que tomar el riesgo $\bar{z}$. Lo anterior en fórmula se expresa como:

$$
E(u(w+\bar{z}))=u(w+e)
$$

Como se puede observar cuando $\bar{z}$ tiene media cero comparando 7.7 y 7.8 implica que la certeza equivalente $e$ de $\bar{z}$ es igual a menos su riesgo premium $\Pi$.

Una cosa que se puede notar es que el riesgo premium es positivo cuando el agente es averso al riesgo, cero cuando es neutral al riesgo y es negativo en el caso cuando el agente es propenso o amante al riesgo. Un hecho relevante a notar es que el riesgo premium es medido en las unidades de riqueza, lo que nos indica el grado de afectación vía la riqueza y no el grado de bienestar.

Un detalle a resaltar es que como se puede ver el riesgo premium depende tanto de la distribución inicial de la riqueza $w$, el riesgo $\bar{z}$ y de la función de utilidad $u$, por lo que en un sentido es mejor estimar lo que el agente 
está dispuesto a pagar para eliminar el riesgo de media cero considerando pequeños riesgos.

Así que se puede asumir que la $\mathrm{E}(\bar{z})=0$, si se supone que las funciones que se están usando son de clase $\mathbb{C}^{2}$ entonces desarrollando la expansión en serie de Taylor de segundo orden respecto a 7.7 del lado izquierdo y de primer orden para el lado derecho se obtiene que:

$$
\begin{aligned}
& u(w-\Pi) \simeq u(w)-\Pi u^{\prime}(w) \\
& E(u(w+\bar{z})) \simeq E\left[u(w)+\bar{z} u^{\prime}(w)+1 / 2 \bar{z}^{2} u^{\prime \prime}(w)\right. \\
&=u(w)+u^{\prime}(w) E(\bar{z})+1 / 2 u^{\prime \prime}(w) E\left(\bar{z}^{2}\right) \\
&=u(w)+1 / 2 \sigma^{2} u^{\prime \prime}(w)
\end{aligned}
$$

donde $E(\bar{z})=0$ y $\sigma^{2}=E\left(\bar{z}^{2}\right)$ que de hecho denota la varianza del resultado de la lotería. Entonces usando las aproximaciones anteriores para 7.7 se tiene que:

$$
\Pi \simeq 1 / 2 \sigma^{2} A(w)
$$

en donde la función $A$ se define de la siguiente forma: $A(w)=-\frac{u^{\prime \prime}(w)}{u^{\prime}(w)}$ la cual es positiva en el caso de aversión al riesgo, cero en el caso en el que hay neutralidad al riesgo y negativa para el caso del amante al riesgo.

La función $A(\cdot)$ es conocida como la medida de aversión absoluta al riesgo del agente, de la ecuación (7.9) se deduce que el riesgo premium es igual a la mitad de la varianza por la medida de aversión absoluta al riesgo. La ecuación 7.9 se conoce como la aproximación de Arrow-Pratt, la cual fue desarrollada independientemente por Arrow (1963) y por Pratt en (1964). Un hecho a resaltar es que bajo esta medida dos individuos tienen la 
misma aversión al riesgo si estos dos individuos están dispuestos a arriesgar la misma cantidad monetaria en términos absolutos, pero el problema surge debido a que depende de la renta(proporción que se arriesga) del individuo.

La medida de aversión absoluta al riesgo(MAAR) es la proporción de decaimiento de la utilidad marginal. En particular (MAAR) es la tasa por la cual la utilidad marginal decrece cuando la riqueza se incrementa en una unidad. Un detalle a resaltar es que (MAAR) es dependiente de las unidades que se utilizan por lo que los economistas no quedaron conformes y entonces un detalle que se les ocurrió fue definir el índice relativo de aversión al riesgo $R$, como la tasa para la cual la utilidad marginal decrece cuando la riqueza se incrementa en un por ciento. En términos de la teoría económica esta medida es simplemente la elasticidad de la riqueza respecto de la utilidad marginal, la cual se expresa de la siguiente manera:

$R(w)=-\frac{d u^{\prime}(W) / u^{\prime}(w)}{d w / w}=-\frac{w u^{\prime \prime}(w)}{u^{\prime}(w)}=w A(w)$. Cabe notar que la $d w=$ 1 debido a que se toma la riqueza como una variable $w$ y como se puede dar uno cuenta la nueva medida no es más que el producto de la medida absoluta de aversión al riesgo por la riqueza.

Cabe señalar que estas dos medidas son las que se utilizan de manera más frecuente.

\subsubsection{Funciones Clásicas de Utilidad}

Históricamente durante los años sesentas las funciones de utilidad que se utilizaban eran funciones cuadráticas de la forma:

$$
u(w)=a w-1 / 2 w^{2}
$$

para $w \leq a$. Nótese que el dominio de la riqueza en el cual $u$ se define, viene precedido de buscar que la función sea no decreciente y eso sólo es cierto en el caso de que w sea más pequeño que a. Algo relevante de este 
tipo de funciones es que era suficiente tener los primeros dos momentos así que realidad son funciones que dependen de la media y la varianza de las loterías.

Cabe señalar que este tipo de funciones exhiben un riesgo absoluto creciente de aversión al riesgo. Lo cual se concluye por el siguiente hecho:

$$
A(w)=\frac{1}{a-w} \Rightarrow A^{\prime}(w)=\frac{1}{(a-w)^{2}} .
$$

El segundo conjunto de funciones clásicas son las conocidas como: funciones con coeficiente absoluta de aversión al riesgo constante (CARA) por sus siglas en inglés, las cuales se representan por:

$$
u(w)=-\frac{\mathrm{e}^{-\lambda w}}{\lambda}
$$

donde $\lambda$ es un escalar positivo. Algo que es importante notar que para este tipo de funciones el dominio son todos los $\Re$; un hecho sustancial es que exhiben un coeficiente de aversión al riesgo absoluto constante es decir

$$
A(w)=\lambda
$$

para todo w. Un hecho significativo es que la aproximación que fue hecha por Arrow- Pratt para el caso en el que u es exponencial y $\bar{w}$ se distribuye normal con media $\mu$ y varianza $\sigma^{2}$ es exacta; Obsérvese que si se toma la esperanza sobre $u(\bar{w})$ para este caso particular se tiene que:

$$
\begin{aligned}
E(u(\bar{w})) & =-\frac{1}{\sigma \lambda \sqrt{2 \pi}} \int \exp (-\lambda w) \exp \left(-\frac{(w-\mu)^{2}}{2 \sigma^{2}}\right) d w \\
& =-\frac{1}{\lambda} \exp \left(-\lambda\left(\mu-\frac{1}{2} \sigma^{2}\right)\right)\left[\frac{1}{\sigma \sqrt{2 \pi}} \int \exp \left(-\frac{\left(w-\left(\mu-\frac{1}{2} \lambda \sigma^{2}\right)\right)^{2}}{2 \sigma^{2}}\right) d w\right] \\
& =-\frac{1}{\lambda} \exp \left(-\lambda\left(\mu-\frac{1}{2} \lambda \sigma^{2}\right)\right)=u\left(\mu-\frac{1}{2} \lambda \sigma^{2}\right) .
\end{aligned}
$$

La última igualdad se sigue de tomar la completación de una $N(\mu-$ $\left.\frac{1}{2} \lambda \sigma^{2}, \sigma\right)$ la cual integra 1 . Más aún el riesgo premium para este caso toma 
la forma de $\frac{1}{2} \sigma^{2} A(w)$ Una de las grandes discusiones sobre el uso de las funciones CARA es que el coeficiente absoluto de aversión al riesgo sea constante y no decreciente como algunos de los economistas lo esperan.

El tercer gran grupo de funciones que ha sido trabajado por los investigadores son las funciones con utilidad potencial es decir:

$$
u(w)=\frac{w^{1-\gamma}}{1-\gamma}
$$

donde $\gamma$ es un escalar positivo y diferente de 1. Este tipo de funciones son recurrentes en la literatura debido a que el coeficiente relativo de aversión al riesgo $R(w)=\gamma$ para todo $\mathrm{w}$, debido a que:

$$
A(w)=\gamma / w
$$

Más aún, este tipo de funciones presenta un comportamiento decreciente en el coeficiente de aversión al riesgo absoluto y contante para el relativo. En particular a este grupo de funciones se les conoce como funciones de constantes relativas de aversión al riesgo (CRRA) por sus siglas en inglés. Como se puede observar existe un detalle para el caso cuando $\gamma$ es igual a 1. De donde su generalización viene dada de la siguiente forma:

$$
u(w):=\left\{\begin{array}{lc}
\frac{w^{1-\gamma}}{1-\gamma} & \text { si } \gamma \geq 0, \gamma \neq 1 \\
\ln (w) & \text { si } \gamma=0 .
\end{array}\right.
$$

La ventaja que ofrece utilizar este tipo de funciones es que el riesgo relativo premium es independiente de la riqueza. Por lo cual algunos autores las prefieren.

Lo anterior fue una breve introducción sobre la manera de medir el riesgo, lo cual abre todo un panorama sobre el significado de sensibilidad al riesgo y las formas en que se mide. 
Antes de cerrar este rápido paseo por la funciones de utilidad se mencionarán otros grupos de funciones de funciones típicas que se han utilizado a lo largo de los años

Las funciones de utilidad lineales fueron de las primeras en ser utilizadas, un ejemplo de su recurrencia es el caso en el que se tienen bienes de sustitutos perfectos (Un bien es un sustituto perfecto de otro, solamente si puede ser usado exactamente de la misma forma y con el mismo resultado y entonces es cuando un consumidor no tiene ningún incentivo para preferir un bien sobre el otro.)

Las funciones de utilidad lineales se representan de la siguiente forma:

$$
u(x)=a+b x,
$$

donde $a, b \in \mathbb{R}$.

Otro grupo muy recurrente son las funciones de utilidad logarítmica las cuales son regularmente utilizadas debido a que son un ejemplo de funciones DARA (coefiente absoluto de aversión al riesgo decreciente); aunque uno de sus mayores usos se da en la formación de portafolios. Se caracterizan por:

$$
u(x)=b \ln (x+c),
$$

donde $b>0$ y $c \geq 0$ 


\section{Capítulo 8}

\section{Glosario}

Notación sobre el uso de la letra "P".

- Cadenas de Markov.

$P_{i j}>0$ denota la probabilidad de que al siguiente estado la cadena haya pasado del estado i al estado j. $P_{i j}>0$.

$P_{i j}(n)>0$ denota la probabilidad de ir de i a j exactamente en $\mathrm{n}$ pasos.

$P^{n}$ denota la potencia n-ésima de la matriz P.

- Propiedad Producto.

$P_{i}$ denota la i-ésima fila de la matriz $\mathrm{P}$.

- Reordenación en base a Cadenas $P^{C}$ denota la restricción de $\mathrm{P}$ a la clase $\mathrm{C}$.

- Recursión $P^{n}$ denota la potencia n-ésima de la matriz $\mathrm{P}$.

- Proceso iterativo $\mathrm{P}(\mathrm{m})$ denota la m-ésima iteración. 


\section{Bibliografía}

[1] Arriaga, S. Problemas de Control de Markov con Recompensa Total Esperada en Espacio Finitos: Casos Neutral y Sensible al Riesgo, Tesis de Maestría en Matemáticas, UAM-Iztapalapa, 2008.

[2] Ash, R. Probability and Measure Theory. Academic Press, India, 2008.

[3] Caballero, M.E. Riverdo, V.M. Uribe, G. Velarde,C. Cadenas de Markov. Un Enfoque Elemental. SMM 2004.

[4] Cavazos-Cadena R. and Montes de Oca R., Optimal stationary policies in controlled Markov chains with expected total-reward criterion. Reporte de Investigación. No. 04.0405.I.01.010.99, UAM-Iztapalapa, 1999.

[5] Cavazos-Cadena R. and Montes de Oca R., Optimal stationary policies in risk-sensitive dynamic programs with finite state space and nonnegative rewards. Applicationes Mathematicae, Warsaw, Vol. 27, 2, 167-185, 2000.

[6] Cavazos-Cadena R. and Montes de Oca R., Stationary optimal policies in a class of multichain positive dynamic programs with finite state space and risk-sensitive criterion. Applicationes Mathematicae, Warsaw, Vol. 28, 93-109, 2001.

[7] Dunford, N. and Schwartz, J.T. Linear Operators, Part I. Intersciences, New York, 1958. 
[8] Eeckhoudt, L. Gollier, C. Schlesinger, H. Economic and Financial Decision under Risk. Princeston University Press, 2005.

[9] Feinberg, E. and Schwartz, A. A Handbook of Markov Decision Processes: Methods and Applications. Kluwer, 2001.

[10] Gantmacher, F.R., The Theory of Matrices, Vol. II. Chelsea, New York, 1959.

[11] Grone, R., Johnson, C., Sá. M, Wolkowicz. H. Positive definite completions of partial Hermitian matrices, Linear Algebra and its Applications, Vol. 58, 109-124, 1984.

[12] Howard, R. and Matheson, J.E. Risk-sensitive Markov decision processes. Management Sciences, Vol.18 no. 7, 356-369, 1972.

[13] Hu, Q. and Yue, W. Markov Decision Processes with their Applications. Springer, 2008.

[14] Bondarenko, I. Groups Generated by Bounded Automata and their Schreiergraphs. PHD Thesis, 2007.

[15] Jordan, C. Traité des Substitutions et des Équations Algébriques, 2da. ed., Gauthier-Villars y A. Blanchard, Paris, 1957.

[16] Kemeny, J.G. and Snell, L. G. Finite Markov Chains. Van Nostrand, Princeton, New Jersey, 1960.

[17] Kreps, D. M. A Course on Microeconomics Theory. McGraw Hill, 1995.

[18] Lancaster, P. and Tismenetsky, M. The Theory of Matrices with Applications. Academic Press, 1985.

[19] Meyer, D. C. Matrix Analysis and Applied Linear Algebra Book and Solution Manual. SIAM, 2000,

[20] Pease, M. C. Methods of Matrix Algebra. Academic Press, 1965. 
[21] Ross, S. Introduction to Stochastic Dynamic Programmming. Academic Press, 1983.

[22] Rothblum, U.G. Algebraic eigenspaces of nonnegative matrices. Linear Algebra and its Applications, Vol.12, 281-292, 1975.

[23] Seneta, E. Non-negative Matrices and Markov Chains. Springer, 2006.

[24] Sladký K. and Montes-de-Oca R., Risk-sensitive average optimality in Markov decision chains. Operations Research Proceedings 2007. Eds. J. Kalcsics and S. Níkel, 69-74, Springer, 2008.

[25] Sladký K. Bounds on discrete dynamic programming recursions I. Models with non negative matrices. Kybernetika, Vol. 16, No. 6, 526$547,1980$.

[26] Sladký K. On the set of optimal controls for Markov chains with rewards. Kybernetika, Vol. 10, No. 4, 350-367, 1974.

[27] Sladký K. On the existence of stationary optimal policies in discrete dynamic programming. Kybernetika, Vol. 17, No. 6, 489-513, 1981.

[28] Takayama, A. Mathematical Economics. Cambridge University Press, 1985.

[29] Zijm, W.H.M. Asymptotic behaviour of the utility vector in a dynamic programming model. Memorandum COSOR 80-04, Eindhoven University of Technology, Eindhoven, 1980.

[30] Zijm, W.H.M. Geometric convergence in average reward Markov decision processes. Memorandum COSOR 80-08, Eindhoven University of Technology, Eindhoven, 1980.

[31] Zijm W.H.M. Nonnegative Matrices in Dynamic Programming. Amsterdam, Mathematisch Centrum, 1983. 


\section{REDUCIBILIDAD EN MATRICES FINITAS NO NEGATIVAS CON APLICACIONES A ECONOMÍA Y CONTROL ESTOCÁSTICO}

ASESOR: DR. JOSÉ RAÚL MONTES DE OCA MACHORRO

JURADO CALIFICADOR:

PRESIDENTE: DR. JUAN GONZÁLEZ HERNÁNDEZ UNAM

SECRETARIO: DR. JULIO CÉSAR GARCÍA CORTE UAM-I

VOCAL:

\author{
TESIS QUE PRESENTA: \\ VÍCTOR MANUEL MARTÍNEZ CORTÉS \\ PARA OBTENER EL GRADO DE \\ MAESTRO EN CIENCIAS \\ MATEMÁTICAS APLICADAS E INDUSTRIALES
}

